Thesis
UC-28

Issued: December 1977

\title{
Investigation of Multi-Charged Heavy Ion \\ Production in an Electron Beam Ion Source
}

Robert Wray Hamm

MASTII

\author{
Robert Wray Hamm
}

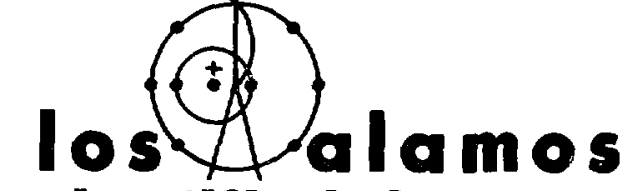

scientific laboratory

of the University of California

LOS ALAMOS. NEW MEXICO 87545

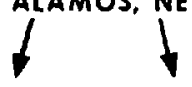

An Affirmative Action/Equal Opportunity Employer 
This thesis was accepted by the Physics Department, Texas A\&M University, College Station, Texas 77843, in partial fulfillment of the requirements for the degree of Doctoi of Philosophy. It is the independent work of the author and has not been edited by the Technical Information staff.

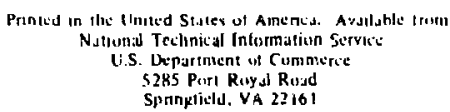

\begin{tabular}{|c|c|c|c|c|c|c|c|c|c|}
\hline Hererestiche & 53.611 & 126.150 & 7.25 & $251-275$ & 10.75 & $376-4011$ & {$[3.40$} & 501.525 & 15.25 \\
\hline 1001-kISS & 400 & $151-175$ & 8.311 & $276-301$ & 11.00 & $401-425$ & 13.25 & $\$ 26.55 t 1$ & 15.50 \\
\hline $1136 \cdot 050$ & 4.50 & i 76.2004 & 9.00 & $301.3 \geq 5$ & 11.75 & $426-450$ & 14.011 & $551-575$ & 16.25 \\
\hline IISI.4175 & 5.25 & $201-225$ & 9.25 & $326-350$ & 12.01 & $451-475$ & 14.50 & $576-600$ & 16.50 \\
\hline $1176 \cdot 100$ & 6.00 & 226.250 & 9.50 & 351.375 & 12.50 & 476.5011 & IS.III & G011-up & --1 \\
\hline
\end{tabular}

1. Add 32.sit for each additional 100-page increment from 601 pages up,

This report was prepured os an arcount of wiork sponsared by the United Stakes Lovernment Nether the United Stales nor the United Seates Department of Einergy, nor any of their employers, nor anv of their contrartiors. subcontractors, ar their cmployees. makes ank wartuniv, express ur limnlisd. dssunies uny legal liabulty or responsibility fiur the a. ru rac $v$ completeness, or unefutness of eny informatisis appatatus nat infringe printely owned nghts. 


\section{INVESTIGATION OF MULTI-CHARGED HEAVY ION PRODUCTION IN AN ELECTRON BEAM ION SOURCE}

A Dissertation

by

ROBERT WRAY HAMM

Submitted to the Graduate college of

Texas A\&M University

in partial fulfillment of the requirement for the degree of

DOCTOR OF PHILOSOPHY

December 1977

Major Subject: Physics

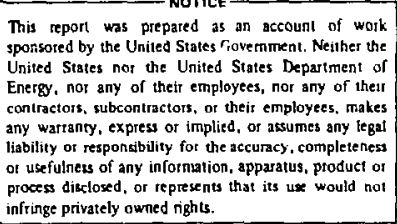


INVESTIGATION OF NULTI-CHARGED HEAVY ION PRODUCTION IN AN

ELECTRON BEAM ION SOURCE

A Dissertation

by

ROBERT TRAY HAMM

Approved as to style and content by:

$\underset{\text { Pant A Tenfeste }}{\text { (Chairman of committee) }}$

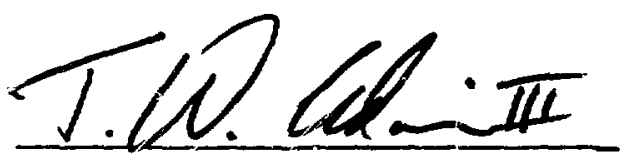

(Head of Department)
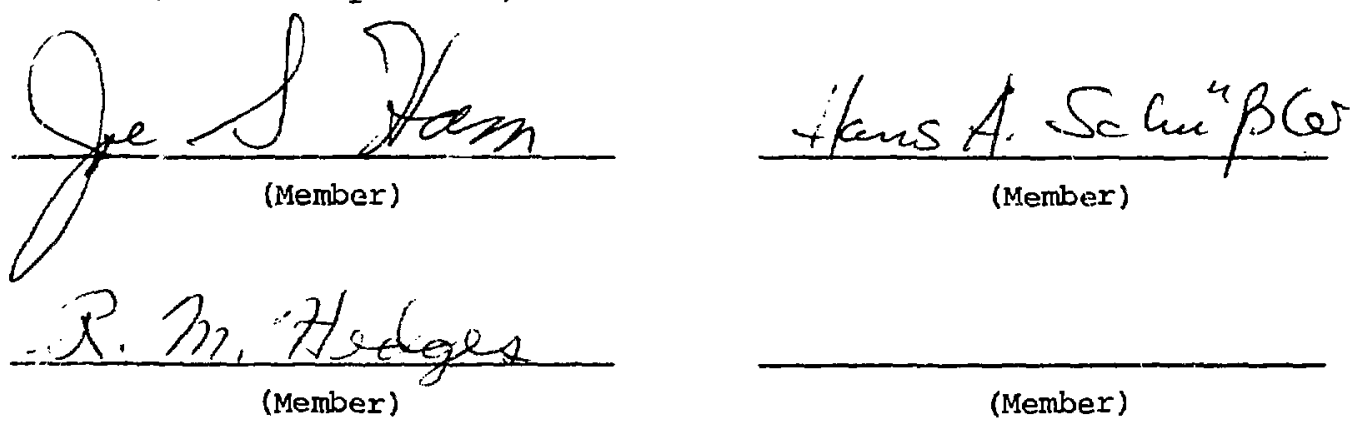

(Member)

(Member)

December 1977

Iv 
I INTRODUCTION . . . . . . . . . . . . . . . . . . 1

A. History of Heavy Ion Accelerators . . . . . . . . 1

B. Uses of Heavy Ions . . . . . . . . . . . . 3

C. Convential Ion Sources ............. 4

D. New Ion Source Concepts.............. . 4

E. Electron Beam Ion Source . . . . . . . . . . 6

II THEORETICAL DESCRIPTION . . . . . . . . . . . . 11

A. Operating Frinciples . . . . . . . . . . . 11

E. Ionization Theory . . . . . . . . . . . . 13

C. Vacuum Considerations.............. . . 22

D. Electron Beam Focussing.............. 23

E. Ion Containment Dynamics . . . . . . . . . 28

III EXPLRIMFITAL APPARATUS............... 40

A. General Description. . . . . . . . . . . . . 40

B. Vacuum system . . . . . . . . . . . . 42

C. Electron Beam System .............. 48

D. Trapping and Pulsing System . . . . . . . 57

E. inalysis system............... . . 60

IV EXPERIMENTAL MEASUREMENTS ................ 63

A. Vacuum Results ... . . . . . . . . . . 63

B. Electron Beam :heasurenents . . . . . . . . 67

C. Ion Production Results . . . . . . . . . 71

$\mathrm{V} \quad$ DISCUSSION OF RESULTS AND CONCLUSIONS . . . . . . 86

ACKNOWLEDCEMENTS . . . . . . . . . . . . . . . . . 91

REFERENCES . . . . . . . . . . . . . . . . . . . . . 92 
Investigation of Multi-Charged Heavy Ion Froduction in an Electron Beam Ion Source. ( December, 1977) Sobert Wray Ham, B. S., University of Southweatern Louisiana; M. S., Florida State University Chairman of Advisory Committee: Dr. Robert A. Keisefirk Measurements of multi-charged heavy ions produced in an Electrin Beam Ion Source (EBIS) have been carried out with a test model ion source $20 \mathrm{~cm}$ in length. This test modei utilized an electron gun placed external to the bore of the focussing solenoid in order to achieve electrostatically focussed electrori beams and isolation of the vacuum surrounding the electron gun from the vacuum in the ionization region within the solenoid bore, An ultrahigh vacuum system utilizing liquid nitrogen $\left(77^{\circ} \mathrm{K}\right)$ cryopumping was used to achieve the low pressures needed in th ionization region for the operation of this ion source.

Several technical froblems limited the operation of this test model and prevented a thorough investigation of the ionization processes in the ion source, but the experimental results have shown qualitative agreement with the theoretical calculations for the operation of this type of ion source. Even with the problems of an insufficient vacuum and electron beam focussing field, measurable currents of $\mathrm{C}^{+5}$ and $\mathrm{A}^{+8}$ ions have been produced. The present experimental results suggest that the approach taken in this work of using an external electron gun and cryopumping in the EBIS to achieve the large electron beam current density and low vacuum necessary for successful operation is a viable one. Such an ion source can be used to create highly-charged heavy ions for injection into a cyclotron or other type of particle accelerator. 
In recent years, the use of heavy ion beams in scientific research has greatly increased. This growing use of heavy elements as accelerated projectiles has stimulated study of the ion sources used to produce them. The expanding requirements for ion sources for the large new heavy ion accelerators currently in operation or under construction have provided the impetus for the present study on the development and performance of a new type of ion source.

In the historical review and introduction which follows, the need for new types of ion sources for highly-charged heavy ions is illustrated and the Electron Beam Ion Source (EBIS) is shown to be a solution for this need. Chapter II presents the theory of the EBIS and its operation. A description of the apparäcus designed and constructed at Texas A\&M University is presented in Chapter III, and the preliminary results obtained from this device are given in chapter IV. Interpretation of these results, and a summary of their implications is presented in the final chapter.

\section{A. History of Heavy I n Accelerators}

The formation and acceleration of ions heavier than helium occurred only a short time after the appearance of particle accelerators. Sloan and Lawrence (1931) constructed a linear accelerating device for mercury ions as the first heavy ion machine, and Alvarez (1940) next demonsirated the acceleration of carbon ions in the 37-inch cyclotron at Berkeley. Neither accelerator produced beams useful for nuclear studies, and it was some years later before heavy ions became useful as tools in nuclear fhysics. iruclear reactions induced by heavy ions were first detected by Miller et al. (1950) using carbon ions accelerated in the 60-inch cyclotror: at Berkele'. The low beam intensity ( $10^{5}$ particles per second) ans large energy spread made these beams unsuitable for most experimental

The citations in this dissertation follow the style of the Reviews of Modern Physics. 
work, but they were sufficient for the production of transuranic elemerts (Ghiorso et al., 1951). Later, a paper by Breit, Hull, and Gluckstern (1952) pointed out many of the characteristics of heavy ion reactions and suggested several types of experiments, which then led to further development of heavy ion production and acceleration.

In the sarly work at Berkeley on carbon ion acceleration, $\mathrm{C}^{+2}$ ions were extracted from the ion source within the cyclotron and accelerated on the third harmon $\approx$ of the deuteron acceleration frequency. Since some of the accelerating particles were stripped to $\mathrm{C}^{+6}$ in the residual vacuum of the cyclotron while near the center, it was then possible for these fully stripped ions to be accelerated on the first acceleration harmonic for the deuteron (Miller, 1952). This method was also used for carbon ion acceleration at Birmingham (walker and Fremlin, 1953), Stockholm (Atterling, 1954), and Saclay (Debraine, 1955).

Since this essentially stochastic method provided heavy ion beams of very low intensity and poor energy resolution, work was begun on the design and constxuction of cyclotrons to accelerate a specific charge state of ions extracted from the source. In 1953, motivated by weapons research, Livingston (1954) and co-workers at Oak Ridge accelerated intense beams of $\mathrm{N}^{+3}$ ions directly from an ion source in a specially built cyclotron. In 1957, $\mathrm{N}^{+3}$ ions were also extracted from an ior, source and accelerated in the 120-cm cyclotron at Leningrad (Alkhazov et al., 1956). A laxge number of accelerators either constructed or modified to accelerate heavy ions then followed-very quickly. These included cyclotrons, Alvarez-type ?inear accelerators, and electrostatic machines such as the tandem Van de Graaffs (Hubbard, 1961).

After this initial work, the development of heavy ion accelerators flourished and the emphasis then turned to the production of heavier ions and higher energies. The appearance of the variable energy isochronous cyclotron presented the potential for acceleration of all heavy ions at variable energies, and this versatile cyclotron, as well as the other types of machines listed above, provided the heavy ion beams used for the experimental work done in the 1960's (Livingston, 1970). During this time, large heavy ion accelerators were proposed and constructed in order to extend the range of elements and the limit of possible enexgies 
(Blann, 1971). Synchrotrons and linacs are now being used for very high energy heavy ions, and many new forms of heavy ion accelerators, including combinations of already existing types, have been proposed (Grunder, $1975,1976)$.

\section{B. Uses of Heavy Ions}

Since the earliest days of heavy ion acceleration, the leading users of heavy ions have been nuclear physicists and nuclear chemists. A great volume of experimental work in nuclear physics and chemistry has been successfully completed in the past 25 years through the use of heavy ions, and this field continues to be important and interesting. The properties which make heavy ion beams useful for the study of nuclear physics and chemistry are their large charge and mass. The large charge produces a strong polarizing force and the large mass causes the heavy ion to have large linear and angular momenta. Thus, in a nuclein- collision, a heavy ion can transfer large amounts of energy and angular momentum to the target nucleus, allowing the study of the nucleus in high excitation and high spin states. The large baryon number also enables the transfer of many nucleons to the target, creating exotic new nuclear species. A heavy ion may also possess a composite character which enhances the transfer of clusters of nucleons, making it useful as a tool of ruclear spectroscopy, in addition to its uses in scattering, fission, and fusion studies (Fisher, 1975).

In addition to the study of nuclei and nuclear reactions by nuclear physicists and chemists, researchers in atomic physics and astrophysics have begun to use heavy ions. Even more recently, heavy ions have received wide-spread applications in biology, medicine, and materials science.

In atomic physics, heavy ions are being used as tools in the $x$-ray emission analysis of elements (Mowat et $a$ Z., 1973) as well as in atomic structure studies of highly stripped ions and their interactions with atoms (Salzborn, 1976). Heavy ions are used in astrophysics to interpret the spectra of stars anci the origins of cosmic rays. Materials science currently makes use of heavy ions in reactor damage simulation studies, ion implantation, and basic solid state research, such as channeling studies (Saltmr.rsh, 1975). Biological and medical interest in heavy ions 
centers on basic radiobiology and radiation therapy (White, 1971).

C. Conventional Ion Sources

In the early heavy ion ecceleration work at Berkeley, a spark-type source was used (York et al., 1946), but most subsequent heavy ion accelerators have utilized an arc-type source. These sources are usually of two types, the Penning discharge source (Penning, 1937) and the duoplasmatron ion source (Von Ardenne, 1956). Both have been developed and modified for heavy ior. production at many accelerator laboratories (Bennett, 1971). Even today they remain essentially unchanged from their initial forms. Both of these ion sources utilize an arc-type electrical discinarge which is collimated by a magnetic field parallel to the axis of the source. This arc discharge results in very short operational lifetimes, and charge state production is limited to less than +10 for practical currents ' of the very heavy elements.

For the acceleration of heavy ions in a linear electrostatic accelerator, the maximum attainable energy per nucleon is directly proportional to the charge-to-mass ratio of the ions, $2 / A$. However, for a cyclotron the maximum energy per nucleon is given by

$$
\frac{E_{\max }}{A}=k\left(\frac{Q}{A}\right)^{2} \text { Mev/nucleon, }
$$

where $k$ is a geometrical constant determined by the size and maximum field of the magnet $(k=147$ at Texas $A \& M)$, and $Q / A$ is the ratio of the charge state to the atomic mass of the ions. Thus in either case, the design and use of heavy ion accelerators is very strongly dependent on the performance of the ion source. In designing such an acceleratox, the size and hence the cost of the device needed to accelerate heavy ions to a given energy decreases as the charge state of the ion being accelerated increases. For a machine alread"r in use, the maximum attainable energy is thus increased as the charge state of the ions is increased.

D. New Ion Source Concepts

The great surge of interest in heavy ions which occurred during the

${ }^{1}$ Practical curreits are defined as $>10^{11} / \mathrm{sec}$ for nuclear studies and $>10^{9} / \mathrm{sec}$ for atomic studies. 
$1960^{\prime} \mathrm{s}$, and the subsequent development of large heavy ion accelerators made obvious the limited technology of the ion sources used for hervy ion production (Iind, 1970), as well as the fundamental impcrtance of the ion source in the design of a heavy ion accelerator (Livingston, 1970). The need for ion source research is still evident, as pointed out by the Report of the Ad Hoc Panel on Heavy Ion Facilities (1974): "We therefore here simply emphasize that the performance of the ion source is critical for the performance of the accelerator complex and, for this reason, the Panel strongly recommends encouragement of ion source research and development." The surge of interest in heavy ions which occurred in the 1960 's had brought about a great amount of development work on conventional ion sources. In addition to this work, research was also begur. on several new ion source concepts including the laser source, plasma source, and electron cloud source. ${ }^{2}$

The laser ion source uses laser irradiation of solid heavy elements to produce highly-charged ions in a very dense expanding plasma. The ion pulses produced by these short laser bursts have very small emittance and are very short, but magnetic mirror confinement can be used to make longer ion pulses (Peacock and Pease, 1969). Although the laser source can theoretically produce large yields of very highly-charged heavy ions (Tonon, 1972), it suffers from a poor duty cycle and problems in the lifetine of the laser, as well as problems involving the extraction of the inighly-charged ions from the laser-initiated plasma burst. The former problem may improve rapidly because of the intense development of fast repetition, high-power lasers for use in fusion studies.

The plasma source, currently developed in several different forms Peacock, Speer, and Hobby, 1969; Zucker, 1970; Alexeff and Jones, 1972; Apard et al., 1973 ), uses energy transfer to an electron plasma by either electron-cyclotron-resonant-heating or by a beam-plasma interaction in a magnetically contained plasma. These sources are all very large and require very large power input to generate and contain the plasmas. In general they have large enittance values that are not very useful for

${ }^{2}$ The development of ion sources of negatively charged heavy ions for use in tandem electrostatic accelerators is not relevant to this work. An excellent review of this work is given by Dawton (1972) and updated by Middleton (1976). 
accelerator injection, and are limited to charge states for heavier elements that are not an improvement over conventional ion sources.

In an electron cloud ion source, a closed or open cioud of energetic electrons is used to create positive ions which are then trapped in the potential well of the electrons while they are stripped of their bound electrons by successive electron impact ionization collisions with the energetic electrons. The electron cloud itself is contained by an externally appiied magnetic field. The HIPAC source (Janes et al., 1966) represents one of the earlier studies of such an ion source. However, the toroidal configuration of the HIPAC was originally intended for fusion research and it suffered from plasma instabilities and difficult ion extraction. The electron ring ion trap (Keefe et al., 1974) also suffers the difficult extraction problem, and must use an electron injector accelerator. In contrast to these closed electron cloud ion sources, the open electron cloud ion source does not suffer this extraction difficulty. The electron beam ion source, which uses a linear electron beam, is such an open electron cloud ion source.

E. Electron Beam Ion Source

The electron beam ion source, as originally proposed and studied by Donets, Ilyushchenko, and Alpert (1968), consists of a very dense energetic electron beam magnetically focussed within a series of cylindrical electrodes placed along the axis of a solenoid. The space charge of the dense cylindrical electron beam cxeates a radial potential well. Positive ions created from a gas or vapor injected into the electron beam are then confined within the beam by this potential well and an axial potential applied to the electrodes at each end of the beam. Successive electron impact ionizations of the confined ions then causes them to become very highly ionized. The ion containment time required for the production of a particular charge state is determined by the density of the eiectron beam. After this period of time, the axial potential trap is removed and the highly-charged ions are axially extracted from the electron beam.

In recent years the EBIS has shown promise as a new approach to the creation of useful beams of multi-charged heavy ions. Theory, as well as preliminary results, show that it is capable of producing high charge 
states of all elements up to uranium. It appears to have a long operational lifetime and a very small emittance, and produces a very stable ion beam. The theory predicts that good yields of very highly-charged heavy ions can be attained by using very dense electron beams within a ve y good vacuum. Also, the EBIS has several variations in its operation which make it adaptable to a particular type of accelerator, as well as useful for other types of studies with multi-charged heavy ions.

The investigation of the EBIS began in 1967 by Donets and co-workers at the Joint Institute for Nuclear Research in Dubna after the work of Redhead (1967) had reported the production of highly-charged ions by electron bombardment of electrostatically confined ions. Experiments performed with a $2.3 \mathrm{keV}$ electron beam of $12 \mathrm{~A} / \mathrm{cm}^{2}$ current density in a $16.5 \mathrm{~cm}$ long solenoid anä at a pressure of $2 \times 10^{8}$ Torr revealed that ions of $\mathrm{C}^{+5}, \mathrm{~N}^{+6}, \mathrm{O}^{+7}$, and $\mathrm{Au}^{+19}$ were extracted from the ion source with a maximum containment time of $10 \mathrm{msec}$. The relative intensities of the charge states of nitrogen measured in their preliminary work indicated that the ionization occurred primarily in successive steps as predicted by theory (Donets. Ilyushenenko, and Alpert, 1969). However, the ionization cross sections deduced from these measurements seemed to be more than five times greater than the theoretical values.

Following the reports of this work, a source of this type was begun in 1970 by the ion source group at the Institut de Physique Nucleaire in Orsay, France. The source at orsay (SILFEC) has produced ions of $\mathrm{C}^{+5}$, $\mathrm{O}^{+6}, \mathrm{~A}^{+14}, \mathrm{Kr}^{+17}$, and $\mathrm{Xe}^{+21}$ with a $2 \mathrm{keV}$ electron beam of $10 \mathrm{~A} / \mathrm{cm}^{2} \mathrm{cur}-$ rent density in a pressure of $10^{-9}$ Torr for a maximum containment time of $180 \mathrm{msec}$ (Arianer et al., 1975). Work is presently continuing on improvement of the SILFEC, with the goal of producing an ion source for the GANIL heavy ion accelerator project in Fronce (Arianer and Goldstein, 1976) with ion currents greater than the present $10^{8}$ to $10^{9}$ ions/sec.

After his earlier success at Dubna with the EBIS, Donets began work in 1971 on a cryogenic version (KRION) of this ion source. This new version was similar to the original EBIS except that a longer, cryogenic solenoid was used. This allowed a larger magnetic field, lower pressures, and low energy consumption of the solenoid in the restricted environment of a high voltage terminal. For containment times of up to $39 \mathrm{msec}$, this 
source has produced ions of $\mathrm{C}^{+-}, \mathrm{N}^{+7}, \mathrm{~A}^{+14}$, and $\mathrm{Xe}^{+29}$ with a $2.5 \mathrm{keV}$ electron beam of $30 \mathrm{~A} / \mathrm{cm}^{2}$ current density in af vacuum of $2 \times 10^{-11}$ Torr (Donets and Pikin, 1974). The containment time of $39 \mathrm{msec}$ was limited by the fact that the electron gun could only be pulsed for a maximum of 40 msec every 2 seconds. This was necessary to allow the cryogenic system time to dissipate the heat generated wi.thin the solenoid by the internal electron gun without letting the superconducting solenoid become normal. The successive ionization cross sections deduced from these measurements for carbon and nitrogen ions have been compared to various theoretical values, and again appear to exceed them (Donets and Ilyushchenko, 1974). The goal in the construction of the KRION was to produce more than $10^{8}$ ions/pulse of bare carbon, nitrogen, and neon nuclei. for injection into the synchrophasatron in Dubna, and it is present? being installed on that accelerator. A new KRION is also being built to study the ionization processes within it (Donets, 1976).

Work began also during 1971 by the ion source group at the Institut für Kernphysik in Giessen, Germany, on a different approach to the EBI: which would allow a continuous extraction of the ions. This group used a strong fiela ionizer of the type developed by Glavish (1968) for a polarized ion source to show 'shat multi-charged xenon ions could be produced by injecting an atomic :er on beam instead of tive hydrogen beam normally used in polarized ion sources. Using this experience, they built a modified strong field ionizer to be used as an EBIS (clausnitzer, Klinger, and Salzborn, 1973). This ionizer uses the electron beam to radially trap the ions and to further ionize them as in any other EBIS. but differs in that the atoms are ionized by electron bombardment only during their axial drift through the ion source. The atoms are injected through the electron gun as an atomic beam and arift through the ionization region to the jon extractor. The axial potential on the electrodes reflects the electrons rather than the ions so that a large electron density can be attained within the ionization region. With a $4.2 \mathrm{keV}$ electron beam of $10 \mathrm{~A} / \mathrm{cm}^{2}$ current density and a transit time of $1 \mathrm{msec}$ in the vacuum of $5 \times 10^{-8}$ Torr, icns of up to $\mathrm{Kr}^{+10}$ and $\mathrm{Xe}^{+13}$ have been observed (Clausnitzer et aZ,., 1975). This ion source is now being used extensively for measurements of atomic charge exchange cross sections of 
highly-charged ions passing through various gases (Klinger, Muller, and Salzborn, 1975) and has delivered nanoampere noble gas ion beams for over several hundred hours. Measurements on this ion source and comparisons with model calculations have indicated that successive ionization is the dominant process taking place in the production of the highlycharged ions (Clausnitzer et al., 1976).

A similar approach to a continuous operation EBIS began at the Institut für Angewandte Physik in Frankfurt, Germany in 1973. This time-of-flight EBIS (TOFEBIS) also does not confine the ions along the axis but does use the electron beam to ionize and radially contain the ions. However, in the TOFEBIS the ions are injected axially as an atomic beam at the electron collection point, and are extracted through a small hole in the cathode of the electron gun, just the reverse of the ion beam flow at Giessen. The work on this EBIS concept is currently in progress, with the design goal being to build an ion source for highlycharged heavy ions to inject in the UNIIAC linear accelerator in Darmstadt, Germany (Becker and Klein, 1976; Kleinod et al., 1976).

Still another approach to the EBIS has recently been undertaken at the Bhabha Atomic Research Centre in Bombay, India by Jain and Divatia (1975). By applying an rf-resonant voltage to the electron beam, they hope to run the EBIS at much higher pressures than normal by throwing out the unwanted low charge states formed in the electron beam from the residual gas. This work has just begun, and the construction of this device, the resonant EBIS (REBIS), and the testing of this concept is yet to come.

At the Cyclotron Institute of Texas A\&M University, feasibility studies on using an EBIS as an external heavy ion source for the 88-inch variable energy cyclotron began in the fall of 1972. The construction of an axial injection system for the polarized ion source had given the impetus for increasing the heavy ion energies from the cyclotron by the use of this new type of external ion source. The design and construction of the Texas A\&M EBIS began in January, 1974. Construction of a full scale test model, as well as a shorter test model were undertaken. These models were designed to be as flexible as possible in form in order to test the operation of the EBIS in several modes, and to study the 
mechanisms of containment and ionization within this type of source. The production of an ultrahigh vacuum within the EBIS and the formation and focussing of a very dense electron beam are stressed in the design of this device in order to achieve ion outputs useful fur cyclotron injection. Cryopunping of the ionization region in the EBIS and an electrostatically convergent electron gun placed external to the solenoid field were the methods proposed and carried out in order to achieve these conditions. Since construction of this ion source began in 1974 , these methods have also been adopted in the EBIS research at other laboratories (Donets and Ilyushchenko, 1974; Becker, Klein, and Kleinod, 1974; Arianer, MacFarlane, and Ulrich, 1975). 


\section{A. Operating Principles}

The EBIS is a linear ion containment device. The jasic cunfiguration of such a source is shown schematically in Fig. I(a). A very dense electron bearn is magnetically focussed through a series of cylindrical electrodes placed along the axis of a solenoid. The potential distribution, shown in Fig. $l(b)$, is applied to this series of insulated electrodes to prevent the axial escape of the positive ions created by the electron beam. The negative space charge of the electron beam produces a potential well which radially traps the positive ions. The depth of this well can be calculated from Gauss' law assuming a uniform cylindrical electror, beam of radius $x^{\prime}$ ' and is given by

$$
\Delta V=\frac{480 I_{e}}{E_{e}^{1 / 2}}\left\{1+2 \ln \frac{R_{T}}{r_{f}}\right\} \text { volts }
$$

where $I_{e}$ is the electron beam current in $A, E_{e}$ is the energy of the electrons in $\mathrm{kev}, r_{T}$ is the radius of the drift tubes, and $r_{f}$ is the radius of the electron beam. The minimum in this potential well occurs on the axis of the electron beam, and its variation is essentially parabolic within the beam, and logarithnic between the beam and the drift tube (Smith and Hartman, 1940).

The positive ions created by electron impact ionization collisions of the electrons with neutral atoms inside the drift tube are thus trapped in this potential well. These ions can then be confined within the electron beam by this potential well until they have become highly ionized by further electron impact ionization collisions. After this confinement period the ions are axially extracted from the electron beam by lowering the axial potential applied to one end of the ion source, as shown in Fig. $1(\mathrm{~b})$. The electrons and ions are separated in the fringing field of the solenoid by the negative potential of tile ion extractor. As a result of this axial extraction of the confined ions from within the small 

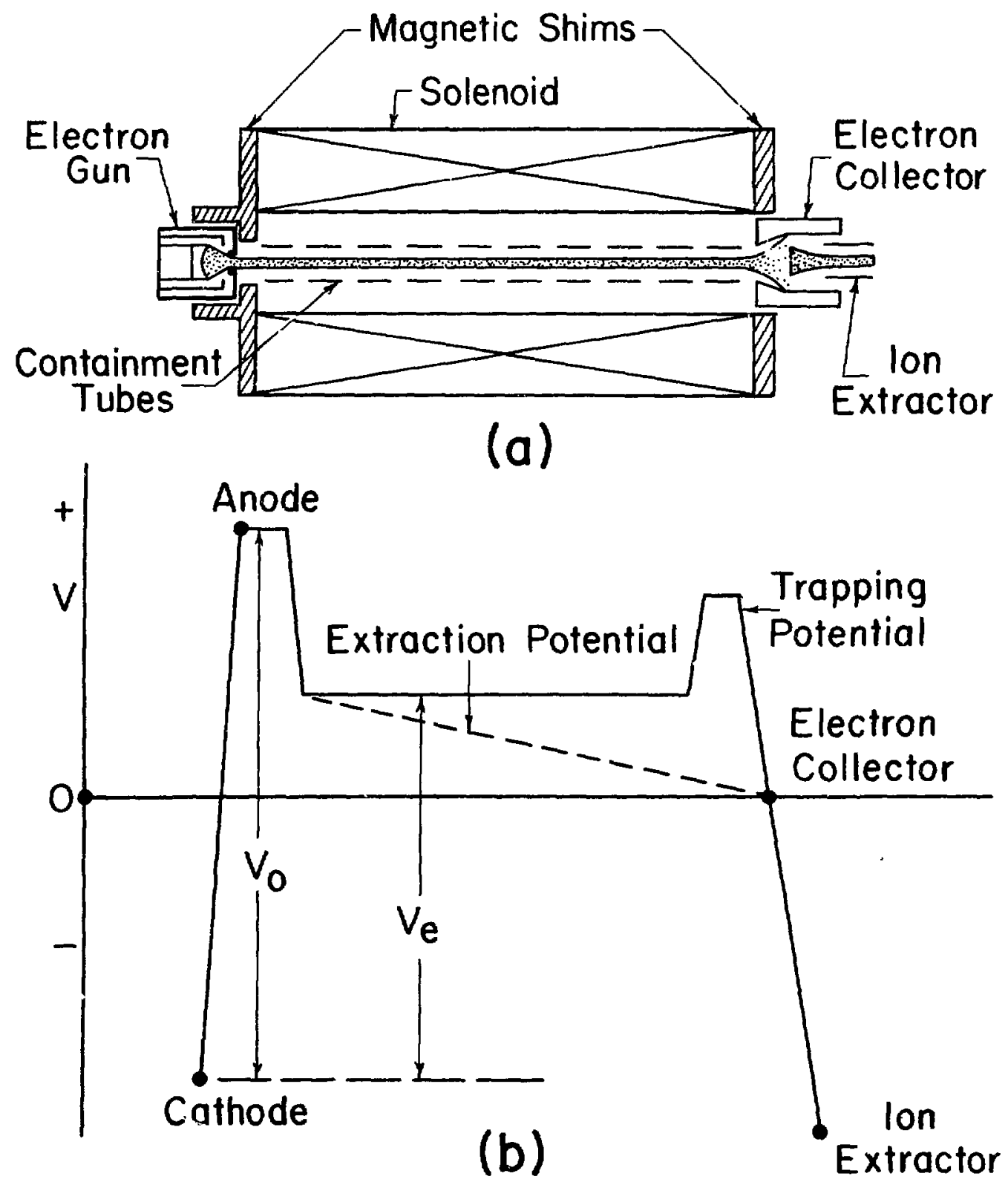

FIG. 1. (a)Basic configuration of the EBIS; (b)Applied axial potentials during containment and extraction. 
diameter electron beam, an ion beam of a very small emittance is produced. B. Ionization T".eory

Several assumptions are made in order to predict the EBIS output. First, one assumes a monoenergetic, uniform density electron beam with complete axial symmetry of the beam and the magnetic and electric fields. Then, as has been shown experimentally (Donets, Ilyushcheiko, and Alpert, 1969; Arianer et a2., 1975), the main ionization process is successive electron impact ionization collisions. Electron-ion recombination is calculable and known to be negligible, and ion-ion charge transfer losses are neglected because of their large coulomb repulsion. Finally, the ions are assumed to be distributed uniformly throughout the ionization volume of the electron beam with a Maxwellian energy distribution.

The first assumption simplifies calculations for the electron beam in the EBIS and, though it is not completely correct, it enables one to give a rather good description of the ion source output. The electron beam has only a very small energy spread due to thermal motion anc this causes a variation of the beam density with radius. However, only the potential well shape is changed, not the well depth, and axial symmetry still exists.

The validity of the important second assumption, the step-by-step ionization of the trapped ions, can be seen from the experimental measurements of electron ionization cross sections (Schram, 1966). These data show that the multiple ionization of an atom is approximately an order of magnitude less likely than a single ionization for each additional electron being removed during the collision. Thus, if one knows the cross section, $\sigma_{i}\left(E_{e}\right)$, for going from charge state $(i-1)$ to charge state $i$ at the electron energy $\mathbb{T}_{e}$, one can calculate the average time required for this transition to have unit probability by

$$
\tau_{i}=\frac{e}{j_{e} \cdot \sigma_{i}\left(E_{e}\right)}
$$

where $j_{e}$ is the electron beam current density in $\mathrm{A} / \mathrm{cm}^{2}$ and $\mathrm{e}$ is the charge on the electron. The mean containment time necessary to produce a specific charge state $q$ is then given in this simplifjed model by suming over 
the transition times for all the charge states up to $q$ :

$$
\mathrm{\tau}_{q}=\frac{\mathrm{e}}{\vec{j}_{e i=1}} \sum_{i=1}^{q} \frac{1}{\sigma_{i}\left(E_{e}\right)} \text {. }
$$

Calculation of these containment times for the production of certain charge states of a specific element requires knowledge of the ionization cross sections, $\sigma_{i}\left(E_{e}\right)$. These values are known experimentally for only a few multi-charged ions, so they must be calculated in most cases. Theoretically, no exact description of this quantity exists for all electron energies, charge states, or elements. The general formulation that has been used for these cross sections is the Bethe (1930) approximation:

$$
\sigma_{i} \simeq 1.63 \times 10^{-20} \sum_{i} \sum_{e} \frac{\ln \left(E_{e} / I_{i}\right)}{E_{e} I_{i}},
$$

where $I_{i}$ is the ionization energy of the $i$ th electron of the ion in kev. This formula was used for the HIPAC calculations (Janes et al., 1966), but is only valid for electron energies that are much greater than the ionization energies of the electrons being removed $\left(E e^{\gg}\right) I_{i}$, for $i=1,2, \ldots$, q). Becker, klein, and Schmidt (1972) have modified this description to make it agree with the known experimental cross sections near the maximum by dropping the summation, nultiplying by a factor of ten, and using the ionization potential for the most easily removed electron when going from the $(i-1)$ th to the $i$ th ionization state:

$$
\sigma_{i} \simeq \frac{1.63 \times 10^{-19}}{E_{e} \cdot I_{i}} \ln \left(E_{\epsilon_{i}} / T_{i}\right)
$$

In addition, the functional dependence of the electron impact ionization cross sections has been represented by several empirical formulas. The work of Drawin (1961) is one such representation that has been used, but does not reproduce the known cross sections within experimental errors. Then Lötz (1967a, 1967b, 1968) used the work of Rudge and Schwartz (1965) and the data collected by Kieffer and Dunn (1966) to derive the semi-empirical formula: 


$$
\left.\sigma_{i}=\sum_{i=1}^{\mathrm{N}} \frac{a_{i} \cdot q_{i}}{E_{e} \cdot I_{i}} \ln \left(E_{e} / I_{i}\right)\left\{1-b_{i} \exp _{i}\left(1-E_{e} / I_{i}\right)\right]\right\}
$$

where the summation is over the $\mathrm{N}$ subshells of the atom or ion, $a_{i}, b_{i}$, and $c_{i}$ are constants taken from experimental data, and $q_{i}$ is the number of equivalent electrons in the $i$ ith subshell. The fits of the Lötz formula to some of the data reported by Kieffer and Dunn (1966) are shown in Fig. 2. Most experimental data can be approximated by this formula within 10: over the whole energy range between the timeshold energy and $10 \mathrm{kev}$, and all experimental results can be approximated rithin experimental errors. This success was the basis for the use of the Lötz semi-empirical formula in the theoretical projections made in the present work (Hamm and Kenefick, 1973). The extrapolation of this formula to high charge states has been shown to be valid in this energy range for several different elements (Donets and Ilyushchenko, 1974; Donets and Pikin, 1975). In order to calculate the ionization cross sections with this formula, the successive ionization potentials of the electrons of the elements are needed. Once again, only a small number of these potentials have been experimentally measured (Mocre, 1970). These numbers have been calculated by carlson et al. (1970) for all charge states of all known elements with an approximation formula using only the quantum data frum the neutral atoms of twe elements. The values calculated in this manner are in good agreement with the available experimental data, and have been shown to be in good agreement with Hartree-Fock-slater calculations for very highly-charged heavy ions (Becker, Klein, and Schmidt, 1972). Using these values for the successive ionization potentials and the Lötz relationship (Eq.(2.6)) for the successive ionization cross sections, one can theis use Eq.(2.3) to calculate the mean ionization time required for a particular charge state $q$. Fig. 3 shows such a calculation for several charge states in krypton, showing the normalized containment time, $\tau_{i}^{\prime}\left(j_{e} \equiv 1\right)$, as a function of the normalized electron energy, $\vec{E}_{e} / I_{i}$. The minimum in each curve occurs near the electron energy that corresponds to the maximum in the cross section for the removal of the last electron. This maximum in the ionization cross sections is seen experimentally to 

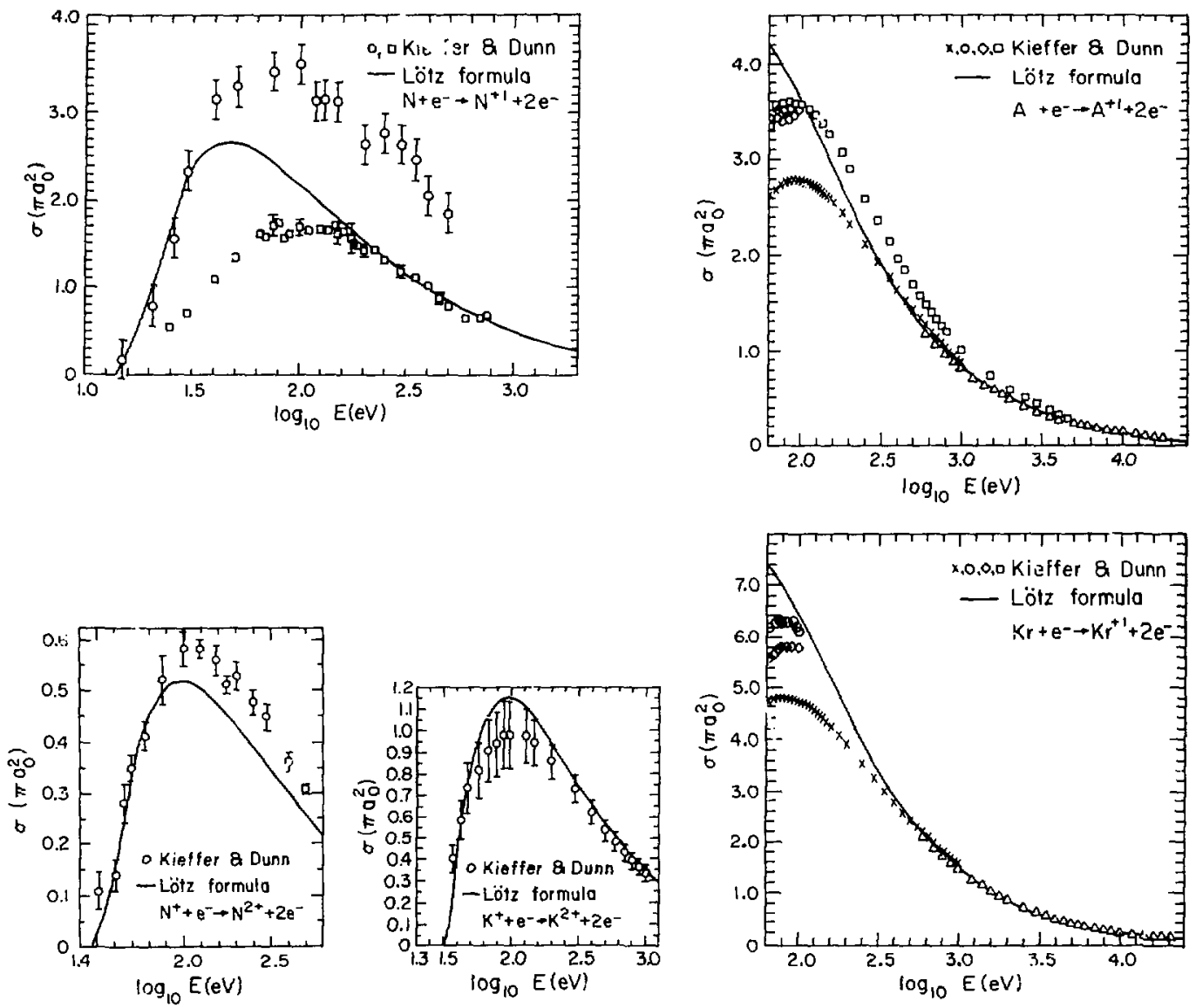

FIG. 2. Comparison of the Lötz prediction of the ionization cross sections with measured cross sections from kieffer and Dunn (1966). 


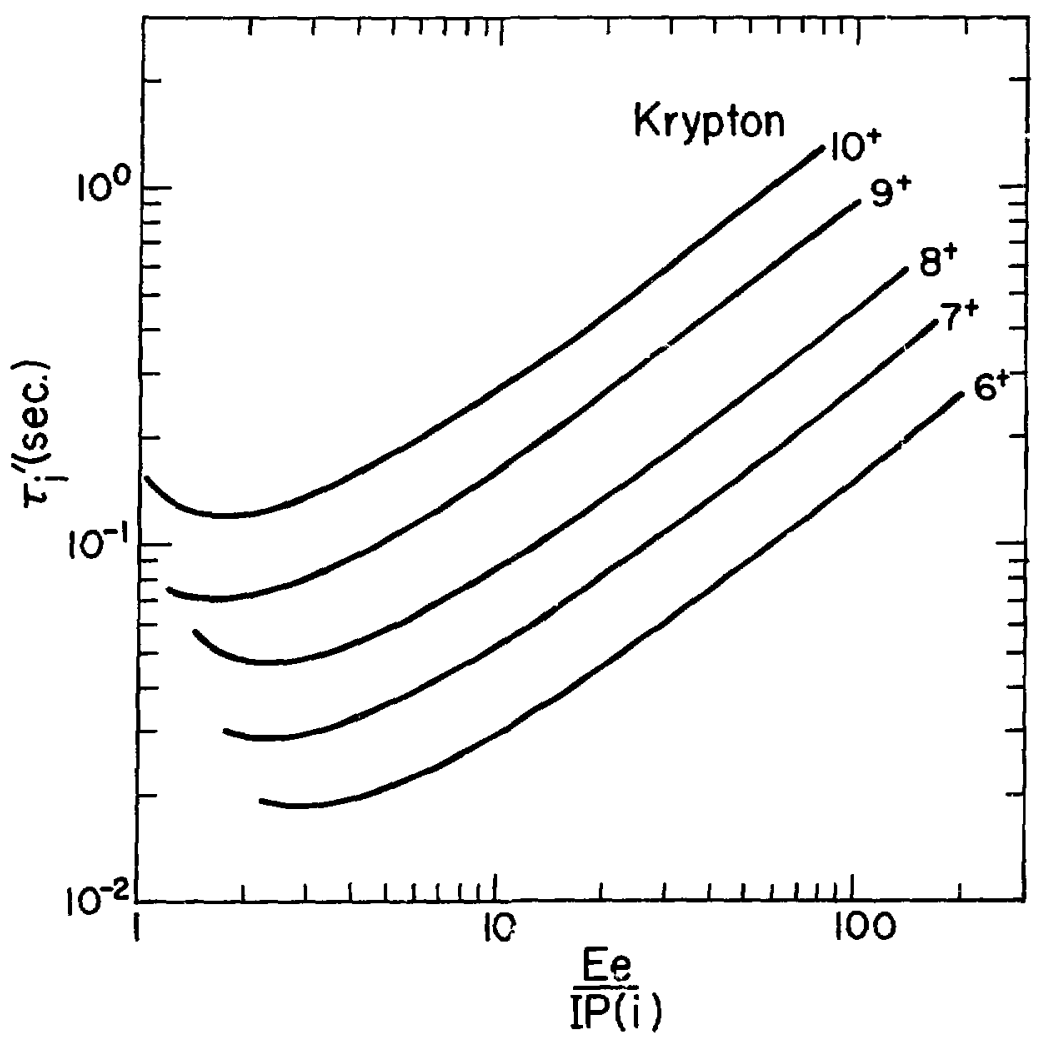

FIG. 3. Normalized containment time for several charge states of krypton as a function of the nomalized elactron energy. 
occur at 22 to 3 times the ionization energy of the particular electron being removed. This fact can then be used to minimize the containment time for a particular charge state by an appropriate choice of the electron beam energy.

Since others have shown the validity of this simplified model, one can also study the time evolution of all of the charge state abundances of ar. elenent by solving the set of differential equations given by:

$$
\frac{d C_{i}}{d t}=k_{i} C_{i-1}-k_{i+1} C_{i}, \quad i=0,1, \ldots, q
$$

where $C_{i} \equiv N_{i} / N_{0}$ and $k_{i} \equiv j_{e} \sigma_{i} / \mathrm{e}$, with the condition that $C_{0}(t=0)=1$ and $C_{i}(t=0)=0$ for $i \geqslant 1$. Here $N_{i}$ is the number of ions of charge state $i$ and $A_{0}$ is the number $\mathrm{c}$ in initial neutral atoms present. The value of $q$ is given by either the number of electrons in the atom (z) or by the maximum degree of ionization obtainable with the electron energy being used, this being the last electron when $E_{e}>I_{i}$

The results obtained from these equations for the ions of argon, assuming an electron beam energy of $2.5 \mathrm{keV}$, are presented in Fig. 4. The charge state distribution is plotted as a function of the normalized containment time $\tau_{i}^{\prime}$, which is the containment time in seconds for a current density of $1 \mathrm{~A} / \mathrm{cm}^{2}$. The normalized containment time found from $\mathrm{Eq}$. (2.3) for $A^{+8}$ at the electron energy given here is indicated in the figure by the arrow. The proportionality of the ionization cross section to the number of equivalent electrons. in the electron shells is clearly seen in argon by the break in the charge state distribution for the +5 and +14 ions. The distribution of the +15 and +16 ions is due however to the fact that the last electron that can be removed in argon by a $2.5 \mathrm{keV}$ electron is the 16 th one. The two remaining electrons in the innermost shell are bound too tightly to be removed by this energy.

Additional parameters can be added to this simple ion time evolution theory to account. for the continuous injection of atoms and other ionization processes. Such calculatjons have been done by Baron (1973), Darling, Mncinan, ani Davis (1972), and Salop (1973, 1974). The effect of these auditional parameters on the EBIS measurements in this work will be described later. 


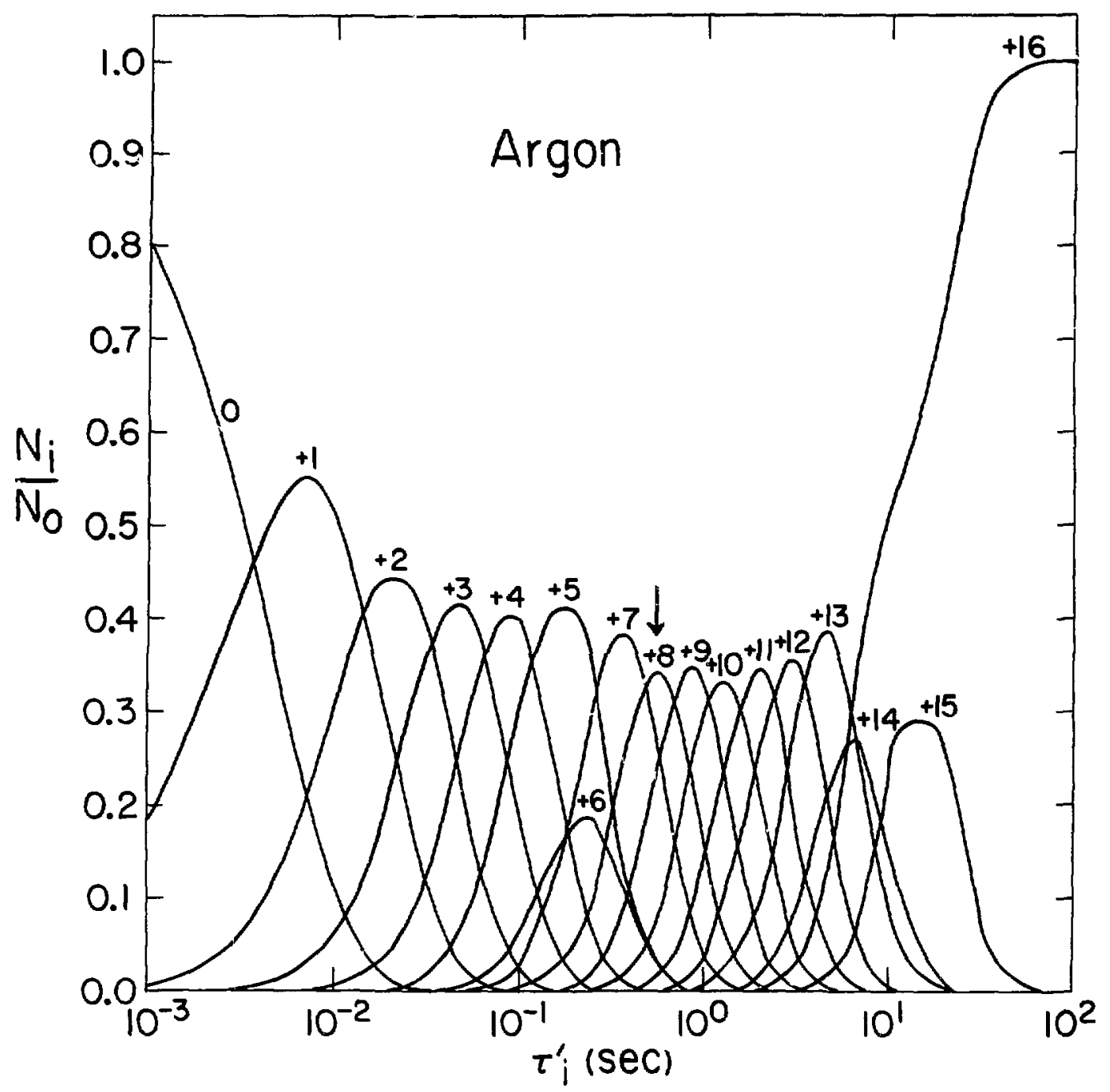

FIG. 4. Time evolution of argon ions for successive ionization with a $2.5 \mathrm{keV}$ electron beam. 
Using the above assumptions for this successive ionization theory, one ignores the other production and loss mechanisms and calculates the upper limit of the ion production within this ion source by assuming that the ion charge density is equal to the electron beam charge density throughout the ionization volume at the end of the containment time $\tau_{q}$ for maximum production of the $q$ th charge state. The average current for the charge state $q$ is then found by taking the product of the electron charge density and the ionization volume, multiplying by the fraction of total ions found in charge state $q$, and dividing by the time required for each ion pulse. This yields as the expression for the maximum current of particles with charge state $q$ :

$$
I_{q}=5.28 \times 10^{-4} \frac{f}{\beta} \frac{j_{e}^{I} e^{2}}{\tau_{q}^{\prime} E^{1 / 2}} \quad \mu \mathrm{A},
$$

where $Z$ is the ionization length in $\mathrm{cm}$, and $\tau_{q}^{\prime}$ is the normalized containment time in seconds for the maximum production of charge state $q$. The quantity $f$ is the ratic of the charge density of ions with charge $q$ to the total ion charge density, and $\beta$ is the correction factor which accounts for the entire ion production cycle, including the neutral atom injection time $\tau_{\text {inj' }}$, the containment time $\tau_{q}$ ' and the extraction time $\tau_{\text {ext }}$. This correction factor is then given by

$$
\beta=\frac{\tau_{\text {inj }}+\tau_{q}+\tau_{\text {ext }}}{\tau_{q}}
$$

and is always a quantity greater than unity.

The value for the quantity $f$ can be determined for any charge state at its maximum value from the solution of Eq. (2.7) as shown in Fig. 4 (p. 19). Thus, for a given ionization length and set of electron beam parameters, one can then solve Eq. (2.8) by calculating $\tau_{q}^{\prime}$ with the aid of Eq. (2.3). The results obtained from such a calculation are shown in Fig. 5 for parameters obtainable with the Texas A\&M prototype EBIS. The charge states in the calculation were chosen such that the final energy of these ions accelerated on the Texas A\&M cyclotron would be $10 \mathrm{MeV} /$ nucleon. The electron gun extraction voltage was set at $7 \mathrm{kV}$ and the atom injection 


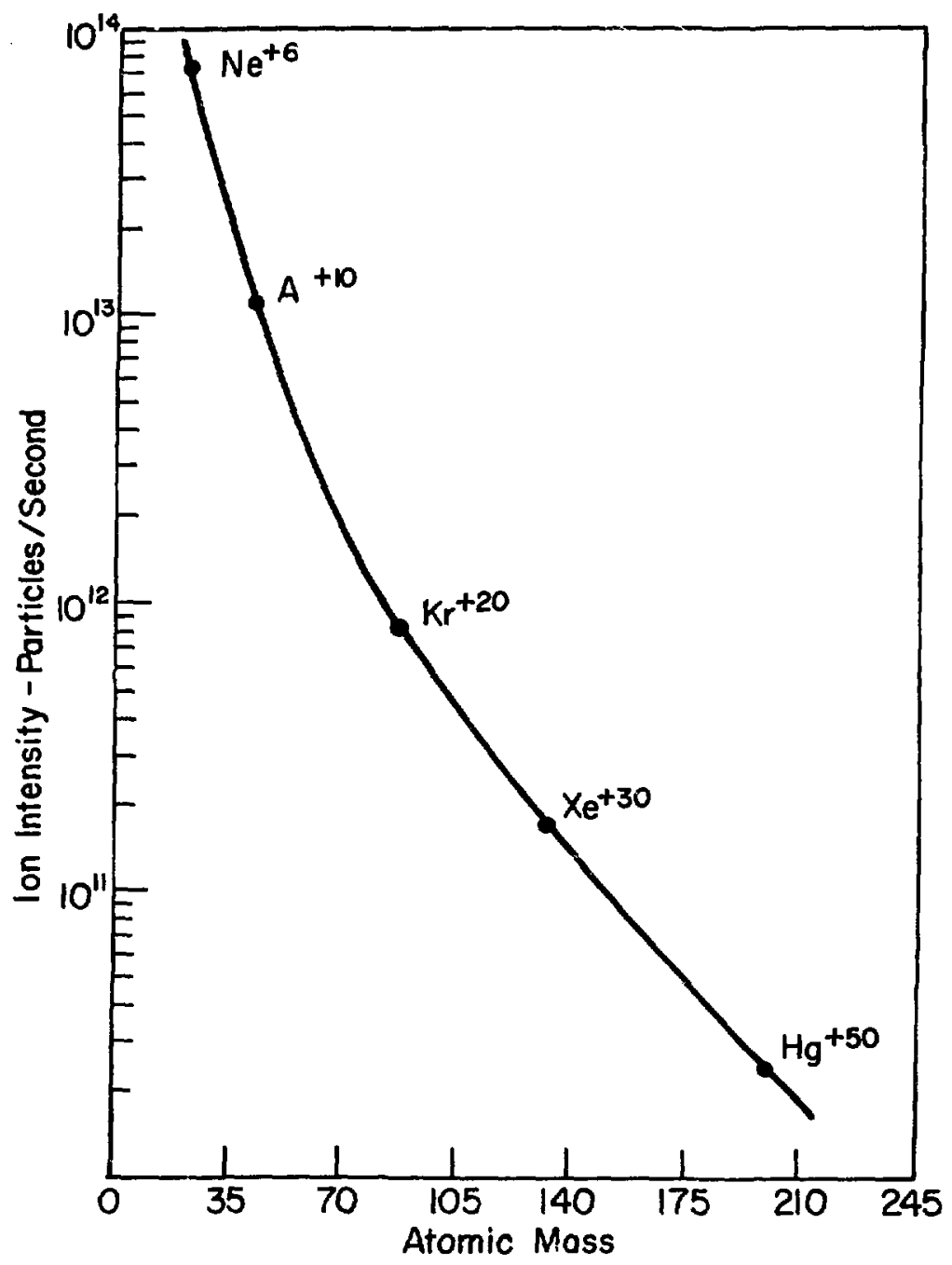

FIG. 5. Estimated upper limit currents for the Texas M\&M prototype EBIS. 
and ion extraction times were chosen to be 100 llsec. Extraction of the ions from the one meter long electron beam was assumed to be complete, and residual gas contributions to the ion output were assumed to be negligible. One now turns to the assumption of a negligible background gas contribution, which can readily be translated into a vaclium requirement within the EBIS.

\section{Vaclum Considerations}

The background gas atoms are also ionized and trapped by the electron beam and will thus cause the electron space charge to be neutralized more rapidly. If one assumes that the ions created from the residual gas are a fraction $C$ of the total ions trapped in the beam at the neutralization time, then the residual pressure $P_{r}$ within the ionization region can be shown to be given by

$$
\mathrm{P}_{r} \leqslant 1.5 \times 10^{-10} \frac{C \cdot j_{e}}{\sigma_{r}\left(E_{e}\right) \tau_{q}^{\prime} \cdot E_{e}{ }^{1 / 2}} \text { Torr, }
$$

where $\sigma_{r}\left(E_{e}\right)$ is the cross section for the ionization of the background gas in units of $10^{-16} \mathrm{~cm}^{2}$. In a baked ultrahigh vacuun system, the main residual gas is the hydrogen evolved from the walls of the system (Wheeler, 1972). Thus, the ionization cross section for hydrogen can be used for $\sigma_{r}\left(E_{e}\right)$

Another vacuum requirement is given by the charge exchange between highly-charged ions and the residual gas atoms during their ionization, extraction, and transport. The charge exchange within the ionization region has been shown to yield a pressure limit of

$$
{ }_{\mathrm{C}}^{\mathrm{P}_{\mathrm{C}}} \leqslant 3.0 \times 10^{-7} \frac{\dot{j}_{e}}{\mathrm{\tau}_{q}^{\prime}} \text { Torr }
$$

when the cross sections for the low energy ions are assumed to be given by atom-atom collisions (Becker, Klein, and Schmidt, 1972). As for the charge exchange during extraction and transport of the ions, very little is known about the charge exchange cross sections for highly-charged heavy ions at the extraction energies used in the EBIS. Recent experimental data (Crandall, 1977) and extrapolation of a semi-empirical theory 
(Schmelzer, 1970) indicate that these cross sections will be at worst $10^{-14} \mathrm{~cm}^{2}$. Using this estimate; the vacuum requirement for extraction and transport of the ions can be calculated from

$$
P_{t} \leqslant-3.03 \times 10^{-5} \frac{\operatorname{In} T}{L} \text { Torr, }
$$

where $T$ is the transmission ratio of ions over the path $I$ measured in $\mathrm{m}$. For an extraction, acceleration, and transport path of $10 \mathrm{~m}$, a transmission of 998 yields a required pressure of $3.0 \times 10^{-8}$ Torr in this region. The requirements for the vacuum in the ionization region cón be demonstrated by a typical case, such as the production of $A^{+12}$ ions at $2.5 \mathrm{kev}$ with a required $j_{e} \cdot \tau_{i}$ of 3.0 . At this energy, $\sigma_{r}=0.09 \times 10^{-16} \mathrm{~cm}^{2}$ (Kieffer and Dunn, 1966), thus if one uses $j_{e}=10^{2} \mathrm{~A} / \mathrm{cm}^{2}$ and assumes the residual gas contamination to be $1 \%$, the following values are obtained from Eqs. (2.10) and $(211): P_{r} \leqslant 3.5 \times 10^{-10}$ Torr and $P_{C} \leqslant 1.0 \times 10^{-5}$ Torr.

Clearly, the first requirement calls for ultrahigh vacuum techniques within the ionization volume, with the ultimate residual pressure determining the residual gas contribution to the total ion output of the ion source. The second requirement then serves as an upper limit to the pressure of the working gas that can be used in the ionization volume. The importance of this pressure limit in describing the ion production within the electron beam will be examined later in this chapter. Finally, the transport pressure limit $P_{t}$ shows that ultrahigh vacuums are also needed in the ion transport system to obtain complete transmission of the extracted ions. Though the transport vacuum required is not nearly so severe as the ionization region residual pressure, it must not affect the operation of the ion source with the electron beam on and the working gas being injected into the source. This means that the pumping impedance must be high between these two regions, or differential pumping must be used.

\section{Electron Beam Focussing}

The containment time required for the ions in the EBIS to be able to reach a given charge state is inversely proportional to the electron beam current density, while the number of ions available per pulse from the EBIS is directly proportional to the electron beam current. Maximum output from this ion source thus calls for maximizing both of these 
quantities. However, an additional constraint on this procedure consists of keeping the electron energy near the maximum of the ionization cross section of the charge states desired.

Since most electron guns operate under space-charge limited conditions to minimize current fluctuations due to changes in the temperature of the eritting surface, the current emitted from an electron gun is given by

$$
I_{e}=P \cdot V_{0}^{3 / 2} \quad A,
$$

where $V_{0}$ is the voltage applied between the electron gun anode and cathode in $\mathrm{V}$, and $P$ is a geometrical quantity for the gun in $A / V^{3 / 2}$, defined as the "perveance". For a given electron gun design and gun voltage, the electron bearn current is thus fixed, so the only method of increasing the electron beam current density is to decrease the size of the electron beam. Since there are several types of electron guns of varying perveance and convergence, there are several methods available for injecting high current, high density electron beams along the axis of a focussing solenoid.

The simplest method is from an electron gun placed inside the solenoid along the axis of the uniform magnetic field. This type of electron focussing is known as immersed or confined flow, and gives an electron beam that has the same diameter as the cathode. The beam envelope has an outward scalloping structure, with the magnitude of the scallops inversely proportional to the focussing field of the solenoid. Thus, the maximum current density that can be obtained is equal to the emission current density of the cathode when a very large focussing field is used, and such emission densities never exceed $100 \mathrm{~A} / \mathrm{cm}^{2}$ (Jenkins and Trodden, 1965).

Another type of confined flow is that in which the electron gun is placed on the axis of the solenoid in the fringe field so that the electron trajectories follow the magnetic field lines. Thus the electron beam is "compressed" and the current density achieveable from such a semi-immersed electron gun is greater than the totally immersed gun by the convergence ratio of the fringing field, usually only a factor of 2 or 3. The beam still has a scalloped structure, and requires a very 
large focussing field. The properties of both types of immersed gun electron beams have been very thoroughly studied because of their use in electron beam tubes (Ivey, 1954; Susskind, 1956; Dow, 1958; Beck, 1964).

The third method of magnetic beam focussing uses a Pierce type convergent electron gun (Pierce, 1954; Brewer, 1957) placed external to the bore of the solenoid and shielded from the magnetic field. This type of focussing, founded on principles given by Brillouin (1945), achi. . ss a smooth beam by a balance of the space-charge, centrifugal, and Lorentz forces acting on the electrons. The use of the electrostatically convergent electron gun in this manner yields current densities of up to several hundred $\mathrm{A} / \mathrm{cm}^{2}$, values which are much higher than those for immersed flow. This method is usually called Brillouin flow, or space-charge-balanced flow, and has also been very thoroughly studied bezause of its extensive use in traveling-wave electron tubes (Wang, 1950; Mendel, 1955; Brewer, 1959). The properties of these beams have been studied both theoretically and experimentally (Smith and Hartman, 1940; Cutler and Hines, 1955; Cutler and Saloom, 1955; Herrmann, 1958; Amboss and Gallagher, 1964).

By using the equations of motion for the electrons in a magnetic field, Busch's theorem, and Gauss' theorem, one obtains the axial magnetic field necessary to focus the electron beam in each type of flow:

$$
\mathrm{B}_{0}=1.477 \cdot \mathrm{C} \frac{\mathrm{I}_{e}^{V_{2}}}{V_{e}^{1 / 4} \cdot r_{f}} \mathrm{kG} \text {. }
$$

where $V_{e}$ is the electron beam voltage in $\mathrm{kV}, r_{f}$ is the focussed electron beam radius in $\mathrm{mm}$, and $\mathrm{C}$ is a function that is determined by the type of flow being used. For Brillouin flow, $\mathrm{C}=1$; for the semi-immersed flow, $C=\left\{1-\left(\psi_{c} / \psi_{0}\right)^{2}\right\}^{-1 / 2}$, where $\psi_{c} / \psi_{0}$ is the ratio of the magnetic flux through the cathode of the electron gun to the:magnetic flux through the focussed electron beam. The completely immersed flow yields $\mathrm{C}$ as a calculable funct: on of $\left(r_{\max }{ }^{-r_{f}}\right) / r_{f}$, where $r_{\max }$ is the maximum radius of the focussed beam. This value can be computed numerically. For instance, using $\left(r_{\max }{ }^{-r} f\right) / r_{f}=0.1$ yields $\mathrm{C}=4$, and $\left(r_{\max }{ }^{-r_{f}}\right) / r_{f}=0.01$ yields $\mathrm{C}=25$. These results show that Brillouin flow is the most efficient form of focussing in terms of the magnetic field strength requized. In addition to this, 
the smooth zylindrical bean and high current density obtainable with this method make it the most desirable form for use in an EBIS.

In the case of Brillouin flow, the electron gun cathode and anode are shielded from the field of the focussing magnet, and the electron beam is ideally injected into the steeply rising magnetic field. The shielding is always imperfect because it must contain an aperture for the beam to pass through, so the magnetic field does not actually have the shape of a step function. The study of this transition region in the focussing system, and the placement of the electron gun and magnetic shield to achieve the conditions of Brillouin flow has been performed by several groups. Molnar and Moster (1951) have studied the relationship between the magnetic field strength, aperture radius, beam radius, and shield position necessary for Brillouin flow. Other theoretical and experimental studies have been conducted by Müller (1953), Bevec, Palmer, and Susskind (1958), and Shimada and Nishimaki (1969). These studies all show that the optimum electron gun and magnetic shield positions are such that the electrostatic minimum of the electron gun in the absence of a focussing field should occur at the point on the axis where the magnetic field has fallen off to about $70 \%$ of its full value. This value is presently accepted by most designers of Brillouin flow systems. This entrance condition is relatively insensitive to the form of the build-up of the magnetic field through the shield. However, the theoretical description of the magnetic field variation in the transition region that most correctly describes the actual fiela on the axis of a hole of radius $a$ in $a$ thin magnetic shield of high permeability with a uniform field $B_{0}$ on one side of it is given by

$$
\frac{B_{z}}{B_{0}}=\left(\begin{array}{l}
1-\pi^{-1}\left\{\operatorname{ta}^{-1}(a / z)-\frac{z / a}{1+(z / a)^{2}}, z / a>0\right. \text { (Inside shield) } \\
\pi^{-1}\left\{\tan ^{-1}(-a / z)+\frac{z / a}{1+(z / a)} 2\right\}, z / a<0 \quad \text { (Outside shield) }
\end{array}\right) \text { (2.15) }
$$

where the origin, $z / a=0$, is located at the inside face of the shield, and the thickness of the shield is unimportant as long as it is thick enough to avoid saturation.

The magnetic field build-up in the transition region can also be 
used in Brillouin flow to further converge the electron beam as it traverses this region. By a suitable choice of electron gun placement and magnetic field build-up, the electron beam is adiabatically compressed by the increasing magnetic field. This method of electrostatic compression followed by magnetic compression was first proposed by Pierce (1954).

The resultant total beam area compression can be very high, and the magnetic field required for focussing is the least possible for a given beam, but the entrance conditions for a well focussed laminar beam are rather sensitive. Experimental, as well as theoretical studies have been conducted on this method. Geppert (1960) first reported work with a total area compression of an electron beam of 130 to 1 , with a 16 to 1 compression by the magnetic field. A total area compression of an electron beam of 1350 to 1 has been reported by Kikushima and Johnson (1963), and experimental studies on magnetic compression have been reported by Vaidya and Ghandi (1964), Amboss (1969), and Seeger (1969). These studies show that electron beam current densities of greater than $1000 \mathrm{~A} / \mathrm{cm}^{2}$ are possible with this method of focussing.

The problems involved in correct placement of the electron gun and magnetic field build-up have now been minimized for designers of electron beam focussing systems by the advent of digital computer analysis programs that very closely follow the experimental results observed in these systems. Thus a designer can numerically study the effect of changes in a focussing system very easily and produce rather quickly a system that yields a well focussed beam. One early program capable of this type of work was written by Boers (1965), while the most widely used electron beam design program is by Hermannsfeldt (1973).

While the Pierce-type electron gun used for the present EBIS work was designed by the manufacturer with the aid of the Hermannsfeldt program, the placement of the magnetic shield and electron gun in the EBIs was studied here theoretically with the Boers progxam, since the laboratory computing facility dictated the use of the less complex computer code. However, calculations repeated on the electron gun design with this code duplicated the results obtained by the manufacturer using the more complex code by Hermannsfeldt. Predictions of beam focussing and theoretical fits to experimentall 7 measured beam properties calculated 
with Boers' code are presented in a later chapter.

E. Ion Containment Dynamics

The influence of positive ions created within a magnetically focussed electron veam was mentioned in work by Pierce (1944), and the trapping and extraction of these ions from within an electron beam has been studied by Hines, Hoffman, and Saloom (1955) with an EBIS-lije structure (the figure on p.1160 of this reference is similar to Fig. 1, p. 12 of the present work). Their work is an extension of earlier work by Spangenberg, Field, and Helm (1947) and they have studied the pressure limitations for complete space-charge neutralization of an electron beam both theoretically and experimentally. The neutralization pressure has also been studied by Hopson (1963) as the required pressure for the electron beam to generate a plasma. Both of these investigations used magnetically focussed cylindrical elect:on beams that produced singly charged ions from the neutral atoms injected into the device. The successful results of these analyses imply trat this EBIS can be analyzed in a similar way in order to determine the dynamics of the ion containment.

According to the classification of electron streams by Lawson (1959) or Harrison (1963), the EBIS is also just a neutralized electron beam since the streaming motion of the electron beam predominates; that is, the probability of a primary collision is $\ll 1$ for electrons in the beam of the EBIS. This is in contrast to a plasma stream where the thermal motion of the electrons is as important as the streaming motion. Since such a neutralized stream is influenced by the collective properties of the free charged particles in the applied electric and magnetic fields, the mathematical tools of plasma physics can be used to study this system. A large amount of material already exists about the behavior of such streams from other devices that use electron beams, and the experiments on these devices are essentially in good agreement with theoretical treatments. Such neutralized beams are on the whole less complicated than neutral plasmas. Among the processes that occur in such beams are atomic collisions and transport phenomena, as well as the collective phenomena of space-charge flow and charge screening. 
The appropriate parameters to characterize the EBIS "stream" are the kinetic temperatures, number densities, fundamental lengths, and natural frequencies of the constituents. If one assumes that the ionization of residual gases is negligible, the charged constituents in the EBIS are the beam electrons, the secondary electrons emitted caring ionization, and the ions being confined in the electron beam. However, it will be assumed initially that the secondary electrons are unimportant in characterizing the EBIS and can be ignored. Later discussions of the processes in the EBIS will show the validity of this assumption.

The concept of temperature is rather difficult to delineate in the EBIS, and is only useful as a calculational parameter. Kinetic theory relates a temperature to the mean translational energy of each constituent in a plasma. Each mode of energy storage car: thus be parameterized by a temperature, so that in a non-equilibrium situation such as the EBIS each constituent can have several temperatures. Also, the difficulty is increased by each constituent having its own temperatures. Usually one denotes the neutral gas temperature as $T{ }^{\prime}$ the ion temperature as $T_{i}$ and the electron temperature as $T_{e}$. In the magnetic field, the charged particles can have temperatures corresponding to motion rarallel or perpendicular to the field, denoted as $T_{h}$ and $T_{\perp}$ respectively. However, since the streaming motion of the electrons predominates in the EBIS, one can neglect the temperature component perpendicular to the magnetic field and use the usual plasma notation (chen, 1973) to give the temperature as:

$$
k T_{e}=2 E_{e} / 3=0.67 \mathrm{~V} e \quad \mathrm{keV},
$$

where $V_{e}$ is the potential through which the electron beam is accelerated in $\mathrm{kV}, \mathrm{T}_{e}$ is in ${ }^{\circ} \mathrm{K}$, and $k$ is Bolczman's constant. The neutral gas atoms to be ionized in the EBIS are assumed to have thermal energies given by $T_{g} \simeq 0.04 \mathrm{eV}$ (Jancel and Kahan, 1966), and the resulting ions are assumed to have temperatures $T_{i}$ that are different for each charge state. An approximate method for determining these temperatures will be examined later, showing that the ion temperatures are only a few ev for all of the ions that are contained in the EBIS.

The number densities of the constituents in the EBIS are denoted by $n_{e}$ for the electrons and $n_{i}$ for the ions. The electron number density 
is given approximately by $n_{e}=j e^{/(e v} e \mathrm{~cm}^{-3}$ if the secondary electrons are ignored, where $v_{e}$ is the electron velocity in $\mathrm{cm} / \mathrm{sec}$. This density is independent of time, whereas the ion number densities, $n_{i}(t)$, are functions of time. The ion densities can be approximately determined by the successive ionization model given in Eq. (2.7). As previously stated, this is only approximate since the ion loss mechanisms have been ignored in tinis calculation. At the end of the containment time ' $q$, one then assumes that quasi-neutrality exists in the homogeneous jeam of the EBIS so tinat

$$
n_{e} \simeq \sum_{i} i \cdot n_{i}\left(\tau_{q}\right)
$$

The properties of the electron beam can all be described in terms of two potential differences within the EBIS, as seen in Fig. 1(b) (p. 12 ). The electron energy of the beam j.s retermined by $V_{e}$ and the electron current is determined by $V_{0}$ for the Pierce-type electron gun, with the ratio $V_{e} / V_{0}$ varying from 0.5 to 1.0 and $V_{0}$ varying from 1.0 to $10.0 \mathrm{kV}$ in the EBIS. Assuming that the electron gun has a constant perveance and that the electron beam is focussed by the magnetic field into a smooth Brillouin flow, one obtains an electxon beam of an approximately constant radius for these operating values of $V_{e}$ and $V_{0}$. Using a perveance of $2.0 \times 10^{-6}$ for the electron gun, and the Brillouin focussing field given by Eq. (2.14) for a beam radius of $0.5 \mathrm{~mm}$, yields for the electron beam in the EBIS the following parameters:

$$
\begin{aligned}
& E_{e}=e \cdot V_{e} \mathrm{keV} \\
& I_{e}=6.32 \times 10^{-2} V_{0}^{3 / 2} \mathrm{~A} \\
& \mathrm{~B}_{0}=0.743\left(V_{0}^{3} / V_{e}\right)^{1 / 4} \mathrm{KG} \\
& j_{e} \equiv I_{e} /\left(\pi r_{f}^{2}\right)=8.05 V_{0}^{3 / 2} \mathrm{~A} / \mathrm{cm}^{2} .
\end{aligned}
$$

Thus, using this description one finds that the electron density in the EBIS is

$$
n_{e} \simeq 2.74 \times 10^{10} V_{0}\left(V_{0} / V_{e}\right)^{1 / 2} \mathrm{~cm}^{-3}
$$

Since Eq. (2.7) gives the relative number of each charge state for the 
containment time ' $q$, one then can determine the number density for a given charge state $q$ after space-charge neutralization:

$$
q \cdot n_{q} \simeq \mathrm{f} \cdot n_{e}
$$

where $n_{e}$ is determined by Eq. (2.19) and $E \equiv q n_{q} /\left(\sum_{i} i n_{i}\right)$.

The fundamental lengths of interest in the FBIS are the mean free paths for elastic and inelastic collisions, Larmor precession radii in the magnetic field, and Debye length. The mean free path for elastic collisions is obtained by assuning binary coulomb collisions with a cutoff distance for scattering given by the Debye screening distance. Tinis assumption has been shown to be valid (Rose and Clark, 1967) and it makes the total coulomb cross section finite. Thus for Coulomb collisions between charged particles, the mean free path is given by

$$
\lambda_{c}=\frac{1}{n_{t} \cdot \sigma_{c}}
$$

where $n_{t}$ is the number density of target particles and $\sigma_{c}$ is the classical coulomb cross section with a cut-off scattering distance. This cross section is given by

$$
\sigma_{c}=\frac{\left(q_{1} q_{2}\right)^{2} e^{4} \ln \Lambda}{4 \pi \varepsilon_{0}\left(m v^{2}\right)^{2}}
$$

where

$$
\Lambda=\frac{12 \pi}{n^{1 / 2}}\left(\frac{\varepsilon_{0} k T}{e^{2}}\right)^{3 / 2}
$$

In Eqs. (2.22) and (2.23), $q_{1}$ and $q_{2}$ are the projectile and target charyes respectively, $\mathrm{m}^{2}$ is twice the relative kinetic energy of the particles, and $n$ and $T$ are the projectile number density and temperature respectively. For the EBIS, $2 n \Lambda \simeq 20$ and $\mathrm{m}_{e} v_{e}^{2}=3 k T e$, so that for electronion elastic collisions, using Eqs. (2.16) and (2.19) one obtains

$$
\lambda_{c} \simeq 6.1 \times 10^{7} \frac{V_{e}}{q^{2}}\left(\frac{V_{e}}{V_{0}}\right)^{3 / 2} \mathrm{~cm},
$$

where $q$ is the charge of the ion. 
For the irelastic collisions of the electrons with the ions, the total mean free path is given by $\lambda_{i}=\left(n_{0} \sigma_{t}\right)^{-1}$, where $\sigma_{t}$ is the total ionization cross section for the gas being ionized and $n_{0}$ is the initial number density of gas atoms. Using the approximation that $\sigma_{t} \simeq 10^{-16} \mathrm{~cm}^{2}$ for most gases used in the EBIS and $n_{0} \simeq 0.1 n_{e}$, yields

$$
\lambda_{i} \simeq \frac{3.6 \times 10^{4}}{V_{0}}\left(V_{e} / V_{0}\right)^{1 / 2} \mathrm{~cm} .
$$

The Debye screening length characterizes the screening effect of oppositely charged particles on the field of a charged particle and is derived and defined by spitzer (1956) as

$$
\lambda_{D} \equiv\left(\frac{k T_{e}}{4 \pi n_{e} e^{2}}\right)^{1 / 2} \mathrm{~cm} \text {. }
$$

For the EBIS one obtains, using Eqs. (2.16) and (2.19),

$$
\lambda_{D}=0.116\left(v_{e} / V_{0}\right)^{3 / 4} \mathrm{~cm}
$$

The last fundamental lengths used to describe the EBIS are the Larmor precession radil, given by $r_{L} \equiv m v_{\perp} /(e B)$, where $v_{\perp}$ is the velocity perpendicular to the magnetic field $B$. For the electrons, $r_{e L}=3.37 \times 10^{-3}$ $\times\left(E_{\perp}^{1 / 2} / B_{0}\right) \mathrm{cm}$; but $E_{\perp}=k T_{e \perp}$ eV and the transverse electron temperature nas been shown by Brewer (1959) to be given by $\left(r_{c} / r_{f}\right)^{2} T_{c}$, where $T_{c}$ is the cathooje temperature and $r_{c}$ and $r_{f}$ are the cathode and beam radii respectively. Since $T_{c} \simeq 11500^{\circ} \mathrm{K}$ in the EBIS, and the ratio $r_{e} / r_{f}=7$, one obtains for the electrons,

$$
r_{e \mathrm{~L}}=\frac{6.2 \times 10^{-3}}{\mathrm{~B}_{0}} \mathrm{~cm} \text {; }
$$

and similarly for the ions one obtains,

$$
r_{i_{L}}=0.145 \frac{A^{1 / 2} E_{\perp}^{1 / 2}}{q^{B}} \mathrm{~cm}
$$

where $q$ is the charge of the ion of mass $A$.

The Larmor radii are associated with one of the fundamental frequencies of the electron beam, the cyclotror frequencies which are defined 
as $w_{B} \equiv q B / m$, for, particle with charge $q$ and mass $m$ in a magnetic field B. Using $\nu_{B}=\omega_{B} / 2 \pi$, one obtains the frequencies for tine EBIS:

$$
v_{e B}=2.82 \times 10^{9} \mathrm{~B}_{0} \mathrm{~Hz} \text {, }
$$

and

$$
v_{i B}=1.54 \times 10^{6} q B_{0} / A \quad H z
$$

for tize electrons and ions respectively. The other fundamental frequency is the plasma frequency, defined as $\omega_{\mathrm{p}}=\left(4 \pi n q^{2} / A\right)^{1 / 2}$. For the electrons and ions in the EBIS, these plasma frequencies are

$$
v_{e p}=8.97 \times 10^{3} n_{e}^{1 / 2} \mathrm{~Hz}
$$

anđ̊

$$
v_{i p}=2.1 \frac{q}{\left(\text { An }_{i}\right)^{1 / 2}} \quad \mathrm{~Hz}
$$

respectively.

me ranges of values for all these quantities are shown in Table 1 for the design conditions within the Texas Adr EBIS. For the quantities involving ion charge and mass, $A^{+10}$ ions are assumed since pioduction of these ions is one of the design goals for this EBIS.

ilavirg calculated these Lasic stream parameters in the E'PIS, one can exanine them to determine the properties of the dynamics of the ion containment. The energies and censities in the EBIS indicate that it is a complex non-equilibrium stream. The mean free paths indicate that the electron-ion elastic collisions are much less important than the ionization collisions. The number densities are certainly large enough to make the collective effects of space charge and screening important. However, the Deoye length of the electrons is approximately equal to the dianeter of the beam, therefore a sheatn cannot form around the beam to shield it from the applied electric and magnetic fields. Thus the dynamics of containment can be examined microscopically for an individual ion witinin the electron beam.

- The effect of the magnetic fiela on the fundamental processes in tre plasma can be seen from the calculation of the Larmor radii. The electron radius of gyration $r_{e l}$ is seen to be muth smaller than the 
TABLE 1. Calculated parameters for the design conditions of the Texas A\&M EBIS.

\begin{tabular}{|c|c|c|c|}
\hline Quantity & Symbol & Range of values & Units \\
\hline Electron energy & $\ddot{E}_{e}$ & $0.5-10.0$ & kev \\
\hline Ion energy & $E_{i}$ & $1.0-10.0$ & $\mathrm{eV}$ \\
\hline Electron current & $I_{e}$ & $0.06-2.0$ & A \\
\hline $\begin{array}{l}\text { Electron current density } \\
\text { Electron beam potential }\end{array}$ & $j_{e}$ & $8.0-250.0$ & $\mathrm{~A} / \mathrm{cm}^{2}$ \\
\hline well depth & $\Delta V$ & $100.0-1000.0$ & $\mathrm{~V}$ \\
\hline Electron number density & $n_{e}$ & $3.0-40.0 \times 10^{10}$ & $e^{-/ / m^{3}}$ \\
\hline $\begin{array}{l}\text { Ion number density } \\
\text { Electron mean free path for }\end{array}$ & $n_{i}$ & $0.0-1.0 \times 10^{10}$ & ions $/ \mathrm{cm}^{3}$ \\
\hline $\begin{array}{l}\text { ion elastic collisions } \\
\text { Mean free path for ionization }\end{array}$ & $\lambda_{e}$ & $4.0-60.0 \times 10^{5}$ & $\mathrm{~cm}$ \\
\hline collisions & $\lambda_{i}$ & $4.0-25.0 \times 10^{3}$ & $\mathrm{~cm}$ \\
\hline Debye length & $\lambda_{\mathrm{D}}$ & $0.7-1.2$ & $\mathrm{~mm}$ \\
\hline Elactron Larmor radius & $r_{e L}$ & $2.0-8.0 \times 10^{-2}$ & $\mathrm{~mm}$ \\
\hline Ion Larmor radius & $r_{i L}$ & $1.25-2.0$ & $\mathrm{~mm}$ \\
\hline Electron cyclotron frequency & $v_{e B}$ & $2.0-8.0 \times 10^{9}$ & $\mathrm{~Hz}$ \\
\hline Ion cyclotron frequency & $v_{i_{B}}$ & $0.3-1.0 \times 10^{6}$ & $\mathrm{~Hz}$ \\
\hline Electron plasma frequency & $\nu_{e p}$ & $1.5-5.5 \times 10^{9}$ & $\mathrm{~Hz}$ \\
\hline Ion plasma frequency & $v_{i p}$ & $1.0-3.0 \times 10^{5}$ & $\mathrm{~Hz}$ \\
\hline Primary electron transit time & $\tau_{t}$ & $2.0-8.0 \times 10^{-10}$ & sec/cm \\
\hline Secondary electron drift time & $\tau_{\text {se }}$ & $0.5-8.0 \times 10^{-8}$ & $\mathrm{sec} / \mathrm{cm}$ \\
\hline $\begin{array}{l}\text { Transverse ion oscillation time } \\
\text { Mean time for electron ionization }\end{array}$ & $\tau_{\text {ose }}$ & $2.0-5.0 \times 10^{-7}$ & sec \\
\hline collision & $\tau_{i}$ & $2.0-6.0 \times 10^{-4}$ & sec \\
\hline Ion-ion self-collision time & $\tau_{i i}$ & $0.5-1.5 \times 10^{-6}$ & sec \\
\hline
\end{tabular}

electron beam radius and the Debye length. This means that the electrons have a small thermal rotation superimposed on the solid body rotation of the electron beam that occurs during injection into the magnetic 
field. The Larmor radii of the ions in the EBIS are seen to be greater than the Debye length or electron beam diameter. This important calculation shows that the effect of the magnetic field on the ions is negligible, only causing the ion orbits to precess slightly within the electron beam diameter. Thus the ions are magnetically free to fall through the potential drop of the electron beam and be electrostatically confined, much the same as they would be in an electrostatic well caused by ambipolar diffusion in a non-neutral plasma.

In order to study the confinement process further, the required containment time $\tau_{q}$ must be compared to otrer characteristic times involving the constituents in the EBIS. These are the electron and ion transit times in the beam, the mean collision times, and the energy relaxation times. For the electrons in the beam, the transit time in the EBIS, given by $\tau_{t}=1 / v_{e}$, is found to be

$$
\tau_{t}=0.55 \times 10^{-9} \mathrm{~V}^{\nu_{2}} \mathrm{sec} / \mathrm{cm} \text {. }
$$

Similarly, for the slow ejectrons created during ionization, the drift time is

$$
\tau_{s e}=1.7 \times 10^{-8} E_{e s}^{-1 / 2} \mathrm{sec} / \mathrm{cm},
$$

where $E_{e s}$ is the energy of the slow electrons in ev. Also of interest is tine transverse oscillation time of the ions in the potential of the electron beam given approximately by

$$
\tau_{\text {OSe }}=1 / \nu_{\text {OSe }}=0.45 \times 10^{-6} r_{f}\left(\frac{A}{Q \Delta V}\right)^{\frac{1}{2}} \mathrm{sec},
$$

where $\Delta V$ is the effective space-charge potential depth in $V$ for the ions of atomic mass $\bar{A}$ and charge $q$ (Jain and Divatia, 1975).

The mean collision times are related to the mean free paths given earlier $b_{y}$ the relationship $\tau_{C}=\lambda_{C} / v$. The e'ectron ionization mean collision time is then

$$
\tau_{i}=1.9 \times 10^{-4} V_{0}^{1 / 2} \mathrm{sec} \text {. }
$$

The values given in Table 1 (p. 34$)$ for these transit times and mean collision time indicate that the electrons in the beam suffer very few collisions in their passage througin the EBIS. Also, the ions are seen 
to execute many oscillations between ionizing collisions. The slow secondary electrons will also diffuse axially along the EBIS to the grounded collector much faster than they are produced by ionization collisions if the reflection of these electrons by the ion extractor voltage is negligible. However, if these electrons are reflected, it is also possible for them to gain energy very rapidly by collisions with the other electrons .

The energy exchange between identical paricles due to coulomb collisions, as shown by spitzer (1956), has a "self-collision time" given by

$$
\tau_{s c}=\frac{11.4 \mathrm{~A}^{1 / 2} T^{3 / 2}}{n q^{4} \ln \Lambda} \quad \sec .
$$

Using the EBIS parameters, one obtains for the electrons

$$
\tau_{e e}=1.66 \times 10^{4} \frac{\left(k T_{e s}\right)^{3 / 2}}{n_{e s}} \mathrm{sec},
$$

where $n_{e s}$ is the number density of slow electrons in $\mathrm{cm}^{-3}$ and $k T$ es is their mean temperature in ev. For the ions one obtains

$$
\tau_{i i}=7.12 \times 10^{5} \frac{\mathrm{A}^{1 / 2}(k T)^{3 / 2}}{n_{q} q^{4}} \mathrm{sec} .
$$

Thus, Eq. (2.37) indicates that those slow electrons which do not escape are heated very rapidly by collisions with the beam electrons, once their density reaches only a small fraction of the electron beam density. That such a process occurs for these secondary electrons is borne out in the fact that the simple successive ionization theory without ion loss mechanisms, the largest of which would be ion-electron recombination with the secondary electrons, fits the experimentally measured charge state distributions (Donets and Pikin, 1975).

For the ion-ion collisions, the self-collision time gives a measure of how quickly a Maxwellian distribution of ion energies is formed. To evaluate this time in the EBIS one uses $n_{q}=f n_{e} / q$ and obtains 


$$
\tau_{i i}=2.6 \times 10^{-5}\left(\frac{V_{e}}{V_{0}}\right)^{1 / 2} \frac{\AA^{1 / 2} T_{q}^{3 / 2}(\mathrm{eV})}{f q^{3} V_{0}} \mathrm{sec} .
$$

As shown in Table 1 (p. 34) this yields an ion energy relaxation time that is much shorter than the ion contaimment time for all ions being prouluced in the EBIS, particularly if the ion temperature remains low,

Examining the ion heating and cooling processes during ion confinement, one finds that the ions gain their energy from the potential energy of their position at ionization within the electrostatic potential of the electron beam and also from electron-ion elastic collisions. But the electron-ion elastic collisions impart energy to the ions at a very slow rate, given by Jancel and Kahan (1966) as

$$
\left[\frac{d E}{d t}\right)_{e \rightarrow i}=2.8 \times 10^{-9} \frac{q^{2} n_{e}}{A \cdot V_{e}^{1 / 2}} \mathrm{eV} / \mathrm{sec},
$$

or, for the EBIS

$$
\left(\frac{d E}{d t}\right)_{e \rightarrow i}=77.0 \frac{q^{2} V_{0}^{3 / 2}}{A \cdot V} V_{e} \mathrm{eV} / \mathrm{sec} .
$$

For the case of $A^{+10}$, this rate is only a few hundred eV/sec. In the stripping process, at each ionization the potential energy of the ions changes by $e \phi$, where $\phi$ is the potential at the ionization position. The total energy then acquired by the ion during ionization to charge state $q$ is given by

$$
E_{i}=e \sum_{i=1}^{q} \phi\left(r_{i}\right)
$$

where $\phi\left(r_{i}\right)$ is the electrostatic potential at the $i$ th ionization position $r_{i}$. Janes et $a l$. (1966) have shown that for an ion oscillating very rapidly in a potential well with a constant depth $\Delta \mathrm{u}_{0}$ the ion energy acquired during ionization to charge state $q$ can be calculated statistically by

$$
E_{i} \simeq q \cdot e \cdot E_{i},
$$

where 


$$
r_{i} \simeq 1.1 \Delta \mathrm{u}_{0} q^{-1 / 3}
$$

In the EBIS $\Delta \mathrm{U}_{\mathrm{u}}$ is not a constant since space-charge neutralization is occurring as the ionization takes place. This further reduces the energy the ions acquire during ionization.

In addition, the ions are subject to a cooling process since energetic ions that escape the decreasing potential well lower the average energy of the ions remaining in the well. Iinder and Hernquist (1950) have shown that even at space-charge neutralization, there may still be a potential depression of a few volts in the electron beam which traps the ions. This would indicate that the ion energy must be less than approximately one eV per charge. This happens because the potential well at space-charge neutralization arises from the unnentralized charge, $n_{e}^{-n^{+}}$, but since these are both large rumbers, $n_{e}^{-n^{+}}$may be large enough to produce a small potential depression even though $n_{e} / n^{+}$is approximately unity. Here $n^{+}$is the total density of positive ions.

In order to numerically determine the ion energy, the microscopic parameters for an ion are assumed to be average values for an ensemble of ions describable by a set of fluid equations. Such an approach has been taken by Wieseman (1972) for ions electrostatically trapped in a potential due to ambipolar diffusion in a charged plasma containing energetic electrons. He assumes that energy relaxation between the ions occurs very rapidly, as in the EBIS. Following his hydrodynamical procedure for representing the energy balance of the ions in the electrostatic well, and assuming his approximations as being applicable to the EBIS, one writes his result for the EBIS as

$$
k T_{i} \simeq 5.5 \times 10^{-4} k T e \frac{q^{2}}{A}+k T g .
$$

But in the notation that has been used in this work, $k T e^{=2 e V} e^{/ 3}$, and $k T \simeq 0.04 \mathrm{eV}$, so the ion energy in the EBIS is

$$
k T_{i} \simeq 0.37 V_{e} \frac{q^{2}}{A}+0.04 \mathrm{eV}
$$

One of the observable consequences of the ion temperature is the emittance of the ions extracted from the ion source. The emittance of 
an ion beam is defined as the area of a two dimensional phase space occupied by the particles in the beam. For a beam with axial symmetry, eacn particle has a radial position $r$ and a radial divergence $r^{\prime} \equiv d r / d z$. 'The area in $r-r$ ' phase space occupied by all the particles in an ion beam is tile emittance of that ion beam. This area is usually approximated by an ellipse of uniform density with area $x_{0} x_{0}^{\prime}$, where $x_{v}^{\prime}$ and $r_{0}$ are the maximua values. Since $r_{0}^{\prime}$ depends on the axial velocity (beam energy), the emittance is energy dependent. This dependence is remuved by defining a normalized emittance. The normalized emittance is defined as

$$
\varepsilon=\pi r_{0} r_{0}^{\prime} B
$$

where $r_{0}$ and $r_{0}^{\prime}$ are as defined as above; and $\beta \equiv v / c$, where $v$ is the ion axial velocity and $c$ is the velocity of light. But $r^{\prime} \beta=\dot{r}_{c}$, where $\dot{r} \equiv v_{\perp}=\left(2 E_{\perp} / \mathrm{m}\right)^{1 / 2}=\left(27 T_{i} / \mathrm{m}\right)^{1 / 2}$. Thus the ion temperature is a direct measure of the radial velocity of the ions trapped in the electron beam. Using the ion temperature given in Eq. (2.45), one can define the EBIS normalized enittance as

$$
E=0.146 r\left(\frac{0.37 q^{2}}{A^{2}} V_{e}+\frac{0.04}{A}\right)^{1 / 2} \mathrm{mm-mrad} .
$$

If one assumes that the extraction radius is $\simeq 2 \mathrm{~mm}$, and solves this equation for $\mathrm{Kr}^{+8}$ at $V_{e}=10 \mathrm{kV}$, one obtains $\mathrm{E}=0.054 \times 10^{-6} \mathrm{~m}-\mathrm{rad}$. The value of emittance calculated by Ton That (1972) for this beam using a simulated particle computer code is $\varepsilon=0.056 \times 10^{-6} \mathrm{~m}-\mathrm{rad}$, and an emittance calculated by Becker, Klein, and Schmidt (1972) for $U^{+25}$ ions at an electron energy of $2.5 \mathrm{keV}$ yielded $\varepsilon=0.042 \times 10^{-6} \mathrm{~m}-\mathrm{rad}$. Thus, as stated earlier, the Texas A\&M EBIS is expected to produce ion beams of small emittances. 
EXPERIMENTAL APPARATUS

A. General Description

A cutaway view of the prototype EBIS constructed at Texas A\&M is shown in Fig. 6. This model uses a one meter long solenoid for focussing the electron beam within the ionization region. The electron gun is placed in a vacuum chamber external to the solenoid bore, and shielded from the magnetic field by a pole piece used as one face of the cubical vacuum chanber. This vacuim chamber is pumped by an electrostatic getter-ion pump. The vacuum chamber on the other end of the solenoid contains the electron collector-ion extractor assembly, electrostatic focussing lenses, and the feed-throughs to the ionization region. The vacuum in this chamber is produced by a liquid nitrogen cooled titanium sublimation pump in parallel with a turbomolecular pump. Both chambers are isolated from the hot filament pumps by large stainless steel gate valves which allow pump cleaning and filament replacement without venting the vacuum system, while keeping the conductance to the punps very high. The ionization chamber located within the bore of the solenoid contains the cylindrical electrodes through which the electron beam is focussed. This system of electrodes (drift tubes) is located within a cylindrical liquid nitrogen dewar that also contains a liquid helium cryopumping surface, and a shielded line for injecting the working gas. The twenty drift tubes are each mounted independently on two insulators to allow precise alignment of the assembly along the axis of the solensid, and they are vacuum gapped to allow pumping of the region within the drift tubes by the cryosurface. The insulators are also used to support the voltage leads necessary for application of the axial potentials to the electrodes.

After this prototype had been constructed, the one meter solenoid was found to have magnetic field inhomogeneities that were not correctable, so the solenoid was disassembled and a shorter magnet was constructed from some of the useable coils. A new ionization chamber was constructed for this short solenoid to test the electron beam focussing 


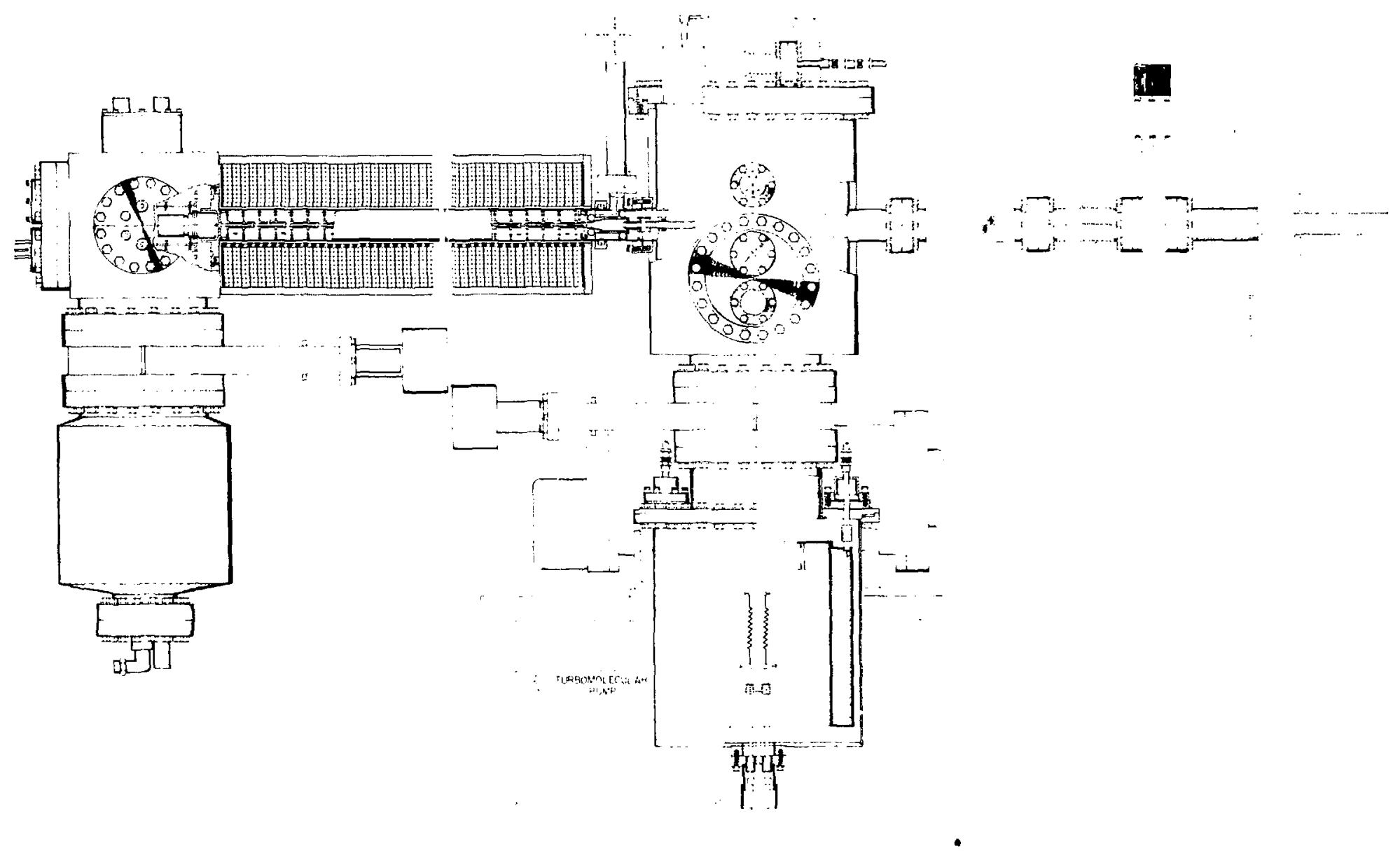

FIG. 6. Cutaway view of the Texas AsM prototype EBIS. 
system, and to study the ionization processes as was to be done with the prototype. The results obtained from this test model, as well as the preliminary results from the prototype, can be used to establish the design of an EBIS for use with the Texas A\&M cyclotron.

The ionization region of the short test EBIS is shown in Fig. 7. The six drift tubes are mounted on an assembly that slides into the vacuum chamber, and a liquid nitrogen cryosurface runs through the vacuum chamber. This test EBIS uses a $20 \mathrm{~cm}$ magnet for electron beam focussing, and only has liquid nitrogen cryopuming within the ionization chamber. However, the rest of the device is the same as that for the prototype described above.

In both cases, the complete ion source system is electrically grounded and connected via a vacuum insulator to a magnetic analysis system, consisting of a $\mathrm{C}$-magnet with an isolated high voltage vacuum chamber. This chamber contains current measuring instrumentation inductively ccupled to a grounded recording system.

B. Vacuum System

The main components of the ERIS vacuum system are shown schematically in Fig. 8. The chief material used in fabrication of the system was type 304 stainless steel. Stainless steel flanges with copper gaskets were used for most of the demountable seals, with Viton "O"-rings used on the few seals which did not permit copper gaskets because of structural limitations. Standard ultrahigh vacuum (uhv) techniques in welding and cleaning of all parts were practiced (Guthrie, 1963; Wheeler, 1972). Except for the vacuum insulator to the high voltage analysis system, no glass was used in order to avoid a large helium background by diffusion from the atmosphere. The entire vacuum system was designed for bake-out at $200^{\circ} \mathrm{C}$.

Since the ultimate pressure obtainable in any vacuum system represents an equilibrium between the rate of gas production in the system and the rate of gas removal from the system, the gas removal should be maximized and the gas production should by minimized. The uhv materials and techniques described above served to minimize the gas production due to outgassing and permeation froin the system components. The vacuum 


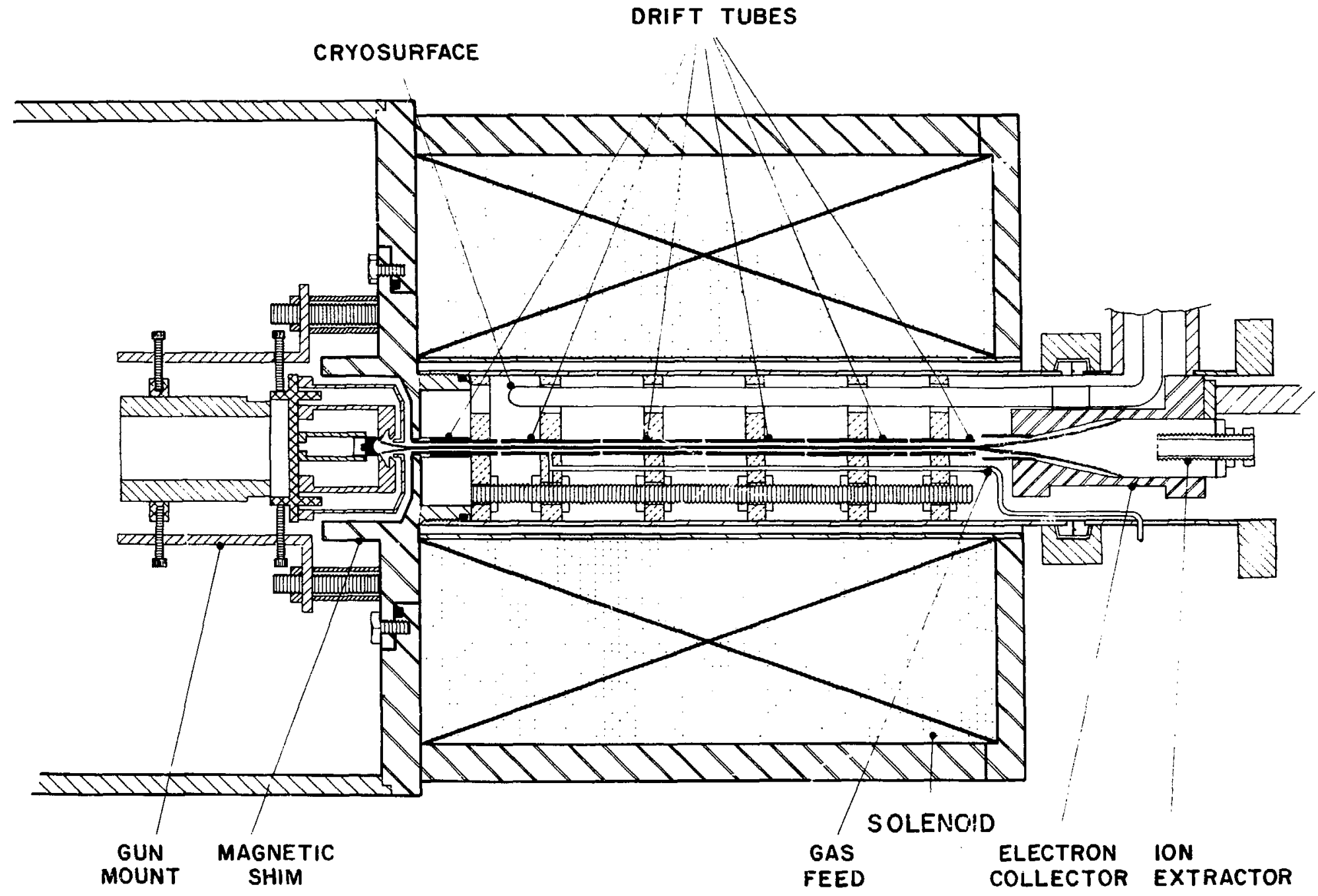

FIG. 7. Cutaway view of the ionization region of the short test EBIS. 


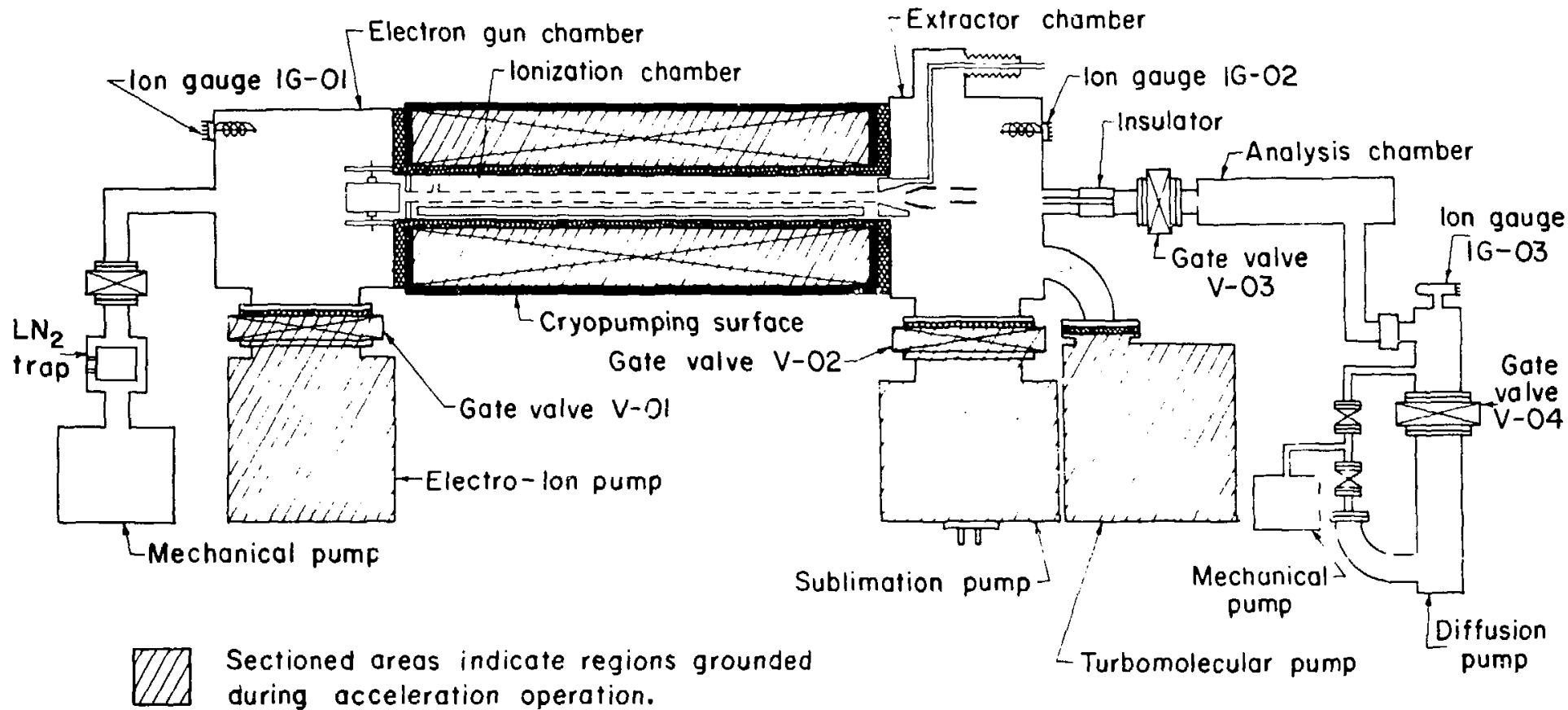
during acceleration operation.

Cross-hatch indicates isolation flanges $\&$ liners necessary for accelerator operation.

FIG. 8. Vacuum system of the Texas A\&M EBIS. 
pumps were selected in order to maximize the gas removal, with simplicity of operation and the EBIS vacuum requirements as prerequisites in the choices.

Pumping during bake-out and stand-by operation is supplied only by a Weich turbomolecular pump (260 liters/sec for air). During source operation a liquid nitrogen cooled titanium sublimation pump $(15,000$ liters/sec for air) is operated in parallel with the turbomolecular pump on the extractor chamber. Such a combination has been shown to attain vacuums of $10^{-11}$ Torr (Henning, 1972). Pumping of the electron gun chamker is carried out with a 10 inch Granville Phillips Electro-Ion jump (1400 liters/sec for air), with roughing carried out by a mechanical pump and a liquid nitrogen trap-valve. The analysis system is pumped by a 4 inch NRC VHS diffusion pump, with a liquid nitrogen chevron baffle (1200 liters/sec for air). In the one meter prototype, once the ionization chamber has been pumped to a good vacuum with the pumps on each end of it, the ultrahigh vacuum within it is produced by the cryopumping of a liquid helium cooled surface (1000 liters/sec for air), and the liquid nitrogen dewar surrounding the cryosurface. The cryopumping in the short test model is provided only by a liquid nitrogen cryosurface at this point, causing the residual pressure to be at least an order of magnitude greater in the ionization region. However, tre ionization region in the test model is not so conductance limited for pumping by the extractor chamber pumping system, so this increase is reduced somewhat by the larger conauctance.

Pressure calculations have been carried out for the vacuum system of the prototype source. A lumped conductance approach was used for representing the complicated pumping geometry as shown in Fig. 9. The flow equations for this system are then given by:

$$
\begin{aligned}
& P_{1} \simeq Q_{1} / S_{e_{1}} \\
& P_{2} \simeq Q_{2}+C_{8}\left(P_{1}-P_{2}\right)+C_{7}\left(P_{3}-P_{2}\right) / S_{e_{2}} \\
& P_{3} \simeq Q_{3}+C_{6}\left(P_{4}-P_{3}\right) /\left(S_{e_{3}}+S_{\epsilon}\right) \\
& P_{4} \simeq Q_{4}-C_{6}\left(P_{4}-P_{3}\right) / S_{e_{4}}
\end{aligned}
$$




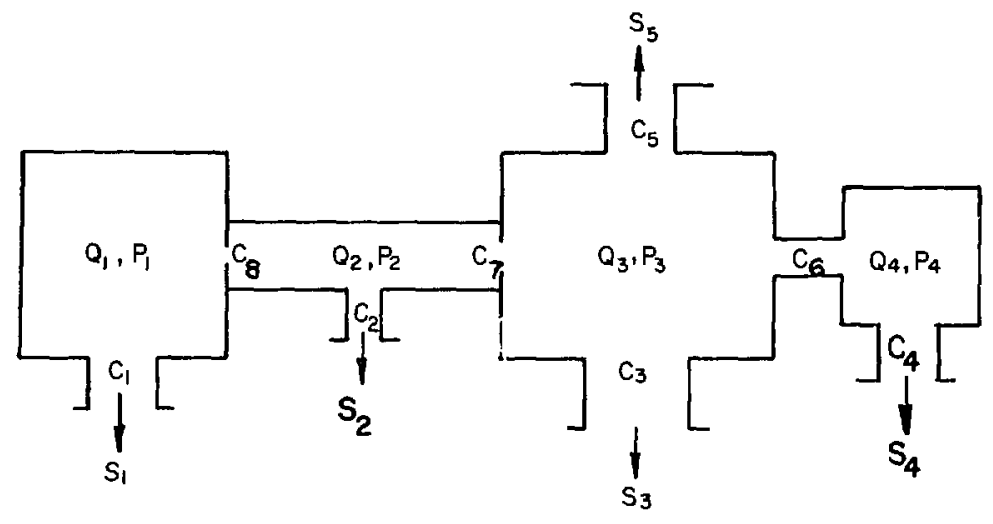

FIG. 9. Lumped conductance repitentation of the EBIS vacuum system used for pressure cal.culations.

where $s_{e_{i}}=C_{i} s_{i} /\left(C_{i}+s_{i}\right)$ is the effective pumping speed of the $i$ th pump. The variables in these equations, as represented in Fig. 9 above, are defined and evaluated as follow's:

a) $Q_{1}$ is the gas load of the electron gun chamber due to wall and electron gun outgassing; $6.0 \times 10^{-7}$ Torr-liters/sec.

b) $C_{1}$ is the combined conductance of the electron yun chamber flange and gate valve; 2030 liters/sec.

c) $s_{1}$ is the pumping speed of the electrostatic sputter pump; 1400 liters/sec.

d) $Q_{2}$ is the gas load in the ionization chamber due to outgassing; $3.5 \times 10^{-9}$ Torr-liters/sec.

e) $\mathrm{C}_{2}$ is the effective conductance of the drift tubes; 125 liters/ sec.

f) $S_{2}$ is the local pumping speed of the cryosurface of total area $400 \mathrm{~cm}^{2}$. Since only half of its surface is exposed to the gas load, this effective pumping speed is taken to be only half of its total pumping speed for the entire surface; 10001 iters/sec. 
g) $Q_{3}$ is the gas load due to outgassing in the extractor chamber; $8.0 \times 10^{-8}$ Torr-liters/sec.

h) $C_{3}$ is the combined conductance of the extractor chamber flange, gate valve, and titanium pump baffle; 950 liters/sec.

i) $C_{5}$ is the combined conductance of the elbow and bellows on the turbomolecular pump; 380 liters/sec.

j) $S_{3}$ is the pumping speed of the titanium sublimation pump; 5000 liters/sec.

k) $S_{5}$ is the pumping speed of the turbonolecular pump; 260 liters liters/sec.

1) $Q_{4}$ is the gas load in the analysis chamber due to outgassing; $1.2 \times 10^{-7}$ Torr-liters/sec.

m) $\mathrm{C}_{4}$ is the combined conductance of the pump line and manifold on the 4 inch diffusion pump used on the analysis system; 10 liters/sec.

n) $\mathrm{S}_{4}$ is the pumping speed of the 4 inch diffusion pump with the cold trap and valve on it; 850 liters/sec.

o) $C_{6}$ is the combined concuctance between the analysis chamber and extractor chativer; 6.5 liters/sec.

p) $c_{7}$ is the effective conductance between the extractor chamber and ionization chamber; 9 liters/sec.

q) $C_{\theta}$ is the effective conductance between the ionization chamber and the electron gun chanber; 0.1 liters/sec.

Using these values to solve Eqs. (3.1), the calculated pressures are yiven as follows: $P_{1}=7 \times 10^{-10}$ Torr, $P_{2}=4 \times 10^{-11}$ Torr, $P_{3}=1.3 \times 10^{-10}$ Torr, and $P_{4}=7 \times 10^{-9}$ Torr. A similar calculation for the $20 \mathrm{~cm}$ EBIs yields approximately the same results with $\mathrm{F}_{2}=5 \times 10^{-10}$ Torr being the only significant change.

Another calculation of interest is the lifetime of the liquid helium cryosurface in the prototype EBIS. Assuming a gas load of $10^{-6}$ Torr-liters/sec for the ionization chamber during operation yields a lifetime of about 400 hours, at a consumption rate of about 0.75 liters per hour. The lifetime of the cryosurface is here assumed to be covexage of the surface by 200 monnlayexs of gas. This consumption rate does 
not cover the helium loss in the transfer tube to the system, but only the losses due to gas adsoxption, radjation heating, and heat conduction of the helium lines within the vacuum system of the EBIS. The transfer tube, a standard vacuum-insulated transfer line with a $1 / 8$ in. transfer line inside a $3 / 4$ in. vacuum jacket, should have a loss rate of about the same magnitude as the cryosurface, yielding a total helium consumption of about 1.5 liters per hour. This rate, high but not intolerable, is necessary to obtain the residual vacuun needed in the rather inaccessible ionization region. Much of the cost however, could be reduced by a recovery system for the gas, or by use of a helium refrigerator.

c. Electron Beam System

The electron beam is formed in the electron gun chamber by the Pierce-type electron gun, injected into and focussed through the ionization region by the magnetic field of the solenoid, and stopped by a grounded collector at the opposite end of the ionization chamber in the entrance to the extractor chamber. As stated earlier, the stability of the electron beam in the EBIS is maintained by Brillouin focussing, so the electron gun and focussing system were designed for this type of electron flow.

The electron gun chosen for use in the EBIS is a modified Piercetype convergent gun of the type widely used in the traveling-wave tubes built by the electronics industry. This 112-2B electron gun has a perveance of $2.0 \times 10^{-6} \mathrm{~A} / \mathrm{V}^{3 / 2}$ and an electrostatic beam areal convergence of about 36. It is designed to operate from one to ten $\mathrm{kV}$, with a nominal operating voltage of $6 \mathrm{kV}$. Operating characteristics for this electron gun as measured by the manufacturer are given in Table 2 .

The $112-2 B$ electron gun is a scaled version of the $112-2 \mathrm{~A}$ test gun, a demountable electron gun with a perveance of $2.22 \times 10^{-6} \mathrm{~A} / \mathrm{V}^{3 / 2}$. The scaling was calculated to yield an electrostatically focussed beam of $1.0 \mathrm{~mm}$ diameter at $6 \mathrm{kV}$, but in order to make the cathode of the gun replaceable with the alignment accuracy required, an alignment hole was drilled in the center of the cathode causing a slightly larger beam.

${ }^{3}$ Built for the Texas A\&M Cyclotron Institute by Hughes Aircraft Company, Electron Dynamics Division, Torrance, California. 
TABLE 2. Operating characteristics of the $112-2 B$ electron gun.

\begin{tabular}{|c|c|c|c|c|}
\hline Voltage $(\mathrm{kV})$ & 2 & 4 & 6 & 7 \\
\hline Beam Current (A) & 0.192 & 0.515 & 0.918 & 1.17 \\
\hline Anode Current (A) & 0.0025 & 0.0064 & 0.0115 & 0.0147 \\
\hline Perveance $\left(A / V^{3 / 2}\right)$ & $2.15 \times 10^{-6}$ & $2.04 \times 10^{-6}$ & $1.98 \times 10^{-6}$ & $2.00 \times 10^{-6}$ \\
\hline Minimum Bean Radius (mm) & 0.74 & 0.69 & 0.67 & 0.65 \\
\hline Minimum Beam Distance & & & & \\
\hline from Anode (mm) & 3.8 & 4.0 & 4.1 & 4.1 \\
\hline \multicolumn{5}{|l|}{ Minimum Current } \\
\hline Density $\left(\mathrm{A} / \mathrm{cm}^{2}\right)$ & 24 & 85 & 180 & 261 \\
\hline
\end{tabular}

This small hole also caused the beam density to be more sharply peaked than the beam from the uniform cathode, much the same as the beam from a cathode with a hole in it caused by ion bombardment (Cutler and saloom, 1955). However, the electron beam emitted by the 112-2B gun is still very laminar in its flow.

The demountable structure of the electrodes in this gun is primarily for experimental testing of electron gun designs, but has been very useful for the experimental work on the development of the EBIS. For the 112-2B gun used in this work, the cathodes have been poisoned several times by bad vacuum conditions, and the anode and focus electrode have been coated by material sputtered with the electron beam. Thus all the electrodes have been removed for cleaning or replacement several times, and no deterioration in beam quality or gun performance has resulted.

The mounting method for the electron gun in the EBIS and the magnetic shim used to shield the cathode from the magnetic field of the solenoid and cutain the proper entrance condition for injection of the beam into the magnet are shown in Fig. 10. The shim is fabricated from lowcarbon iron and is nickel plated to reduce outgassing in the ultrahigh vacuum of the electron gun chamber. The wall of the vacuum chamber on which the shim is mountedis also nickel-plated mild steel, so that the 


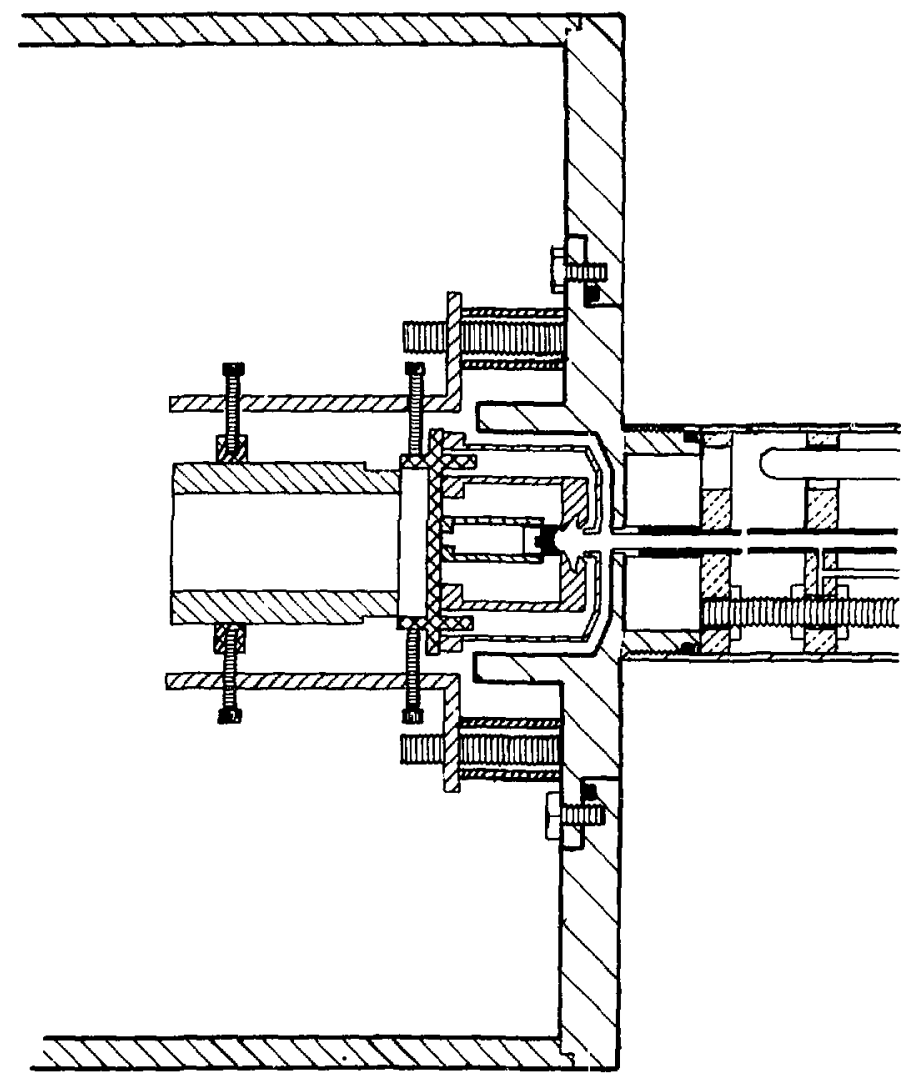

FIG. 10. Electron gun mounting and magnetic shielding configuration. 
shim and chamber wall are part of the flux return sheath enclosing the solenoid. This mild steel chamber wall was heli-arc welded to the other walls, all made of type 304 stainless steel, and then nickel-plated. The magnetic shim has a $5.6 \mathrm{~mm}$ diameter hole of thickness $3.3 \mathrm{~mm}$, and the electron beam is focussed througin this hole into the drift tubes along the axis of the solenoid. The results of Molnar and Moster (1951) show that for these entrance conditions, the electron beam has a diameter of approximately $1 \mathrm{~mm}$ within the drift tubes, whose inside diameter is $3 \mathrm{~mm}$.

The electron beam is maintained at this focussed diameter through the drift tubes by the uniform magnetic field of the solenoid. The magnetic field axis must be aligned very precisely with respect to the electron deam axis for complete transmission of the beam through tis solenoid. Deviation of only a few milliradians from the optimum alignment has been shown to affect the beam transmission very drastically (Nishihara and Terada, 1968). To allow for alignment of the magnetic axis and electron beam axis in the EBIS, the soleroid was constructed with a $21 / 4$ in. inside diameter, while the ionization vacuum chamber was constructed with a 2 in. outside diameter. The solenoid originally built for the prototype ion source was $z$ one meter long, air core, water-cooled magnet capable of producing an axial magnetic field of $9 \mathrm{kG}$ at a current of 500 A. The magnetic axis of this solenoid was measured and found to have local deviations along the length of the magnet greater than $10 \mathrm{mrad}$. In addition, it had a large linear tilt with respect to the axis of the bore, with a curvature on each end. First order correcuions could be made to aligr tive magnetic axis with the electron beam axis by tilting the solenoid with respect to the ionization chamber and off-setting the aperture in the shim on each end of the solenoid, but deviations of more than 5 mrad still existed.

This solenoid was disassembled and some of the double wound pancake coils were used, along with a piece of the original inner bore tube and outer flux sheath, to construct a shorter version of this magnet. The shorter solenoid has a length of $20 \mathrm{~cm}$ and consists of 12 double wound pancake coils with 10 turns of wire in each layer. This magnet produces 
an axial field of $7.5 \mathrm{kG}$ with a current of $500 \mathrm{~A}$. The magnetic axis still has a linear tilt, most probably due to the pitci of the large hollow windings, but the local deviations of the magnetic axis about this tilted axis are less than 2 mrad. The axial majnetic field for this solenoid, with the shims in position on each end is shown in Fig. 11 (a). The field build-up through the small hole in the shim on the electron gun chamber is shown in expanded view in Fig. Il(b), along with the theoretical description of this build-up as given by Eq. (2.15).

As the electron beam leaves the solenoid, it begins to blow up in the fringing field, and is collected by a water-cooled, grounded electron collector. An extensive theoretical study by Ton that (1972) has established a desired shape for such an electron collector, and the beam envelope calculations of Bevec, Palmer, and susskind (1958) were used to obtain the dimensions of the electron collector for the electron beam in the Texas A\&M EEIS. The position of the electron collector and the calculated beam envelope are shown in Fig. 7 (p.45) for the test EBIS.

Computer calculations have also been made for the electron beam system to study the electron gun and the injection of the electron beam into the magnetic field of the solenoid. This computer simulation has been done with a modified version of the electromagnetic focussing program EMGUN by Boers (1968). The simulation of the 112-2B gun is shown in Fig. 12 for an operating voltage of $2 \mathrm{kV}$. The calculated perveance is $2.15 \mathrm{x}$ $10^{-6} \mathrm{~A} / \mathrm{V}^{3 / 2}$, exactly the measured value, and the calculated beam minimum occurs $3.9 \mathrm{~mm}$ in front of the anode face, which is only $0.1 \mathrm{~mm}$ greater than the measured position of the minimum. The experimental current density measured by the electron gun manufacturer and the computed current density at the beam minimum are shown in Fig. 13. The agreement is rather good, the difference being due to the fact that thermal effects are içnored in the computer calculation.

For the electron beam injection calculations, the geometry in Fig. 10 (p. 52) for the relative electron gun, drift tube and magnetic shim positions was assumed, along with the measured magnetic field shown in Fig. $11(\mathrm{~b})$ (p. 56 ), and the magnitude of the magnetic field was varied until a smooth focussed beam was obtained. Such a calculation for the electron gun operating at $6 \mathrm{kV}$ is shown in Fig. 14. The focussed beam 

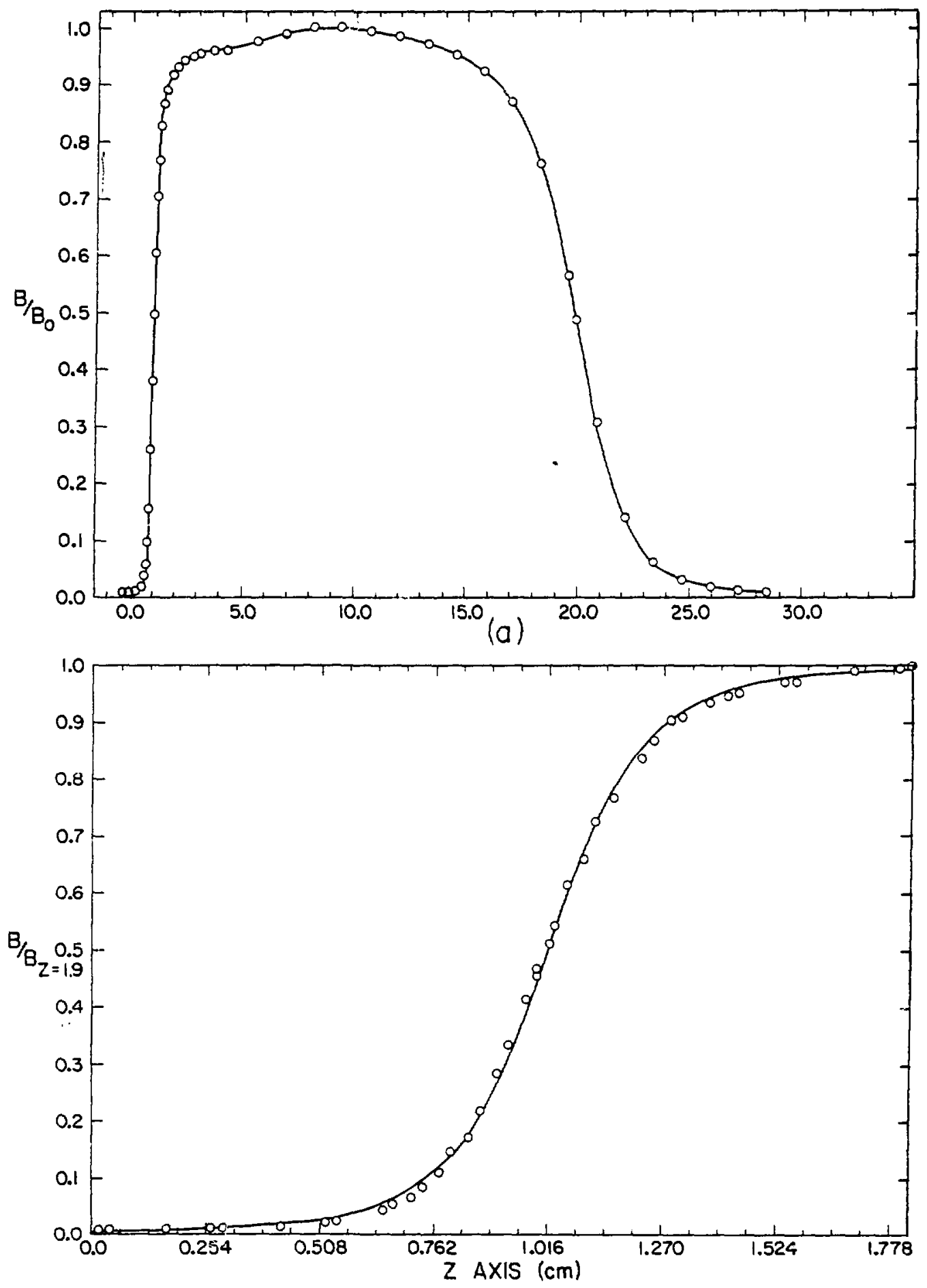

(b)

FIG. 11. (a) Axial magnetic field in the $20 \mathrm{~cm}$ test solenoid; (b) Expanded view of the axial magnetic field through the electron gun magnetic shim. 


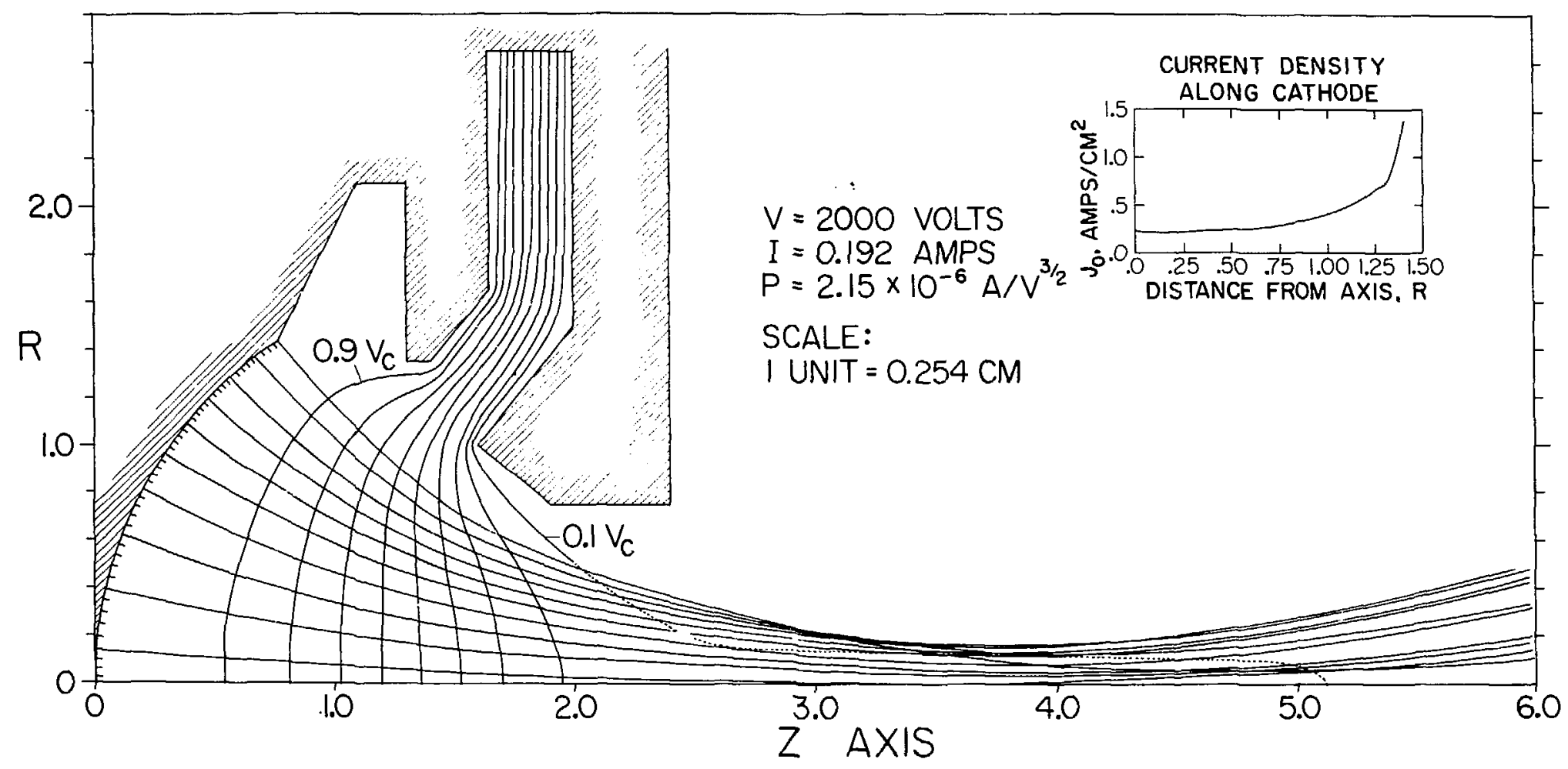

FIG. 12. Computer prediction for the $112-2 B$ electron gun electron trajectories using the axisymmetric finite difference space charge code EMGUN (Boers, 1968). Also shown is the predicted cathode current density and the space-charge compensated equipotentials. 


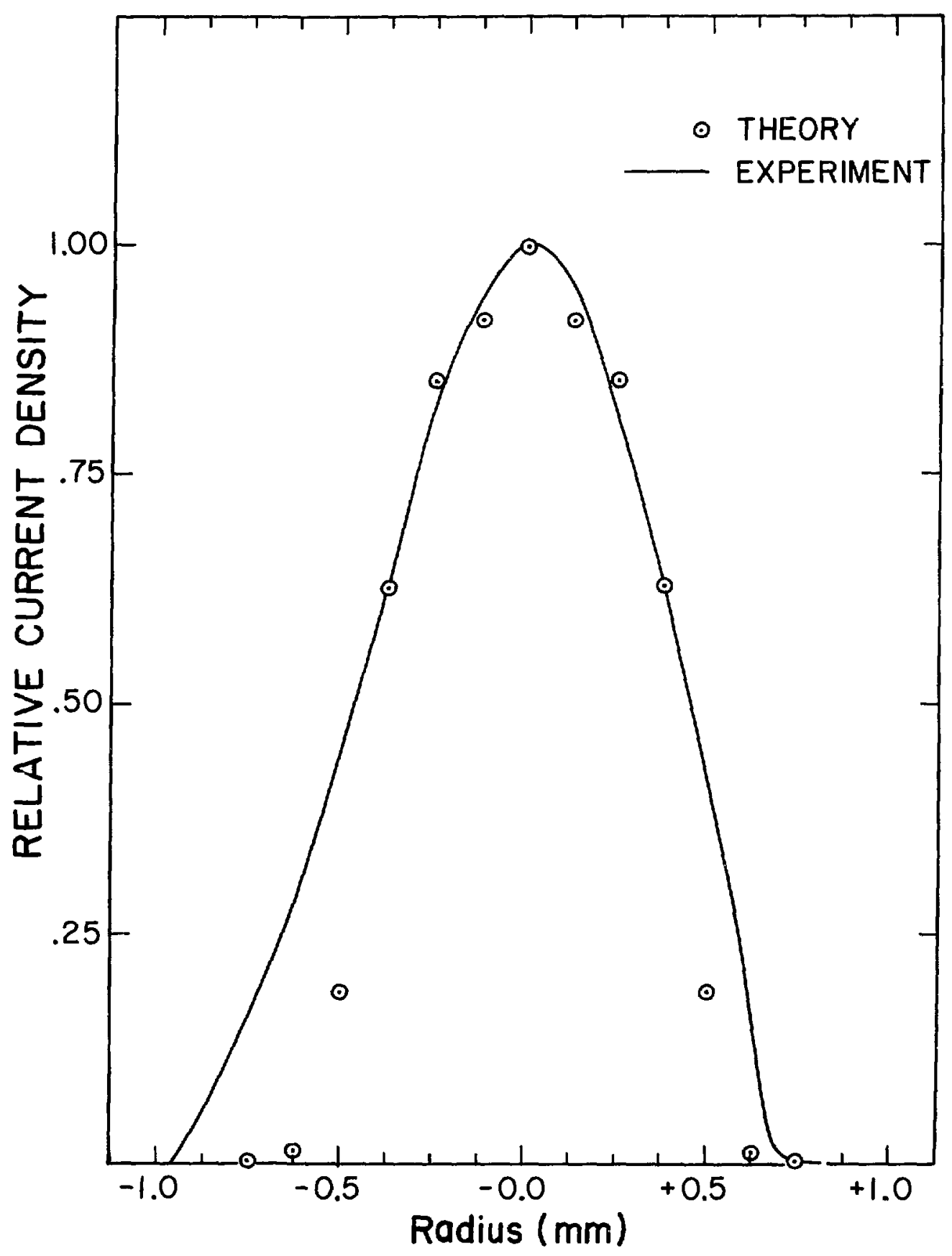

FIG. 13. Comparison of the measured electron beam current density at the beam minimum with the calculated current density for an electron energy of $2 \mathrm{keV}$ with no nagnetic focussing. 


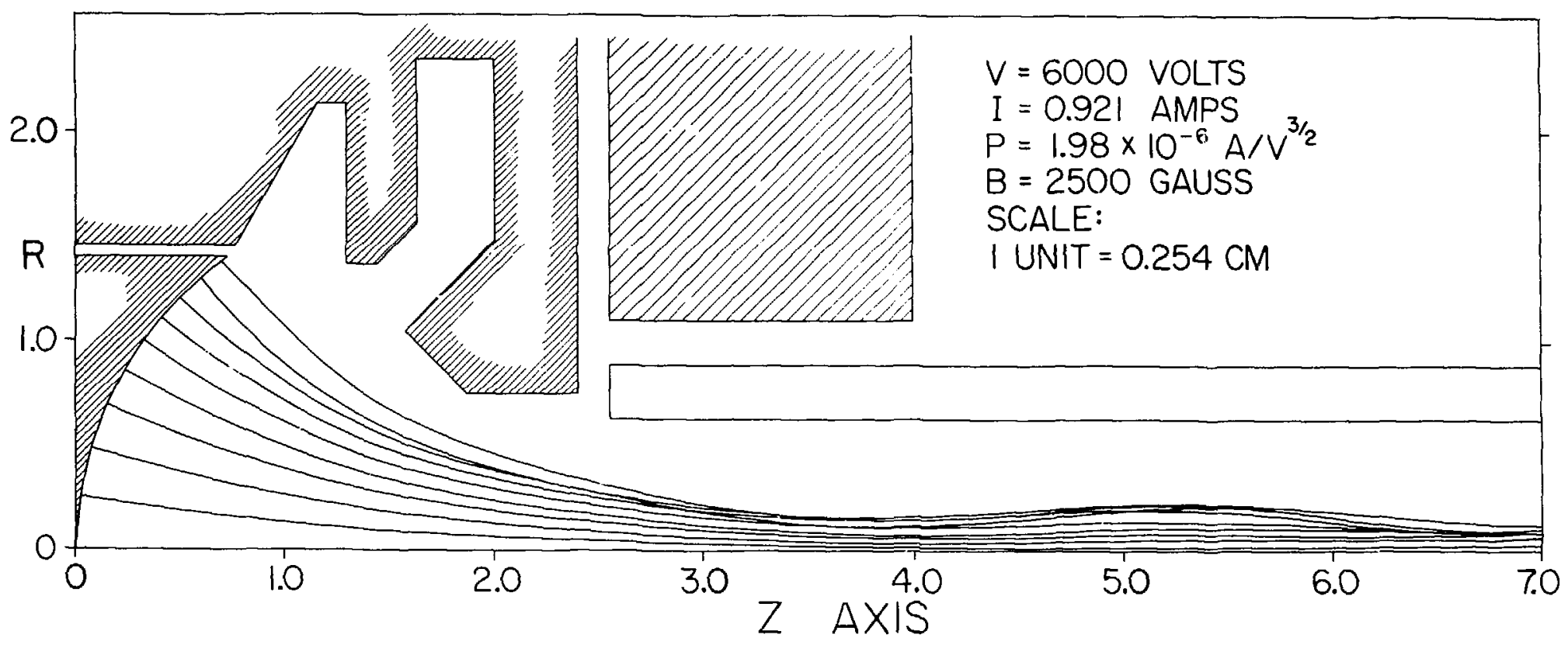

FIG. 14. Calculated focussing of the electron beam in the EBIS for an electron energy of $6 \mathrm{keV}$. 
has a slight ripple in it and the focussing ficld is larger than the Brillouin value, but these are due to a small flux threading the cathode. This flux can be eliminated in future work by an axial compensating coil behind or around the cathode. 2.lso, the calculated beam in all cases has a small translaminar stream that is not prominent in the experimenially measured beam. This stream, and a large current loading on the edge of the cathode are present in all of the calculations. These problems are probably due to the large mesh spacings necessary to study the complete beam injection into the magnetic field, although they were also seen in calculations made by Boers (1968) for a very similar electron gun.

D. Trapping and Pulsing System

The gas to be ionized in the electron beam is injected radially into the arift tubes by a $3.2 \mathrm{~mm}$ diameter tube plugged into the wall of the first drift tube within the region where the ions are contained. This stainless steel line"is bxaught into the ionization chamber near the electron collector, and is isolated from the tube to which it delivers the neutral gas by an insulator fitting that joins it to the drift tube. The gas flow through this tube can be controlled very accurately with a Varian Model 951-5100 variable Leak Valve. This valve is bakeable and useable at pressures below $10^{-11}$ Torr, producing precisely controlled gas flow rates down to $10^{-10}$ iorr-liters/sec by the use of an optically Iiat sapphire and captured metal gasket seal.

The six drift tubes in the test EBIS have an inside diameter of $3 \mathrm{~mm}$ and the four in the containment region have lengths of $32 \mathrm{~mm}$ each, while the front tube has a length of $28 \mathrm{~mm}$ and the last tube has a length of $19 \mathrm{~mm}$. The tubes each have a gap of about $1 \mathrm{~mm}$ between them, and the first tube is mounted into the magnetic shim with an insulator so that it is approximately $1 \mathrm{~mm}$ from the electron gun anode. The potentials are applied to these drift tubes (see Fig. I(b), p. 12) by $0.5 \mathrm{~mm}$ wires threaded through the insulators used to support each tube. These wires are attached to a feedthrough in the extractor chamber, and are brought to the ionization region in an insulator that is located in a pumping slot made in the electron collector assembiy. 
The electronics system used to apply these and the other potentials in the EBIS is shown schematically in Fig. 15. The synchronizer unit simultaneously ärives the anode segulator on the electron gun and the pulse amplifier on the drift tubes so that the electron beam, as well as the ion trar, can be pulsed. A delay built into the circuits causes the electron gun to be turned on at least for $20 \mathrm{\mu sec}$ before the trapping potertial is applied to the drift tubes.

The electron gun cathode potential is variable from 0 to $-3.5 \mathrm{kV}$, and the anode can be pulsed from the cathode potential to a maximum potential of $+4 \mathrm{kV}$. This method of pulsing the electron gun gives an electron bean pulsr: with a rise time of $20 \mu \mathrm{sec}$, but it has a fall time of $2 \mathrm{msec}$, limited by the drive circuitry. The electron beam pulse width is variable fxom $30 \mu \mathrm{sec}$ to $2.2 \mathrm{sec}$ at a repetition rate of $1 / 3$ to 500 $\mathrm{Hz}$. The electron gun can also be operated in a $\mathrm{CW}$ mode by simply disconnecting the anode regulator from the synchronizer unit. The filament heating the cathode of the electron gun is driven by an ac power supply floating at the cathode potential. The power requirement for the heater is $3 \mathrm{~A}$ at $12 \mathrm{~V}$.

Trapping and extraction potentials are supplied to the drift tubes from two resistive networks driven by power supplies adjustable from 0 to $+650 \mathrm{~V}$. The trapping potential applied to each of the end drift tubes is variable independently of the plateau voltage applied to the tubes where ions are trapped, and the voltage applied to each tube can be varied by changing the connections of the tubes to the resistive network. The containment pulse width is variable from $30 \mathrm{\mu sec}$ to $1.1 \mathrm{sec}$. The rate at which the confinement potential is lowered is also variable, from 30 usec to 1 misec.

At extraction, the constant potential on the electron gun cathode is applied to the ion extractor, with an additional voltage, variable from 0 to $0.5 \mathrm{kV}$, added to this potential. This additional potential is necessary to prevent the electrons from axially escaping or striking the extractor, and it also helps to focus the ions extracted from within the electron beam in the fringing field of the magnet. The ion extractor, with an inside diameter of $6.4 \mathrm{~mm}$, can be adjusted before pumpdown with isespect to the grounded electron collector, and the whole extractor- 


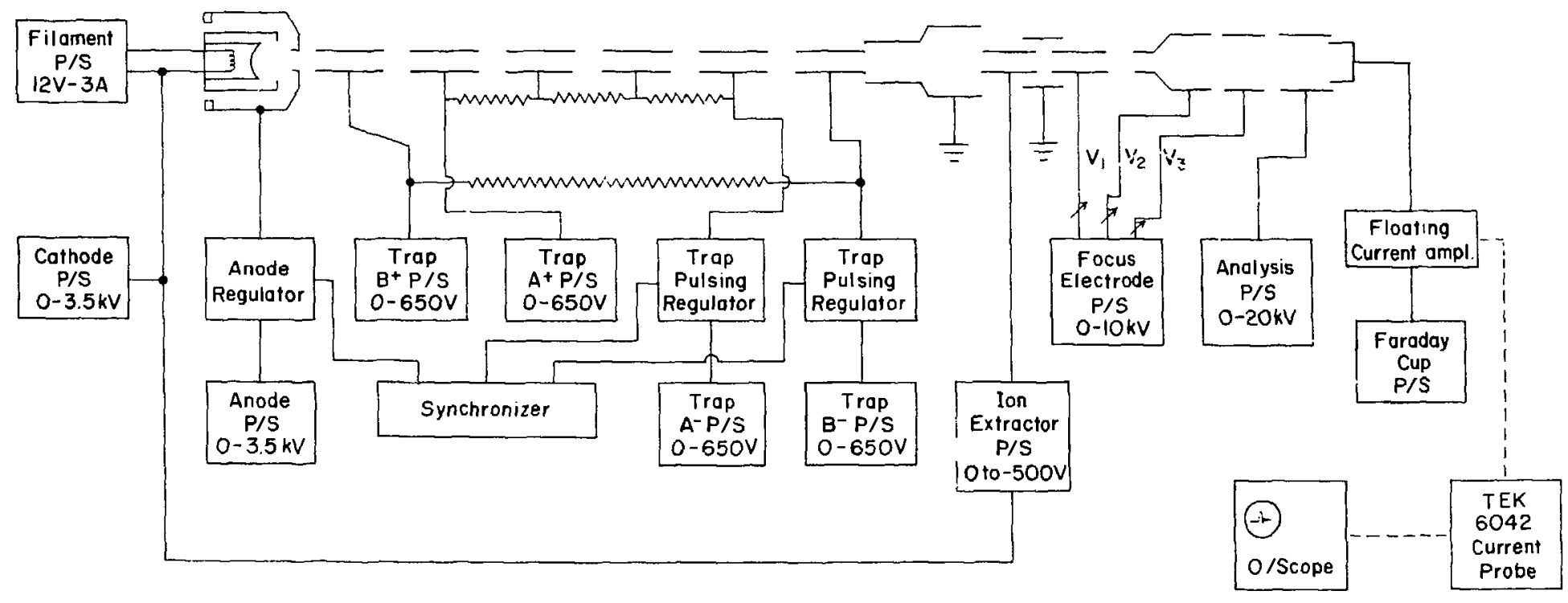

FIG. 15. Schematic of the pulsing and focussing circuits for the test EBIS. 
collector assembly is adjustable with respect to the fringing field of the solenoid during operation, as shown in Fig. 7 (p. 45).

Focussing of the extracted ion beam into the analysis system is accomplished by an asymetric electrostatic lens assembly mounted in the extractor chamber, as shown in Fig. 16. The firit element, El, is mounted just beyond the ion extractor, and the 2-3 $\mathrm{mm}$ gap between them is shielded by a groundea cylindrical electrode to preserve the cylindrical symmetry of the focussing system. This lens and ground ring are mounted on the electron collector-jon extractor assembly. In order to be able to remove the assembly from within the ionization chamber, a large gap is required between $E 1$ and the second electrostatic lens, E2. This second lens and the last lens, E3, are mounted on the extractor chamber lid. Thus, they are fixed in position with respect to each other, and with respect to the acceleration electrode attached to the analysis system. As scen in Fig. 16, the inside diameter of the lenses is progressively increased Erom the inside diameter of the extractor electrode to the $25.4 \mathrm{~mm}$ inside diameter of the acceleration tube in the analysis system. This allows for space-charge effects in the ion pulses, and also makes a long focal length possible for the last acceleration gap. The ion beam can then be focussed jnto the magnetic analysis system with the optimum condition (i.e. small magnification) for ion separation. The potentials applied to the three lenses are each variable from 0 to $-10 \mathrm{kV}$, while the potential of the analysis system, and the acceleration tube connected to it is variable from 0 to $-20 \mathrm{kV}$.

E. Analysis System

The ions extracted from the EBIS and focussed into the analysis system by the electrostatic lenses are charge state analyzed in $q / m$ by a uniform field, rectangular pole c-magnet. The analysis chamber floats at the potential of the last acceleration lens, and is isolated from the pole tip of the C-maynet by insulator sheets. The ions are deflected through an angle of $30^{\circ}$ into an isolated faraday cup floating at the voltage of the analysis system.

In order to minimize the problems of focussing with the rectangular pole magnet, the pole edge is set perpendicular to the exit direction of 

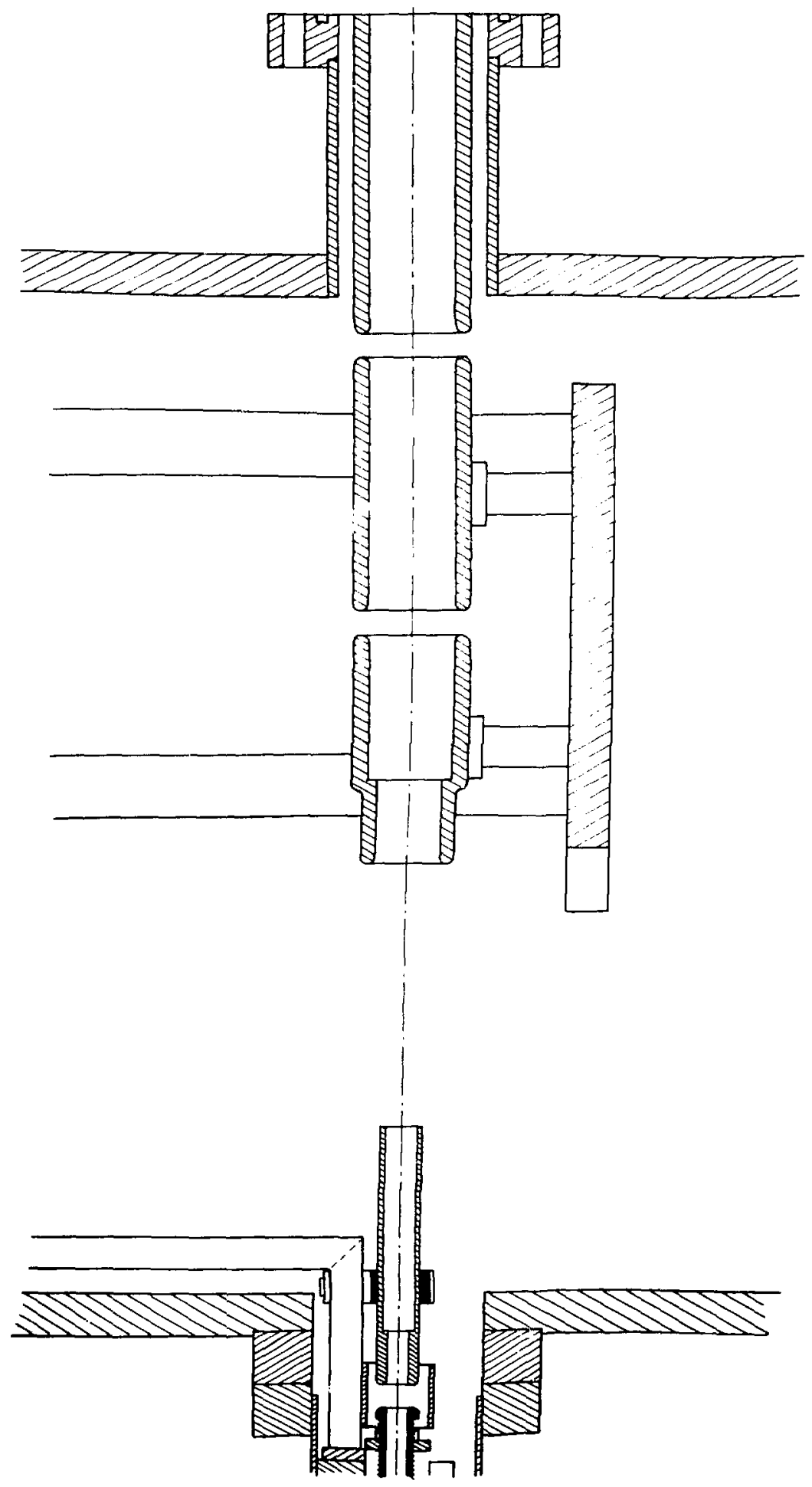

FIG. 16. Cutaway view of the ion beam focussing lenses in the EBIS. 
the deflected ion beam, and an intermediate radial and vertical image is formed at the effective entrance edge of the magnet by the electrostatic lenses. This neutralizes the astigmatic effect of the vertical magnet edge focussing, thus minimizing the effects of zero radial focussing by rectangular pole edges.

In the EBIS test configuration the electron collector is at ground potential and the ions are then accelerated into the isolated analysis system through a maximum potential drop of $-20 \mathrm{kV}$. This is the reverse of what is necessary for injection into the accelerator, but the relative focussing conditions are the same in each case, and this test configuration makes experimental adjustments on the ion source much easier than would a high voltage environment. However, the test configuration does necessitate the measuring of small ion currents at high voltages.

These measurements are accomplished in this test EBIS by an inductively coupled current amplifier. The current on the faraday cup, or on the whole analysis system, can be read as an integrated current on a meter floating at the analysis potential, or it can be switched into an isolated $x 10$ inductive loop and time monitored on an oscilloscope with the use of a Tektronix Model 26042 current meter. The current amplifier is calibrated with a Keithley Model 261 picoammeter suurce, and has full scale deflections of $100 \mathrm{nA}$ to $500 \mathrm{\mu A}$, in half decade increments. The current meter and oscilloscope can both be calibrated by manufacturers' procedures to an accuracy of $\pm 5 \%$, but the error in reading the pulse shape and height on the oscilioscope and the noise picked up on the high voltage faraday cup leads to absolute ion currents probably not accurate to better than $\pm 20 \%$. 


\section{EXPERIMENTAL MEASUREMENTS}

\section{A. Vacuum Results}

Since the vacuum requirements in the EBIS are so severe, as shown in Chapter II, much effort went into the achievement of the ultrahigh vacuum necessary for operation of this ion source. Vacuum testing was carried out on the original one meter prototype source as well as on the short test model.

The measurements on the origin. 1 ionization chamber were made with it attached to the large extractor chamber on one end, but with the electron gun chamber on the other end replaced by a blank-off tube containing a nude Bayard-Alpert ionization gauge. The opening to the ionization chamber was $3.8 \mathrm{~cm}$, and the blank-off tube was small enough to not significantly add to the gas load of the chamber. The pressure measured by this gauge was thus a good indication of the vacuum attained within the ionization region in terms of a nitrogen equivalent pressure.

After leak testing the system and attaining a pressure of less than $1.0 \times 10^{-6}$ Torr in the extractor chamber, the system wa; baked at $175^{\circ} \mathrm{C}$ for 24 hours. The titanium pump was then turned on and operated during the cooling down of the system. The liquid nitrogen dewar on the titanium pump was then filled. A pressure of $5.0 \times 10^{-9}$ Torr was measured in the extractor chamber and a pressure of $2.0 \times 10^{-8}$ Torr was measured at the end of the ionization chamber. When the cryogenic surfaces in the ionization chamber were cooled with liquid nitrogen $\left(77^{\circ} \mathrm{K}\right)$, the pressure in the extractor chamber fell to $7.0 \times 10^{-10}$ Torr and the pressure at the end of the ionization chamber fell to $1.5 \times 10^{-9}$ Torr. This pressure in the ionization chamber did not change when the valve to the analysis system was opened, and the pressure in the extractor chamber only rose to $9.0 \times 10^{-10}$ Torr. The pressure in the analysis system remained constant at $3.0 \times 10^{-8}$ Torr throughout the test period of several hours.

'The electron gun chamber was also tested separately at a later date 
with this blank-off tube set up with a faraday cup in it to make electron current measurements with the electron gun. After baking this system at $175^{\circ} \mathrm{C}$ for 24 hours, a pressure of $1.0 \times 10^{-9}$ Torr was quickly attained in the chamber, as measured by the nude Bayard-Alpert gauge located in the large flange on the rear of the chamber opposite the electron gun. This pressure only rose to $2-3.0 \times 10^{-9}$ Torr when the cathode of the electron gun was heated, but would increase beyond $1.0 \times 10^{-8}$ Torr when the electron gun was turned on for the current measurements to be described later.

Further tests on the completely assembled prototype vacuun: system were discontinued because the one meter solenoid had been found to be unuseable, and vacuum testing of the test model system began. Since the electron gun chamber and extractor chamber had already been tested separately, the entire source was assembled and tested under operating conditions.

During a typical operating cycle of this system after exposure to atmosphere, the system is first roughed down simultaneously with the mechanical pump on the turbomolecular pump and with the liquid nitrogen trapped mechanical pump on the electron gun chamber. The analysis system is roughed out separately with the by-pass valve on the pumping manifold used with the diffusion pump. When the pressure of the roughing line on the turbomolecular pump falls below 1000 microns, the turbomolecular pump is turned on to prevent backstreaming and to assist in the pumpdown. I,y the time the turbomolecular pump has reached the maximum speed of the rotors, the pressure in the extractor chamber is below $1.0 \times 10^{-5}$ Torr. The mechanical pump on the electron gun chamber is valved off and a varian helium leak detector is used as the backing pump for the turbomolecular pump in order to helium leak test the system at a pressure on IG-02 below $1.0 \times 10^{-6}$ Torr. After the system has been leak tested, the liquid nitrogen trapped mechanical pump on the electron gun chamber is opened up again and the Electro-Ion pump is turned on. The mechanical pump is left on during the automatic bake cycle of the pump and afterwards until the pressure in the pump has reached about $5.0 \times 10^{-5}$ Torr. The mechanical pump is then valved off and the Electro-Ion pump and turbomolecular pump are allowed to pump 
several hours. The pressure in both chambers is now usually below $1.0 \times 10^{-7}$ Torr. The entire system, except for the Electro-Ion pump, is then baked at $175^{\circ} \mathrm{C}$ for 24 hours, the pressure in the chambers usually rising to above $1.0 \times 10^{-5}$ Torr during the first few hours and falling slowly after that to an equilibrium pressure of about $1.0 \times 10^{-7}$ Torr. Before the system is cooled, the titanium filaments in the titanium pump and the two nude ion gauges in the system are degassed. During the cool down time the titanium sublimation pump is operated about once every $1 / 2$ hour without liguid nitrogen in the dewar. After the entixe system has cooled off, the pressure in the electron gun chamber is $5.0 \times 10^{-9}$ Torr and the pressure in the extractor chamber is $21.0 \times 10^{-8}$. Torr. The electron gun cathode heater is then brought on and degassed at a power of about 20 watts (heater voltage of $9 \mathrm{v}$ ), and the cryogenic surfaces in the titanium pump and ionization chamber are cooled to $77^{\circ} \mathrm{K}$. The final pressure in the electron gun chamber is then about $1.5 \times 10^{-9}$ Torr with the electron gun off and about $3.0 \times 10^{-9}$ Torr with the electron gun in operation. These pressures are about one-half of these values after several days of ion source operation. The final pressure attained in the extractor chamber is usually about $2.0 \times 10^{-9}$ Torr, and it also decreases slowly as the source is pumped for several days. In fact, pressures of $7.0 \times 10^{-10}$ Torx have been obtained in both chambers on several early experimental test runs.

The analysis chamber is evacuated by the diffusion pump system with the usual procedure for such a system, and the usual operating pressure of $3-5.0 \times 10^{-8}$ Torr is achieved in a few hours without baking unless the chamber has been exposed to the atmosphere for a prolonged period of time. As in the vacuum tests with the prototype, the analysis pressure remains constant when the valve to the extractor chamber is opened, and the pressure in the extractor chamber only increases about $20 \%$ in the test model system.

During one of the operating runs, residual gas spectra were taken with a Grariville Phillips Spectra Scan 750 residual gas analyzer attached to the extractor chamber. These spectra were taken at several starjes of the pumpdown process and are shown in Fig. 17. The first syectrum was taken after the system had been evacuated with only the 


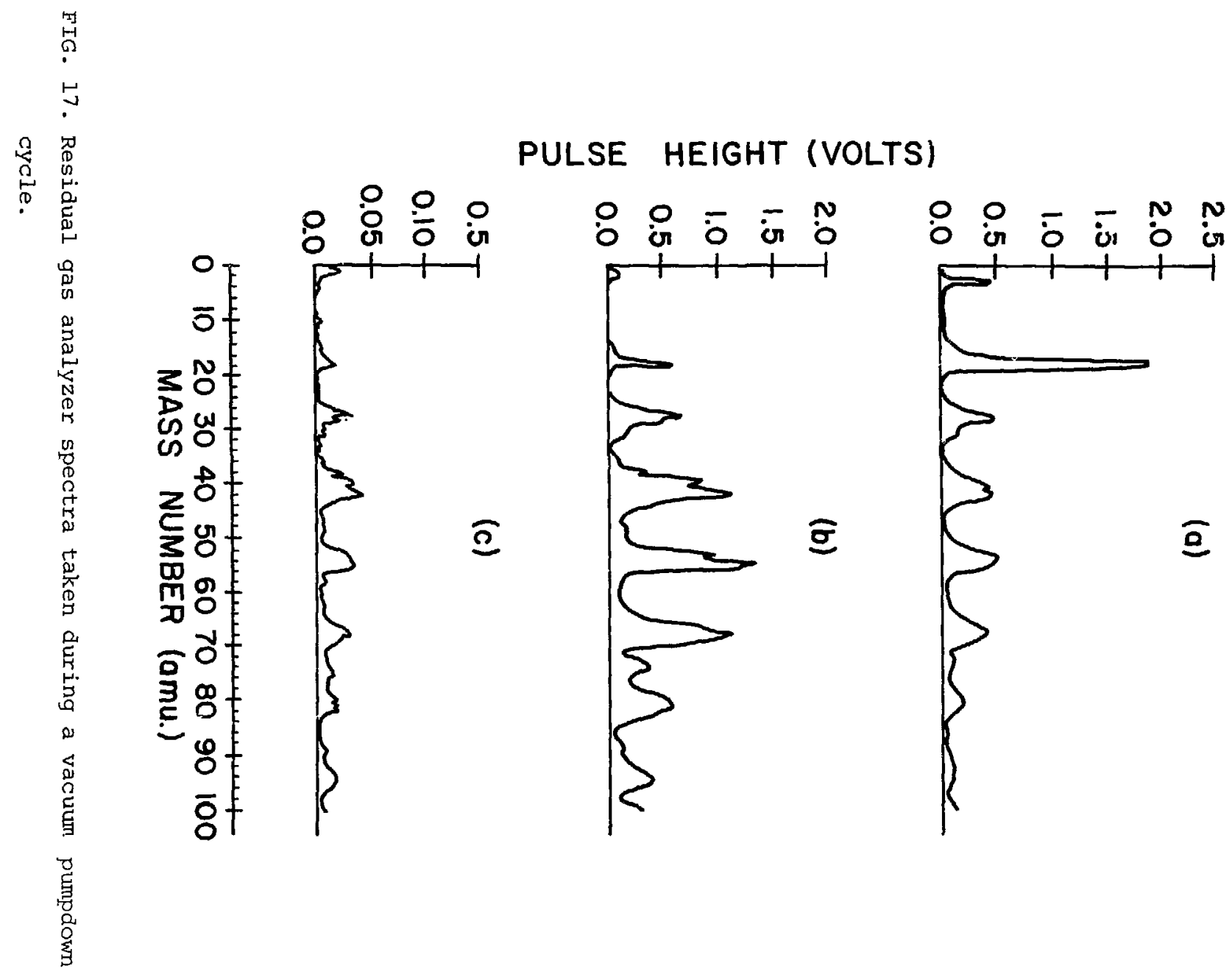


turbomolecular pump to a pressure of $1.7 \times 10^{-7}$ Torr. in the extractor chamber. The largest component in the residual vacuum is clearly the water vapor, with peaks of hydrogen, nitrogen and rarbon monoxide, as well as heavier gases and vapors also present. In the second spectrum, the titanium pump had been turned on before the system was baked, with the pressure in the extractor chamber dropping to $4.3 \times 10^{-8}$ Torr. This spectrum clearly shows the effectiveness of the titanium in removing the hydrogen and water varor in the system.

The last spectrum was taken after the system had been baked for 24 rours at $175^{\circ} \mathrm{C}$ and the cryosurfaces had been cooled to $77^{\circ} \mathrm{K}$. The ultimate pressure, after the titanium pump had been used for several hours, reached $2.0 \times 10^{-9}$ Torr and the residual gas is seen to consist of very small quantities of all of the gases present before the bake-out, but with the lighter gases reduced by the largest factor. All of these peaks are consistent with the gases given off by the polymers used in the ion source, with a few also from the other materials. The lack of any oxygen peak indicates that the system is indeed leak tight, but the peak at mass 57 indicates that there has, however, been some backstreaming of mechanical pump oil through the cold trap or turbomolecular pump. Some of the peaks in the final spectrum may also be due to contamination of the vacuum system by the residual gas analyzer itself since it gave rise to a pressure increase when first turned on even though it had been baked. In the experimental runs after the residual gas analyzer was removed, the ultimate pressure attained was lower than when the analyzer had been attached to the system.

B. Electron Beam Measurements

As mentioned previously, tests have been made on the electron gun to check the electron current given off by the cathode as well as to study the focussing of the electron beam through the magnetic shim and solenoid. The electron beam current is shown in Fig. 18 as a function of the operating voltage of the electron gun. The points measured by the manufacturer, Hughes Aircraft company, are included in the data, and the theoretical current for a constant perveance of $2.0 \times 10^{-6} \mathrm{~A} / \mathrm{V}^{3 / 2}$ is also shown. The data shown in this figure include current measurements 


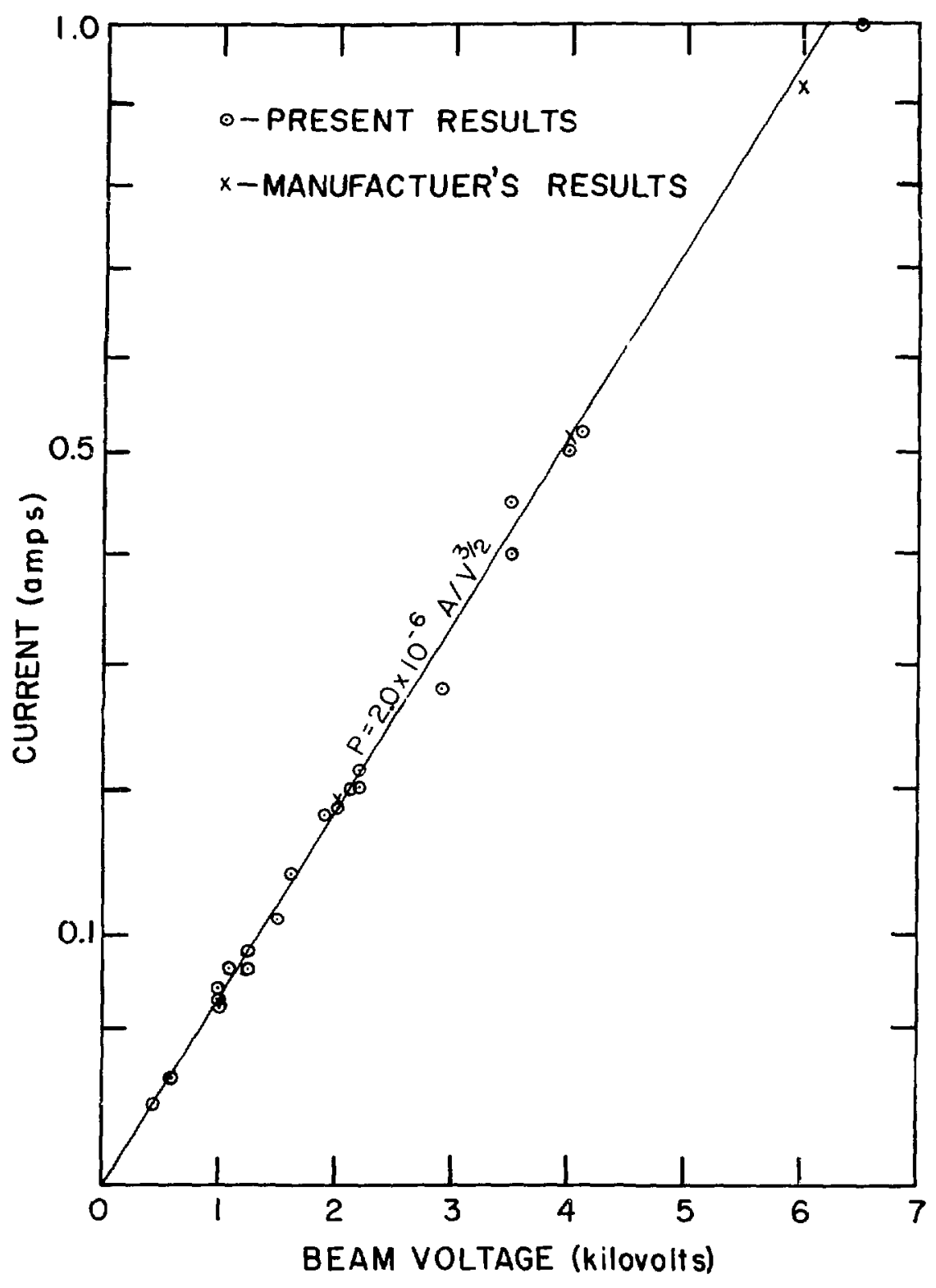

FIG. 18. Electron beam current as a function of the operating voltage plotted on a $2 / 3$ power graph for the $112-2 B$ electron gun. 
on the electron gun with the blank-off mentioned earlier, as well as the measurements made during injection of the electron beam into the magnetic field. In all cases the cathode heater power was approximately 18 watts, giving a cathode temperature of $\sim 1150^{\circ} \mathrm{C}$.

Measurements were made on the injection of the electron beam into the original one meter solenoid with a pinhole aperture that could be moved across the electron beam. The aperture had a diameter of $0.25 \mathrm{~mm}$ and the current that passed through it was collected on an isolated faraday cup. The measurements made with this device showed that the electron beam had a very rippled structure, and that it was located $3 \mathrm{~mm}$ off of the axis of the solenoid after travelling only $30 \mathrm{~cm}$ into it. Further magnetic field measurements revealed that the magnetic shim was not sufficient to prevent cathode flux linkage, and that the magnetic axis of the solenoid had the laxge local variations described earlier. These injection tests were discontinued and the magnetic shim and solenoid were removed and reconstructed.

The new magnetic shim and solenoid were used in the test model EBIS described in the previous chapter, and electron beam injection and transmission has been studied with this system. The transmission of the electron beam through the six drift tubes in the test model is shown in Fig. 19 for two different operating voltages. The theoretical value of the Brillouin focussing field is indicated for each curve. The focussed electron beam radius used for calculating the Brillouin field was taken to: be 80 \% of the unfocussed electron beam minimum, as shown earlier to be the case for this particular set of entrance conditions. It can be seen that the transmission of the electron beam through the $3 \mathrm{~mm}$ i.d. drift tubes exceeds $99.5 \%$ for a focussing field about 16\% greater than the theoretical value.

The electron beam transmission was achieved by minimizing the current measured on the first 5 drift tubes by adjustment of the position of the solenoid with respect to the beam axis with a focussing field less than the Brillouin value. Then, with the focussing field turned up above the Brillouin value, there is virtually no loss on these tubes. The current lost on the last drift tube can then be minimized by external shimming of the magnetic field in the extractor region to give the 


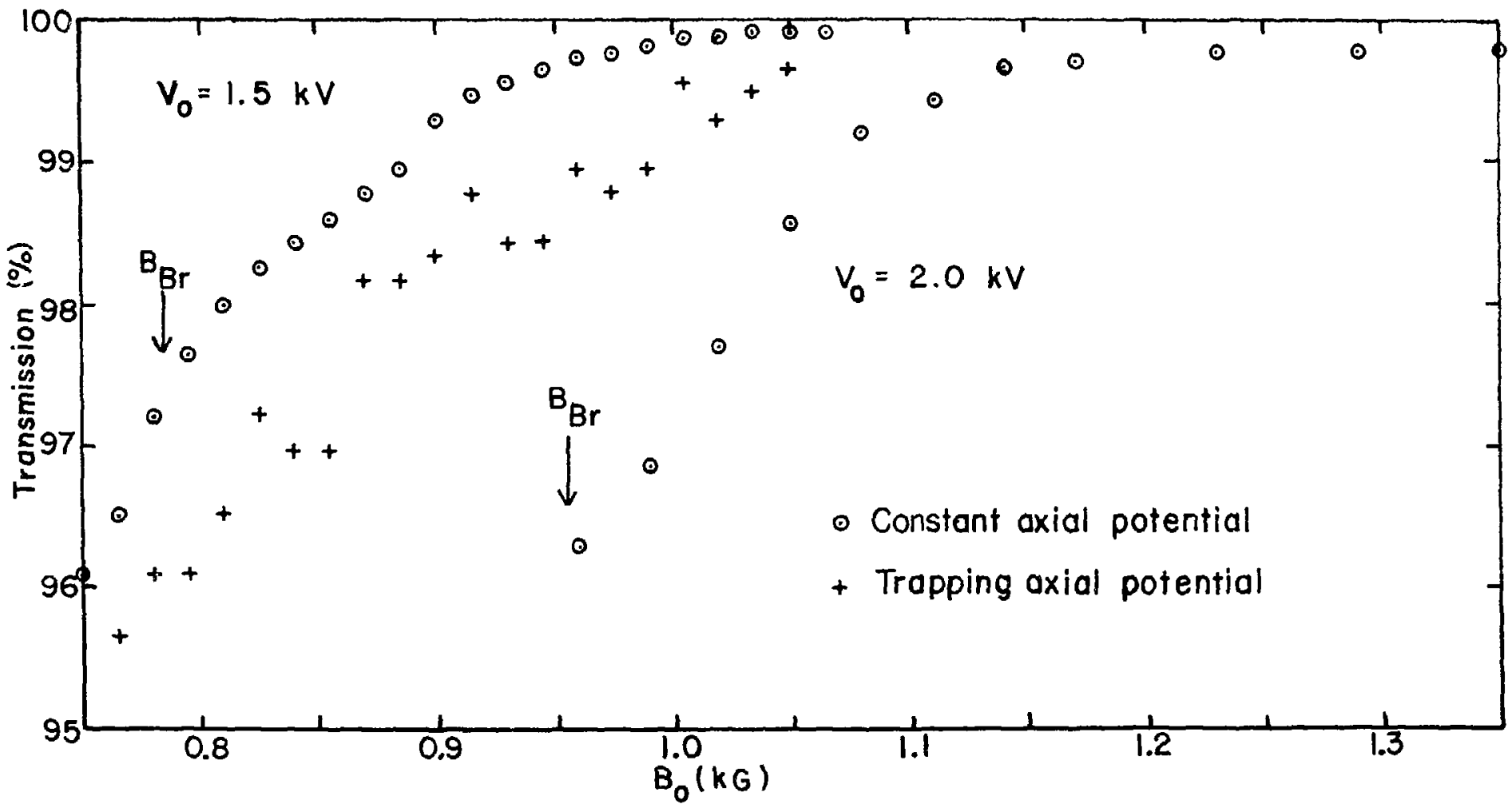

FIG. 19. Electron beam transmission through the test model EBIS for two different electron gun potentials with the theoretical Brillouin focussing field value indicated for each. 
transmission measured above. The current lost on this last drift tube is still, however, tire largest part of the total beam lost, indicating that the magnetic axis has a sharp curvature in the fringing field on this end.

The transmission of the electron beam was measured first with all the drift tubes at the constant potential of the electron gun anode. Under ideal conditions, this condition would maintain perfect Brillouin flow for a beam entering under the proper entrance conditions. The trapping potentials recessary for operation of the EBIS were then applied and the transmission of the electron beam decreased as shown also in Fig. 19 (p. 74). As before the trapping rotentials were applied, most of the current loss was on the last drift tube, with only a very small increase in the losses on the other five drift tubes. The large fluctuations in the transmission as well as the decrease in transmission indicate that the electron beam focussing is disturbed by the trapping potential and the electron beam becomes scalloped. This effect was qualitatively seen to decrease in magnitude as the electron energy was increased. Since the trapping potentials remained essentially constant, this defocussing effect is then due to the potential differences that are necessary to trap the ions axially with the electron beam.

C. Ion Production Results

After an operational vacuum had been attained and the transmission of the electron beam through the ion source had been optimized, measurements of the total ion current produced in the ion source during a containment cycle began. When the positive ion output had been optimized, the ion beam was focussed into the analysis system. The transmission of the ion beam into the analysis system was studied by measuring the ion current soing straight through the system into the faraday cup placed at the end of the beam line. The ion beam was then analyzed by the use of the magnetic field and the faraday cup placed at the end of the $30^{\circ}$ bend.

The ion measurements were carried out at lower electron energies than the projected operational value of $6 \mathrm{kV}$ in order to reduce the arcing in the source and to keep the outgassing due to electron bombard- 
ment to a minimum. The total ion sutput measured in the analysis system during a containment cycle is shown in Fig. 20. The small level of ion current that is aetected at all times during the electron gun pulse is due to ionization that occurs in the region between the ion extractor and the end of the containment trap. The main ion pulse is seen to appear at the end of the containment time, and integration of the charge in this pulse reveals that the space-charge compensation in the electron beam is almost complete. Table 3 gives the operating parameters and actual space-charge compensation obtained during source operation with residual gas.

TABLE 3. Space-charge compensation observed in the Texas A\&M EBIS.

\begin{tabular}{ccccc}
$E_{e}(\mathrm{keV})$ & $\mathrm{I}_{\mathrm{e}}(\mathrm{A})$ & $\mathrm{N}_{\mathrm{e}}\left(\mathrm{e}^{-}\right)$ & $\mathrm{iT}^{+}\left(-\mathrm{e}^{-}\right)$ & $\mathrm{N}^{+} / \mathrm{N}_{\mathrm{e}}$ \\
\hline 2.25 & 0.235 & $6.8 \times 10^{9}$ & $4.5 \times 10^{9}$ & 0.66 \\
1.90 & 0.175 & $5.5 \times 10^{9}$ & $5.0 \times 10^{9}$ & 0.91 \\
\hline
\end{tabular}

After the extracted ion pulses had been optimized to maximum spacecharge compensation, the total ion current was measured as a function of the containment time. Such a measurement is shown in Fig. 21 for the last set of ion source operational parameters given in Table 3 above. The space-charge neutralization of the electron beam in only a few milliseconds is a clear indication that the vacuum in the ionization region is not sufficient for production of highly-charged heavy ions. This was true for all of the experimental measurements, since the space-charge neutralization time was always less than 3 milliseconds for all of the experimental runs. However, the observed ionization of residual gas during this time was sufficient to study ion production within the EBIs with the external electron yun, and to determine the conditions that would be necessary fuc the EBIS to produce ions for injection into the cyclotron.

T.fter the ion source became operational and the output of positive ions had been detected, it was found that the ions could not be transported through the analysis system. An ion collector was placed in the 


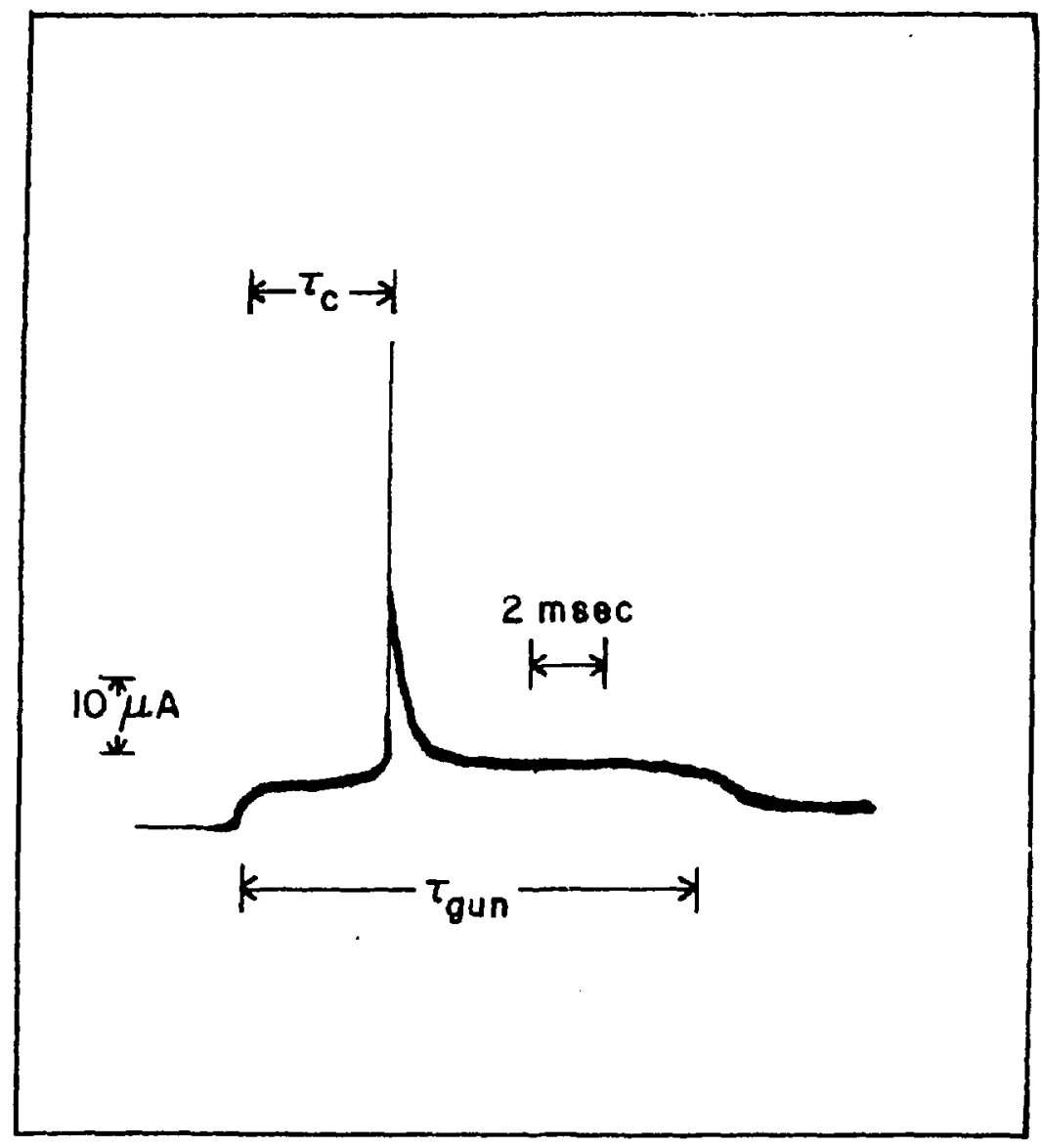

FIG. 20. Ion current during a typical operating cycle of the EBIS. The electron beam pulse is indicated by $\tau_{\text {gun }}$ and the containment time for the ions by $\tau_{c}$. 


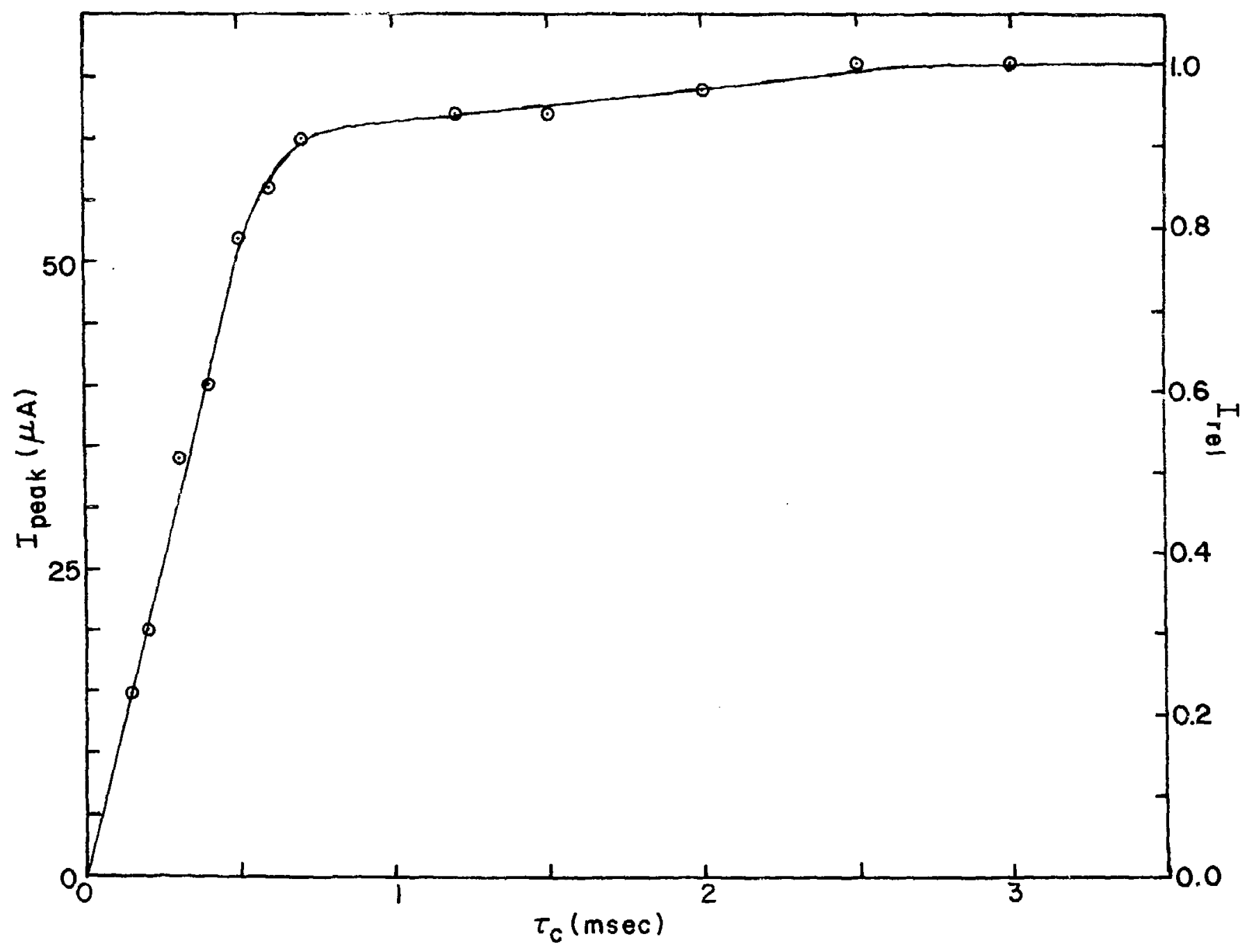

FIG. 21. Total ion current from the test EBIS as a function of the ion containment time. 
beam line after the gate valve $\mathrm{V}-03$ and after several realignments of the electron gun, drift tubes, ion extractor and focussing lenses, most of the ions could be focussed through a $1.59 \mathrm{~cm}$ hole onto the ior collector.

Using this ion collector the energy spread of the ion beam was qualitatively found to be approximately equal to the potential difference along the drift tubes during their extraction. This measurement was done by extracting the ions and focussing them into the beam line which was at ground potential, and applying a positive retarding potential to the ion collector. The ion current on the collector decreas ed with increasing voltage until the voltage was the same as the potential drop along the drift tubes during extraction, at which point the current on the collector went to zero.

The ion collector was then removed and the ion beam was transpurted through tise analysis system. The ion beam was detected on the faraday cup placed at the end of the beam line going straight through the analyzing magnet, and the transmission was optimized by moving the magnet and analysis cha:ber. The optimum position of the analysis system was found to be very far from the optical alignment, indicating that the ion beam was still being stoered very badly during extraction, and possibly through the initial focussing lens. The ion beam transmission through the $1.59 \mathrm{~cm}$ hole onto the ion collector placed in the beam line was $280 \%$, but the transmission through the $2.54 \mathrm{~cm}$ apertures at both ends of the analysis chamber and into the beam line after the analysis chamber was only $40 \%$. The transmitted ion beam and the total extracted ion beam both had the same time structure, and since the focussing was by electrostatic lenses, it was assumed that the relative ionic comp:sition of both beams was the same.

This transmitted ion beam was then analyzed magnetically by bending the ions through a $30^{\circ}$ bend into a faraday cup located behind a $4 \mathrm{~mm}$ slit. A charge state sfectrum for the residual gas is shown in Fig. 22. The final energy of the ions was $10 \mathrm{~g} \mathrm{keV}$, where $\mathrm{q}$ is the charge state of the ion, and the containment time for these ions was 7 milliseconds. The dip below zero current on both sides of the $\mathrm{H}^{+}$ 


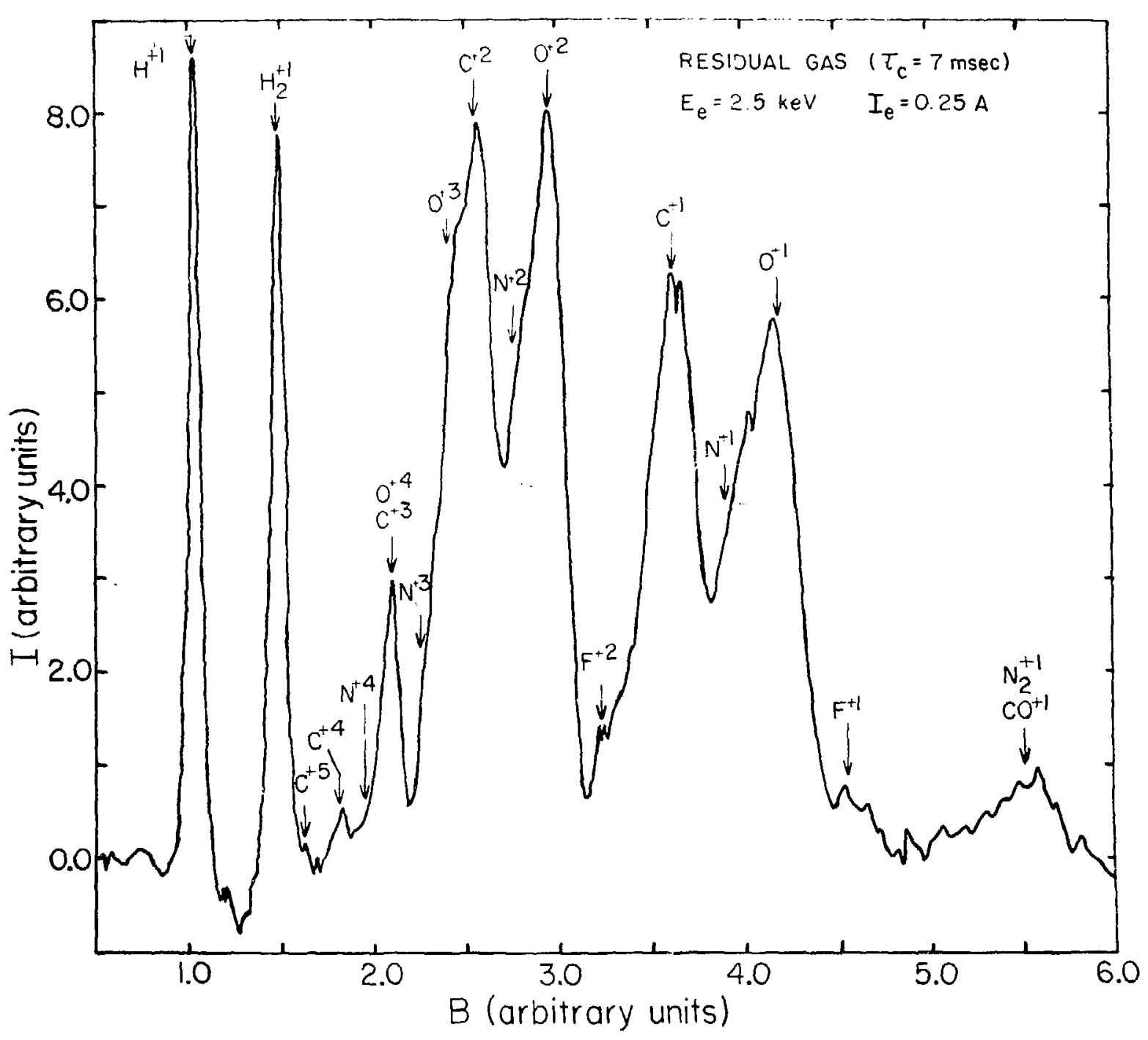

FIG. 22. Charge state spectrum for the residual gas with the indicated operating conditions. 
peak is due to secondary electrons from the slit in front of the faraday cup. The position given for various peaks has been calculated from the relative position of the $\mathrm{H}^{+}$and $\mathrm{H}_{2}{ }^{+}$peaks, and only relative current has been measured, although the measured total ion current indicated that space-charge neutxalization of the electron beam in this case was almost complete.

Although the space-charge neutralization of the electxon beam in only a few milliseconds prevented the attainment of very high charge states, the ionization of argon gas was attempted by continuous injection of the gas into the drift tubes as described earlier. The flow of argon gas was very critical, and the charge state spectrum in Fig. 23 was Laken with the optimum gas flow of $4.0 \times 10^{-9}$ Torr-liters/sec. As * before, the peak locations are calculated from the relative position of the $\mathrm{H}^{+}$and $\mathrm{H}_{2}^{+}$peaks and only relative ion currents are indicated. The highest charge state of argon that is clearly distinguishable in this spectrum is the +6 , although there appears to be a contribution due to +8 ions. As in the residual gas data, the final ion energy for the ions was $10 \mathrm{q} \mathrm{keV}$, where $\mathrm{q}$ is the charge state, but increasing this energy to $15 \mathrm{keV}$ still did not resolve the ion peaks any better in either spectrum.

Before the ion source measurements were completed, the $4 \mathrm{~mm}$ slit in front of the faraday cup in the analysis system was replaced with a $2 \mathrm{~mm}$ slit and the residual gas spectrux shown in Fig. 24 was taken. The ion source had not been baked after being opened to the atmospiere before this run and the residual pressure was several times higher than the pressure in the ion source when the previous resilual gas spectrum in Fig. 22 ( $\mathrm{p} .82$ ) was made. This is clearly reflected in the ratio of $\mathrm{H}^{+}$to $\mathrm{H}_{2}{ }^{+}$ions in the two spectra and in the relative magnitude of the $\mathrm{CO}^{+}-\mathrm{N}_{2}^{+}$peak. However, the increase in the resolution of the analysis system is apparent. This spectrum clearly shows that there were no actual air leaks into the ion source, a conclusion that was also given by the residual gas analyzer data discussed earlier. This is apparent in the spectrum from the small number of $\mathrm{O}_{2}{ }^{+}$ions seen, and also from the relatively small number of nitrogen ions seen in all charge states. 


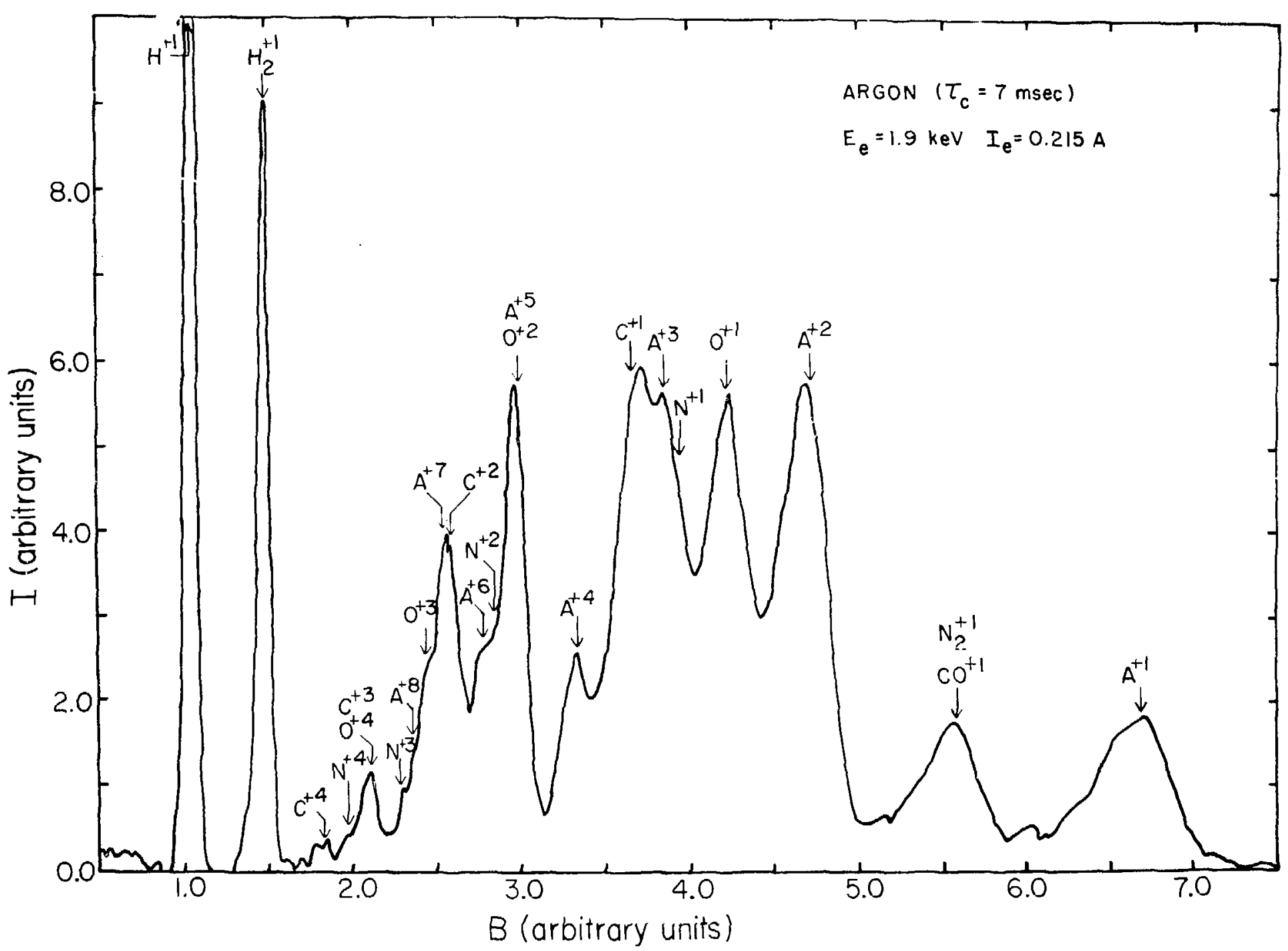

FIG. 23. Charge state spectrum for axgon gas with the indicated operating conditions. 


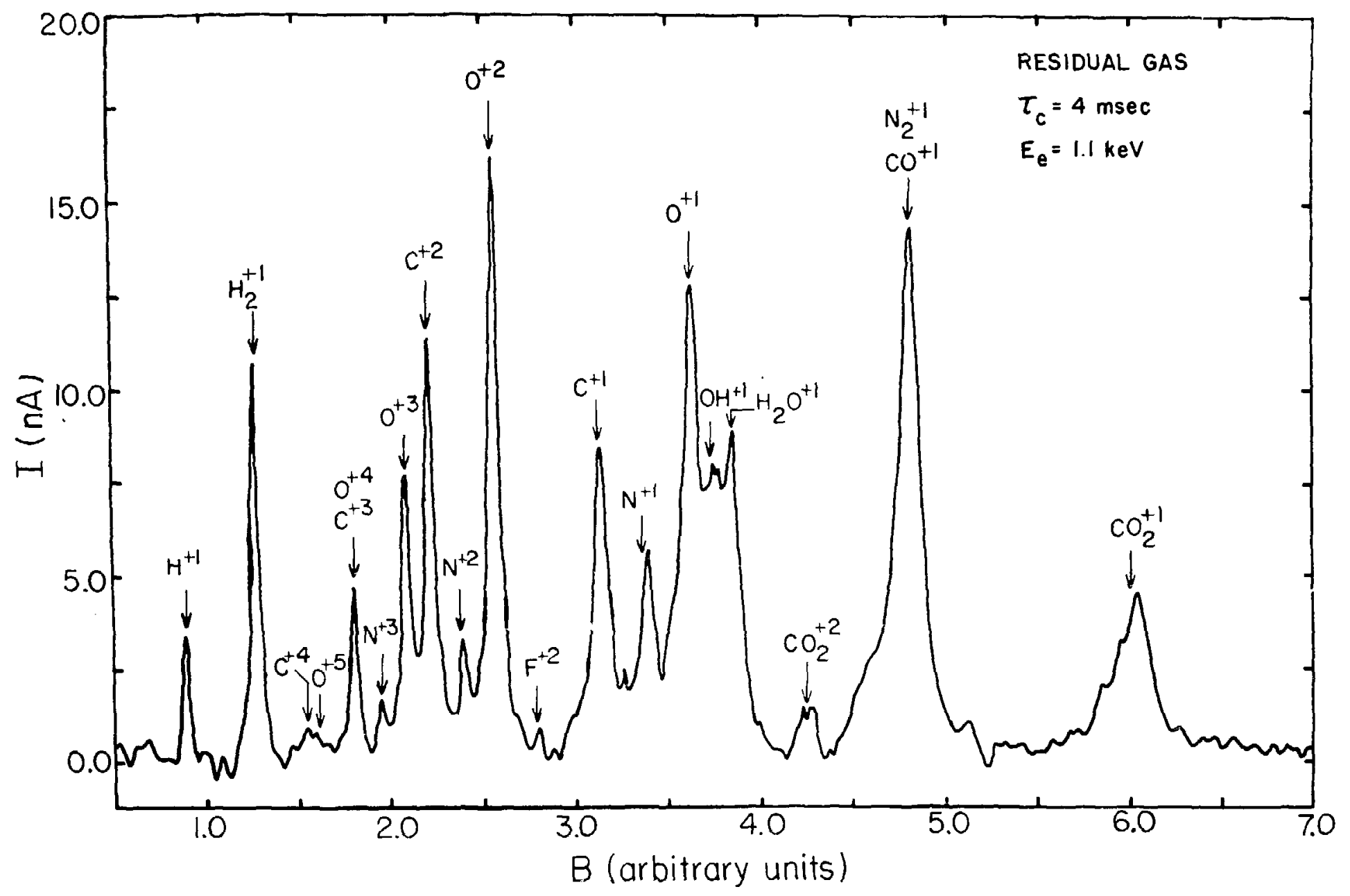

FIG. 24. Charge state spectrum for the residual gas taken with a $2 \mathrm{~mm}$ slit before the faraday cup. 
In this spectrum, the intensity of ions in the $0^{+2}$ peak was measured. The ion peak measured in the faraday cup after the $2 \mathrm{~mm}$ slit is shown in Fig. 25. The current in this peak is about $0.75 \mathrm{nA}$ with the ion source operating at 10 pulses/sec, allowing the absolute scale to be placed on the spectrum in Fig. 23 (p. 86). This peak remained the same size and shape, however, even when the ion source was operated at $100 \mathrm{pulses} / \mathrm{sec}$. The total integrated current in the spectrum is only $40 \%$ of the total current measured from the ion source, so the absolute scale takes this transmission efficiency into account.

This spectrum also indicates the means by which the time evolution of ions produced in the ion source could be extracted from the data taken on the residual gas using the larger slit. Since the peaks in the charce state spectrum all have the same shape, that of a gaussian distribution, and the width varies little for all the peaks above the $c^{+l}$ peak, the relative value of the peak heights can be used to study the ratio of ions of a particular atom as a function of containment time. In the low resolution data the charge states of carbon are the easiest to distinguish, so the time evolution of carbon ions was extracted from all the data taken with an electron energy of $1.9 \mathrm{keV}$.

These data were taken during several different experimental runs and only the data which had an $\mathrm{H}^{+}$ion peak larger than the $\mathrm{H}_{2}{ }^{+}$ion peak were used in order to eliminate data taken under bad vacuum conditions. Also, only the data taken by first maximizing the ion current through the analysis system were used. In taking the ratio of the peak heights for the carbon charge states, the slight broadening of the lower charge state peaks was ignored because of the continuous background of these ions created in the extractor region. Alsu, to extract the peak height of $\mathrm{C}^{+3}$ ions from the peak containing $\mathrm{O}^{+4}$ and $\mathrm{C}^{+3}$ ious, the decrease of ions from $\mathrm{O}^{+2}$ to $\mathrm{O}^{+3}$ was linearly extrapolated to yield the $\mathrm{O}^{+4}$ contribution. A final percentage of 75 \% was used for the $\mathrm{C}^{+3}$ contribution, although a percentage of as low as 608 didn't change the experimental ratios significantly.

These extracted ratios of carbon ion currents are shown in Fig. 26 as a function of the containment time of the ions. The salculated ratios fit by varying the unknown operating parameters of the ion source 


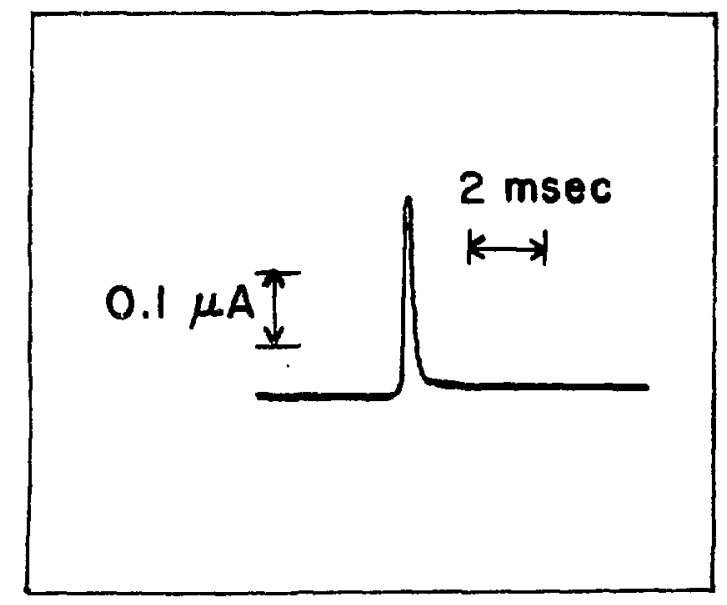

FIG. 25. Measured peak of ions passing through a $2 \mathrm{~mm}$ slit into the analysis system at the maximum of the $0^{+2}$ ion peak.

during these measurements are also shown. The calculation of these ratios takes into account the residual gas pressure and the continuous outgassing of atoms in the ionization region, but it does not consider the process of space-charge compensation in the electron beam. The effect this process has on the ion evolution is clearly revealed by the space-charge compensation data shown also in Fig. 26.

Because of the approximations made in determining the ion current ratios, the theoretical fit was made using the semi-empirical Lötz cross sections, and it can only be considered as a qualitative description of the ion evolution. However, these calculated ratios appear to give the proper description of the ion evolution when the space-charge compensation data is also taken into account. The theoretical fit also produced the reasonable operating parameters of $10^{-8}$ Torr for the residual gas pressure, $10^{-\theta}$ Torr-liters/sec- $\mathrm{cm}^{2}$ for the outgassing rate, and $16.3 \mathrm{~A} / \mathrm{cm}^{2}$ for the average current density. The ion evolution appears, using this fit, to be proceeding as a simple successive ionization frocess until the space-charge compensation of the electron beam becomes appreciable. Then, the experimental results indicate that the ions remain trapped and the charge state distribution changes very slowly. 


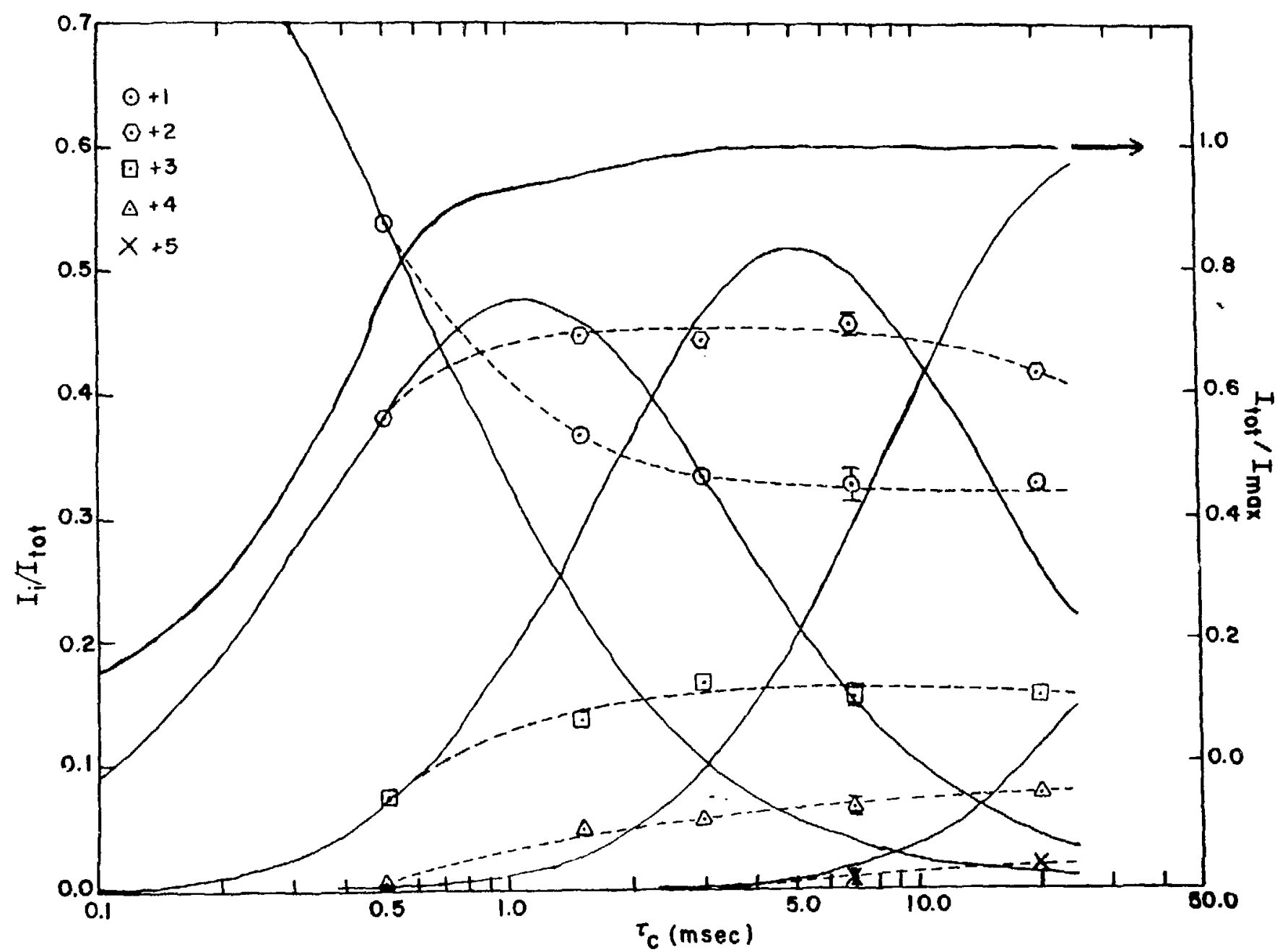

FIG. 26. Time evolution of carbon ions at an electron energy of $1.9 \mathrm{keV}$ and electron current of $0.205 \mathrm{~A}$. The theoretical fit using continuous gas injection is shown, as well as the space-charge compensation of the electron beam. 
The higher charge states appear near the predicted thresholds for observation "but the fraction of higher charge states remains very low, appearing to increase very slowly with containment time. This interpretation is indicated in Fig. 26 (p. 91) by the dashed lines drawn through the experimental data. Also indicated in this figure are the errors extracted from several residual gas spectra taken at this energy for a containment time of 7 milliseconds. These errors include the reproducibility of the data and the error in extracting the data from the charge state distribution plots. These errors were due to noise and the drift of the amplifiers in the detection system.

This continued evolution of ions within the ion source can also be seen in the argon data. As discussed earlier, a contribution of $A^{+8}$ ions appears in the argon data at a containment of 7 milliseconds. The space-charge neutralization of the electron beam was less than 2.5 milliseconds for this data, and the maximum current occurs in charge states +2 and +3 . By scaling the current density used in the residual gas data, the normalized containment time for space-charge neutralization is calculated to be less than $0.043 \mathrm{sec}$ and the normalized containment time for this observation of $\mathrm{A}^{+8}$ ions is found to be $0.119 \mathrm{sec}$. In the calculations for argon ion production at this energy, the threshold for observation of $A^{+8}$ ions is found to be at the normalized containment time of $0.10 \mathrm{sec}$, and the maximum of $\mathrm{A}^{+3}$ ions occurs at the normalized containment time of $0.04 \mathrm{sec}$, values that agree very well with the experimental observations.

The current density determined by the theoretical fit to the residual gas data can also be used to determine the average focussed electron beam diameter. Assuming a uniform density electron beam, this current density of $16.3 \mathrm{~A} / \mathrm{cm}^{2}$ yields a beam diameter of $1.26 \mathrm{~mm}$. This value is approximately $90 \%$ of the unfocussed beam minimum diameter at this energy. This is in agreement with the calculated bean diameter of $1.12 \mathrm{~mm}$, which is $80 \%$ of the unfocussed beam minimum diameter. The small discrepancy is due to the thermal effects that are not included in the calcula-

${ }^{4}$ This threshold for observation is simply the point at which the population becomes a significant fraction of the total current, approximately $0.1 \%$, and then can be observed experimentally. 
ted diameter of the focussed beam but are included in the experimentally determined beam diameter.

Finally, because of the apparent problem of ion beam steering in the extractor region very little can be said about the emittance of the extracted ion beam. The only olservation made of the ion beam's size was that the main part of the ion beam could be transmitted through the $1.59 \mathrm{~cm}$ hole located approximately one meter from the extractor with only a very small transverse motion of the ion extractor and first einzel lens assembly. During this observation the ion beam appeared to be made up of a small inner beam of high density with a larger more diffuse beam around it. When only the diffuse: beam was transported to the analysis system only very low residual gas charge states could be seen. indicating these ions were possibly produced only in the extractor region during the electron beam stopping process.

All the data taken on the test model EBIS thus gives a consistent interpretation of the processes taking place within the ion source, and these processes are described qualitatively, if not quantitatively, by the observed parameters of the ion source. The ultimate residual vacuum attainable in the ionization region appears to be the primary limitation in the test model EBIS, causing the electyon beam to be space-charge neutralized before the highly-charged ions can be produced within it. The electron beam current and energy are also limited in the present ici. source, not by the entrance conditions of the electron beam from the external gun into the solenoid, but by the problem of a non-linear magnetic axis in the focussing field of the solenoid. Also, even though the electron beam appears to be space-charge neutralized by the ions within it, the study of these ions is made very difficult due to their steering in the extractor region by the curvature in the magnetic axis of the solenoid or by a slight misalignment of the extractor electrode and lenses in the fringing field of the solenoid. The ions extracted from the ion source have an energy spread at least as large as the potential gradient used in the ionization region during extraction, and the ions are also trapped even after the space-charge neutralization of the electron beam has been achieved. The extraction of ions from within the ionization volume is essentially complete, indicating that the EBIS can be 
useful as a source of multi-charged heavy ions if the proper operating conditions are achieved. This indication is also supported by the reproducibility of the operating conditions in the ion source, the low gas consumption needed to operate the source, and the long lifetime of the source, with it being operated for more than a week on several experimental runs. 
CHAPTER V

DISCUSSION OF RESULTS AND CONCLUSIONS

The resưlts obtained with the test model EBIS on the production of multi-charged ions of the residual gas and argon have shown that such an ion source has the potential of being useful for atomic, and even nuclear physics studies. These results have shown that the use of an external convergent electron gun does not make the operation of such an ion source more difficult while it does make the attainable current density much higher than with other types of electron beam focussing. Although the residual vacuum limited the containment time and the magnetic field inhomogeneity limited the maximum current and energy of the electron beam, the ions obtained with the test model EBIS clearly indicate that the operation of this source is understood. These results have shown that the ionization process is predominantly successive electron impact ionization, since the ions increase in a calculable manner under this process until the electron beam has become space-charge compensated by the ions trapped within it.

The space-charge neutralization of the electrc, $n$ beam has been experimentally measured to be greater than 90\% in the test model EBIS, in spite of the steering problems that occurred in the extraction region, indicating that the condition of quasi-neutrality is experimentally achieved. Also, the small potential trap first postulated by Linder and Hernquist (1950) does exist since the ions continue to be trapped within the electron beam after space-charge compensation of the beam is complete. Experimentally the ion charge state distribution continues to evolve, but very slowly. The detection of higher charge states even after the electron beam has been space-charge compensated is an indication of this continued evolution. The very slow increase in the number of high charge states indicates that they escape from the potential trap almost as fast as they are created. However, since the total charge appears almost constant, the appearance of these ions, as well as the decrease in the number of lower charged ions indicates that the ion tem- 
perature of many of these highly-charged ions is less than that of the remaining small potential depression in the electron beam. This indicates that the ion temperature proposed by Wieseman (1972) is useful in describing the ions extracted from the EBIS, and the qualitative observations made on the test model do indicate that the extracted beam has an emittance that is small enough to make it suitable for injection into an accelerator.

A thorough study of the ions within the EBIS and the ionization and loss processes was not possible with the test model because of the technical problems present in the operation of this particular ion source. The operation of the test model did however show that if these few technical difficulties are overcome, the EBIS will perform as predicted in the theoretical calculations, provided the correct cross sections for ionization are used. The most important of the technical difficulties to overzome are the achievement of a good vacuum and the construction of a focussing solenoid with a very linear magnetic axis and complete cylindrical symuetry of the magnetic field.

The measurements made on the vacuum system of the test model EBIS indicated that the system was void of any air leaks but was still not able to be pumped down to the design pressure. The calculated pressure in the ionization chamber of the test model was $5.0 \times 10^{-10}$ Torr and the calculated pressure in the extractor chamber was $1.3 \times 10^{-10}$ Torr. The working pressure measured in the extractor chamber during the experimental measurements was approximately $2.0 \times 10^{-9}$ Torr. By using the raisio of the theoretical pressure in the two regions, the working pressure in the ionization chamber is calculated to be approximately $8.0 \times 10^{-9}$ Torr. This pressure agrees with the pressure of $1.0 \times 10^{-8}$ Torr obtained by fitting the carbon ion evolution data, suggesting that the model used for the vacuum calculations is approximately correct but that the calculated gas load from outgassing in these regions was not large enough. This indicates that the design pressure in the EBIS will require the use of better materials in the ultrahigh vacuum chamber and the utilization of vacuum pumps with much higher pumping speeds.

The validity of the vacuum calculations is also verified by the experimental results obtained with the electron gun chamber, where the 
ultimate pressure was the same as the design pressure, $7.0 \times 10^{-10}$ Torr. The results obtained with the electron gun operations also show that the gas load from the electron gun is not appreciable after the cathode has been fully activated and that the vacuum model is correct in assuming that the electron gun chamber is essentially isolated from the ionization region. The ultimate pressure attained in the analysis system was only a factor of 3 greater than the calculated pressure, another indication of the validity of the vacuum calculations. Finally, although the working pressure in the extractor chamber increased more than one order of magnitude when the electron beam was turned on, the final working pressure was only 1.5 to 2.0 times higher than the ultimate pressure after the electron beam was focussed properly and operated for several hours. This was even true for continuous electron beam operation for the electron energies below $2.5 \mathrm{keV}$ used in these measurements, indicating that after the surfaces of the electron collector had been degassed by electron bombardment, the cooling carried away the power dissipated on it to prevent outgassing from elevated temperatures in this region.

The achievement of this low working pressure also indicates that the correct focussing conditions for the electron beam had been achieved. All the data collected on the electron gun and electron beam focussing system indicate that their performance was near the calculated values. Tr. a electron gun operater within the specifications given to the manufacturer, and a very stable electron beam was obtained over the entire operating range of the gun. The dispenser cathodes supplied with the electron gun were very durable under severe operating conditions, and the lifetime of the cathodes was more than a few thousand hours. In fact, the same cathode was used in the electron gun for all the measurements made on the ion source over a two year period, and it was reactivated many times after exposure to air or after gas poisoning caused by the electron beam being improperly focussed and striking the drift tubes or magnetic shim.

The experimental measurements incicate that the proper entrance conditions for the electron beam focussing system have been achieved. There are problems encountered in the extractor region, but the difficulty has 
been shown to be irelated to the focussing field of the solenoid, rather than the electron beam exir parameters. Local corrections to the magnetic field give better electron beam transmission and better ion extraction without any changes in the electron collectior-ion extractor geometry. Finally, the experimental data suggest that the proper focussing of the electron beam can be achieved in the presence of the trapping potentials and trapped ions, but the magnitude of the focussing field must be greater with these perturbations.

In fact, the results obtained with the test model EBIS give a clear indication of the techrical improvements that will make such an ion source a useful tool for production of highly-charged heavy ions. As already discussed, the most important of these are the achievement of a very low residual pressure and a very good focussing field. The first of these can be achicved, as suggested earlier, by the use of better ultrahigh vacuum materials in the system and by the use of more pumping in the ionization region where the residual vacuum must be the lowest. The liquid helium cryopumping surface originally built for the EBts prototype will certainly achieve these conditions, and it can also be used to improve the ionization of the working gas in another way. The helium cooled surface can be connected to the drift tubes on either side of the gas inlet drift tube with sapphire rods to cryopump any neutral atoms that flow into them. Then the injection of the jons into the electron beam can be controiled by the method of "electronic injection" (Donets and Pikin, 1974). Sapphire rodj could be used for this purpose because sapphire is a good thermal conductor and also a good electrical insulator at these temperatures. The working residual pressure would also be improved by the attainment of a uniform focussing field in the solenoid, since all the electrons lost to the drift tubes generate gases from them by their bombardment. The present results, as well as the results of other groups, indicate that the angular deviation of the magnetic axis should not exceed $10^{-3} \mathrm{mrad}$ and the radial field integral should not exceed 0.18 of the axial field for complete transmission of the electron beam and complete extraction of the ions through a cylindrical lens system. Such an accuracy has routinely been acnieved for the manufacturers of electron beam tubes, so a cryogenic solenoid is not the only solution 


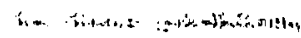

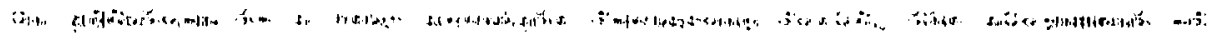

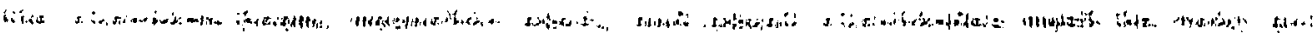

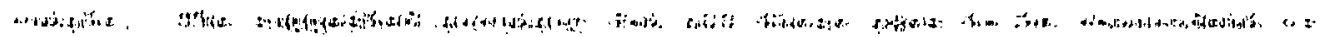
t.

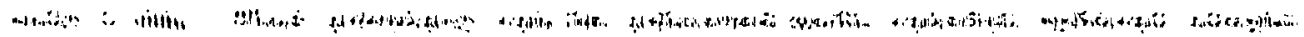

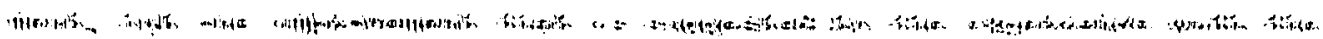

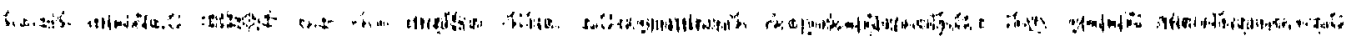

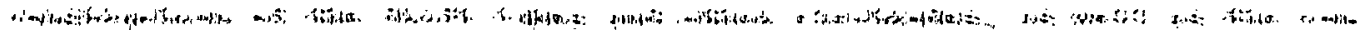
:-

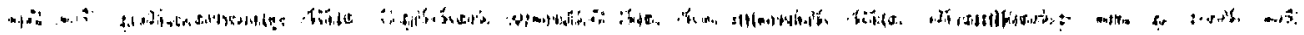

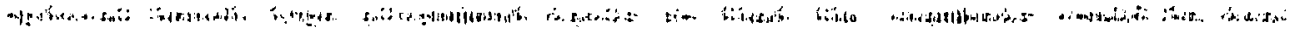

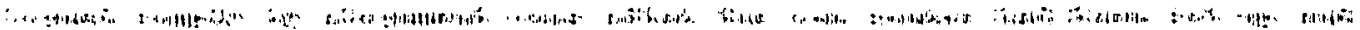

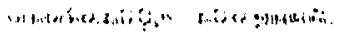

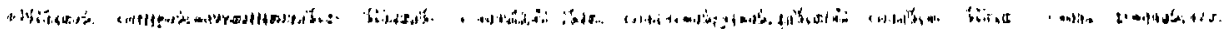

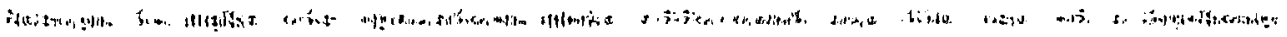

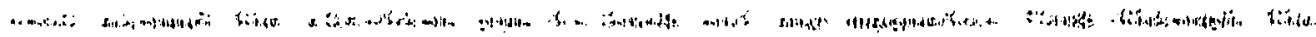

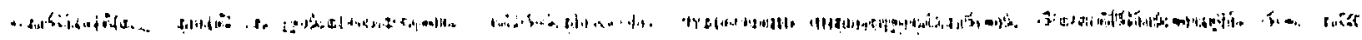

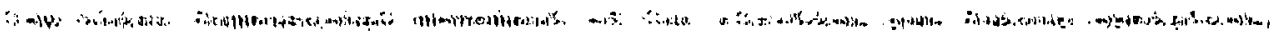

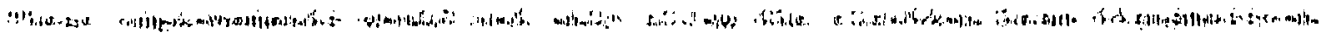

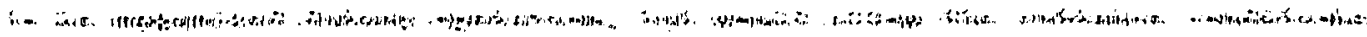

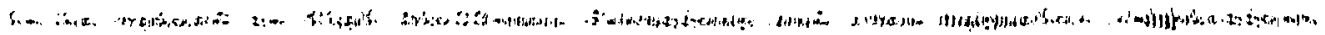

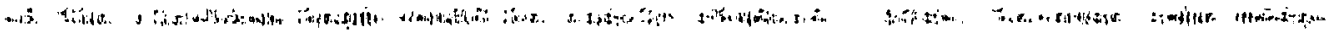

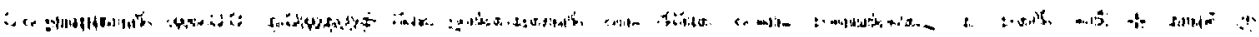

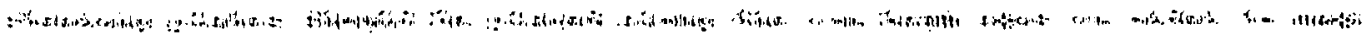

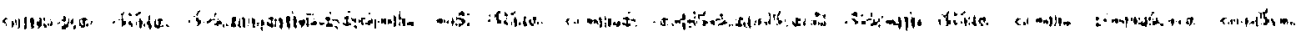

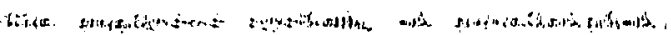

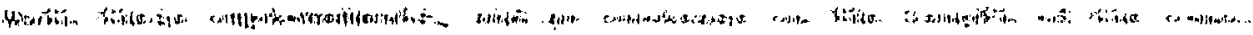

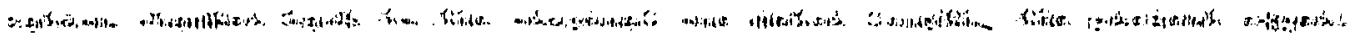

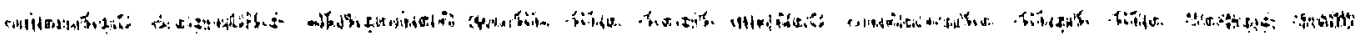

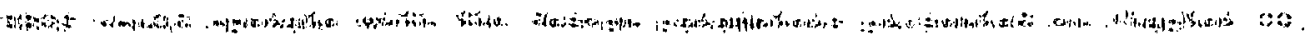

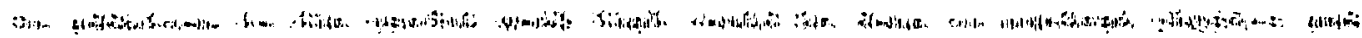

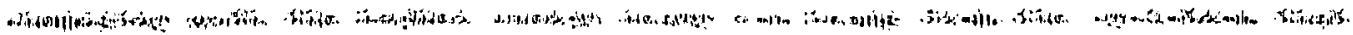

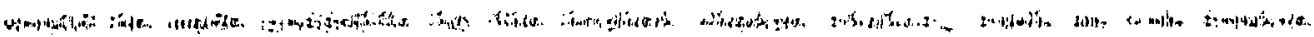

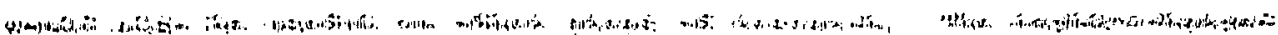




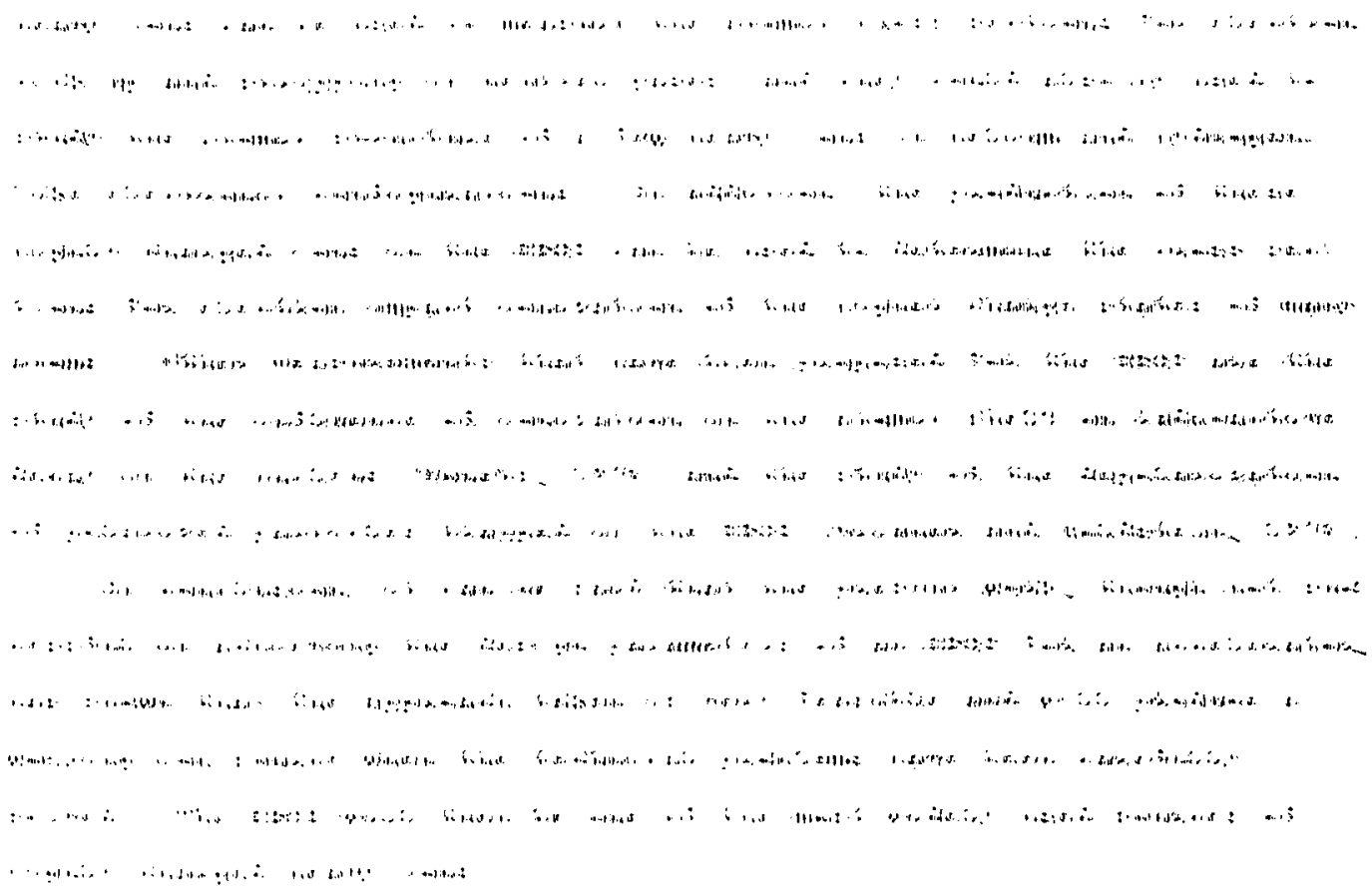




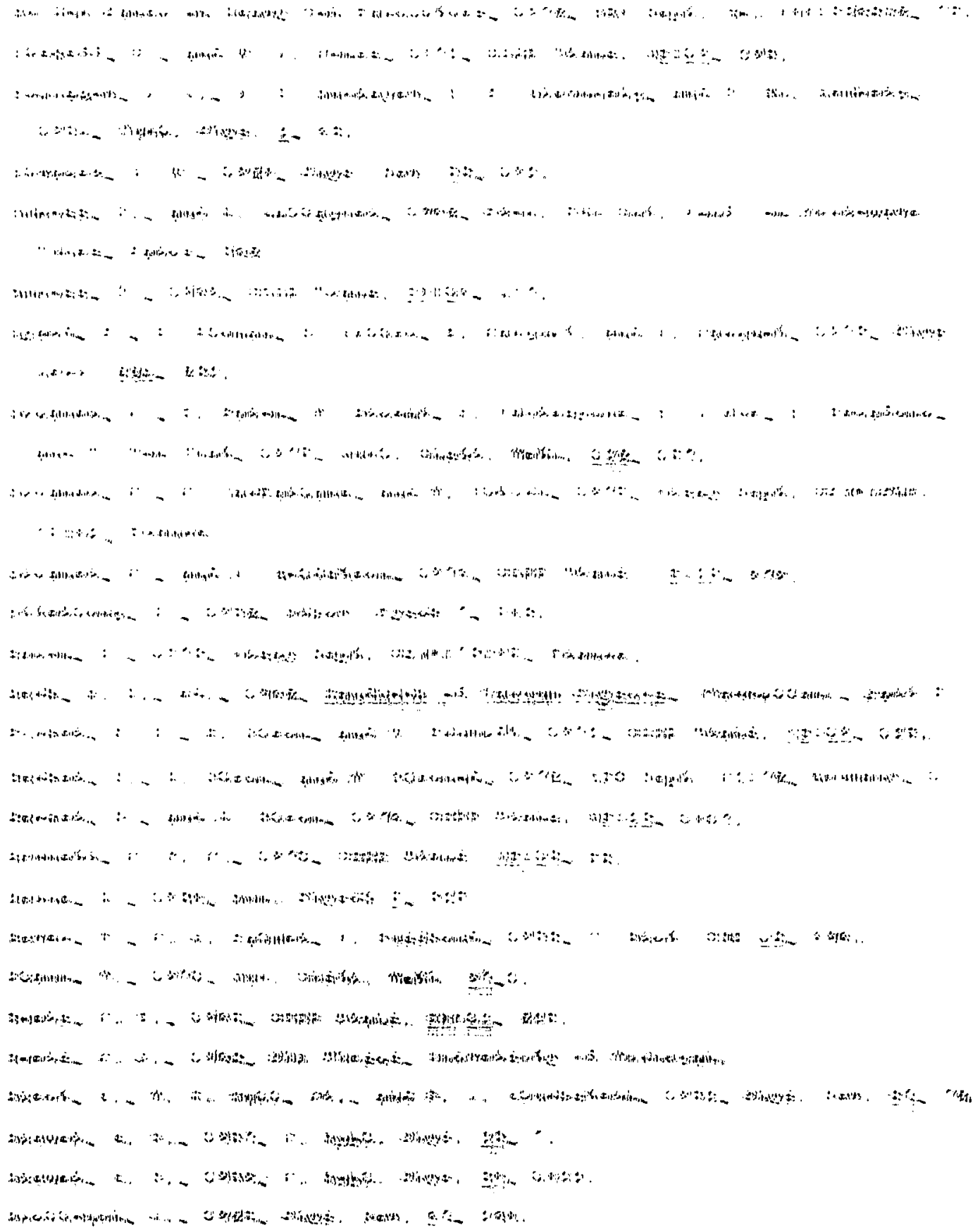




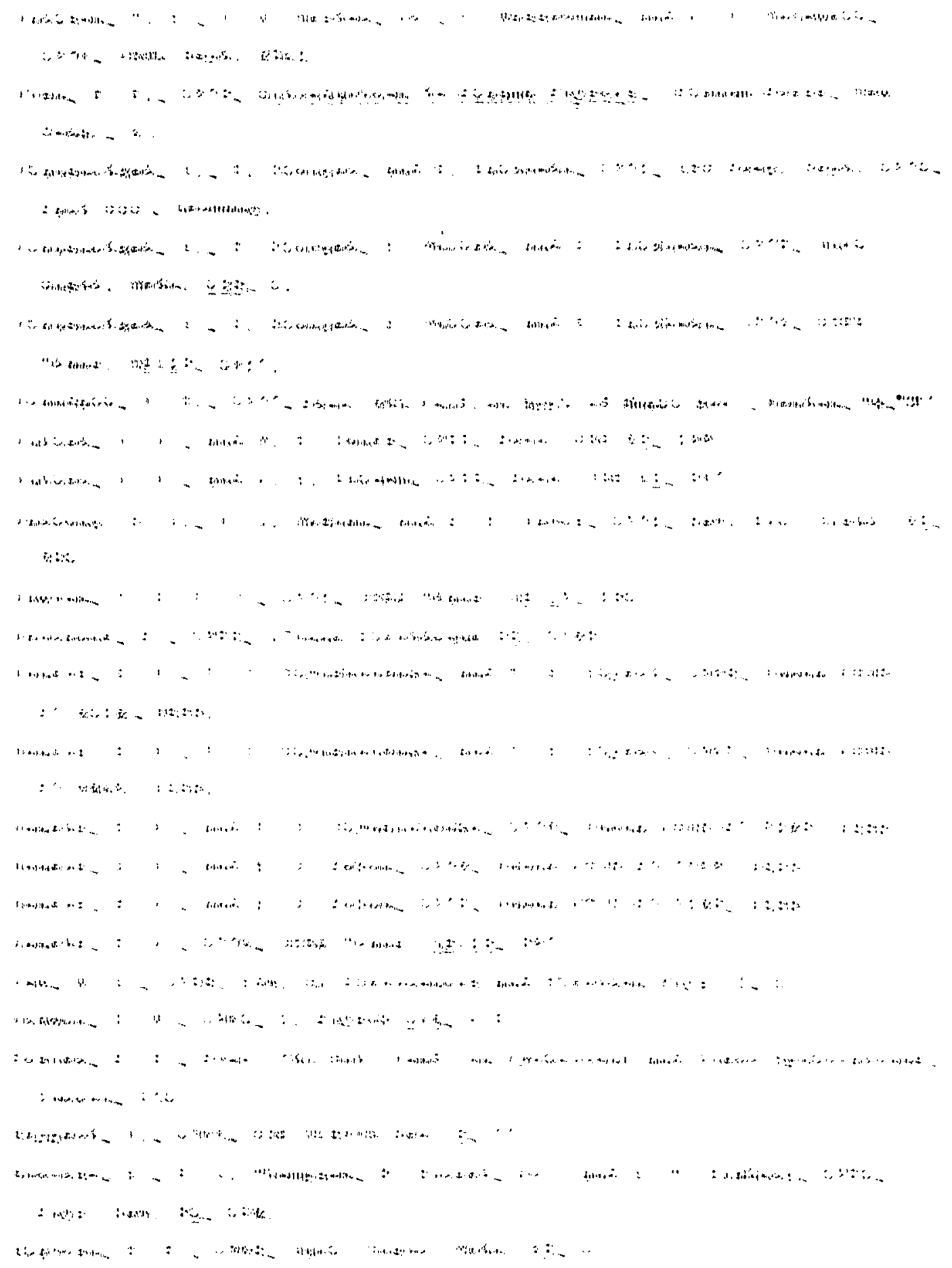




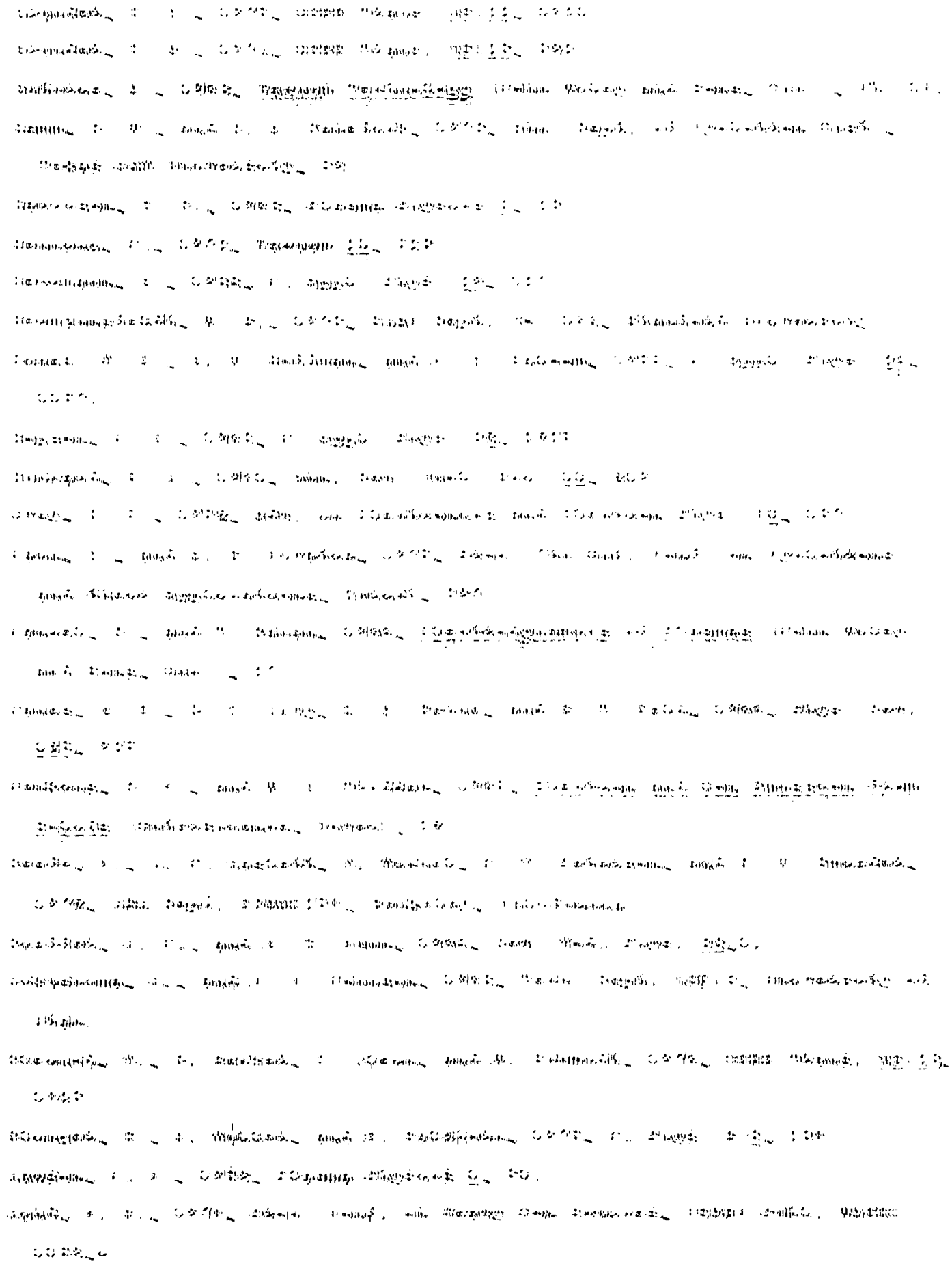




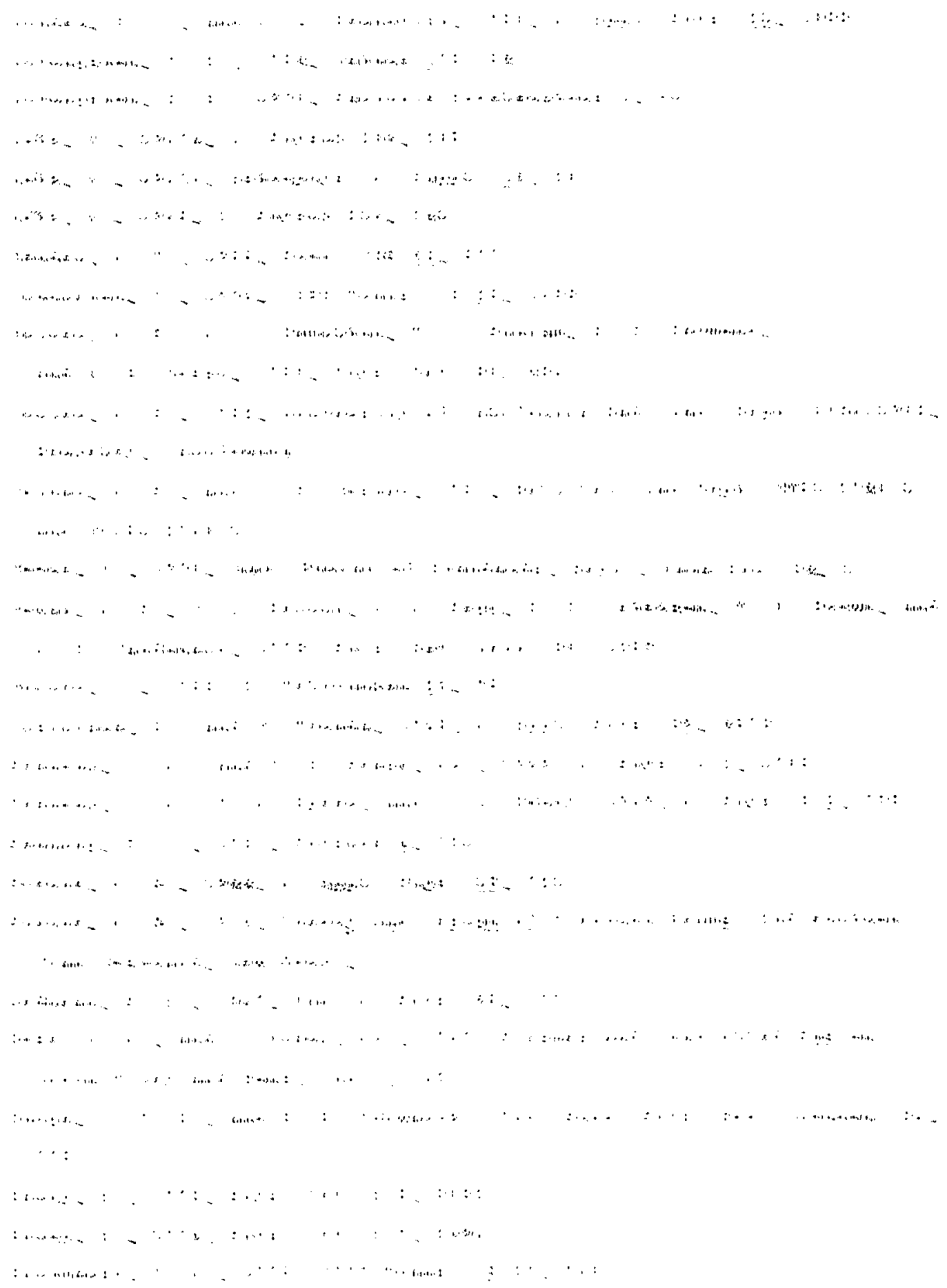




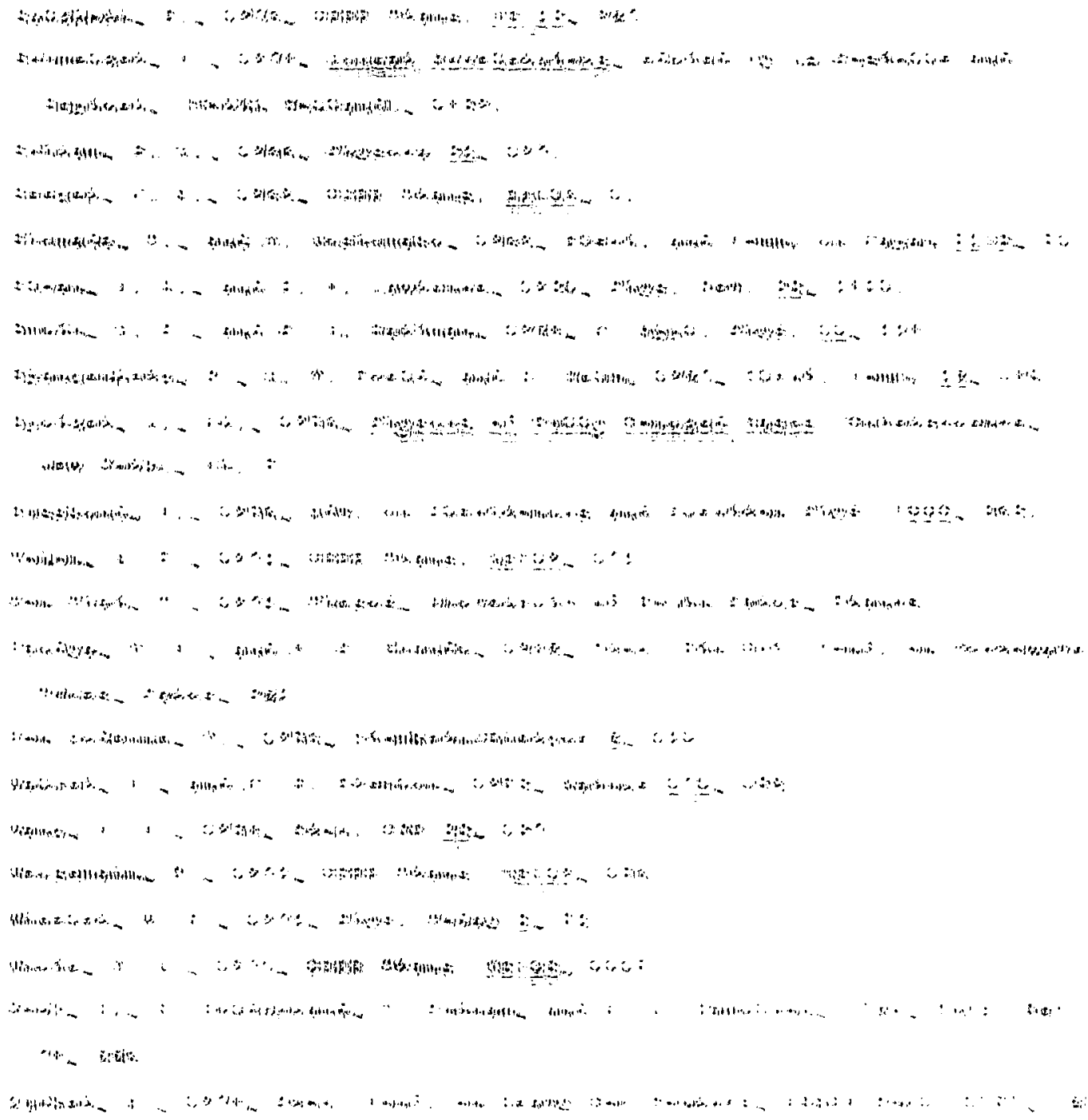

